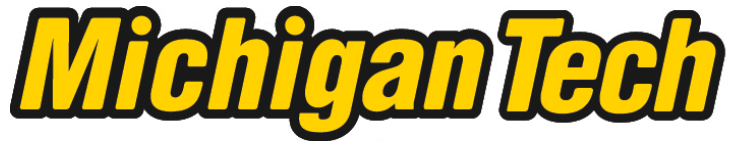 \\ Michigan Technological University Create the Future Digital Commons @ Michigan Tech
}

\section{Muffler characterization with implementation of the finite element method and experimental techniques}

Tyler W. Le Roy

Michigan Technological University

Follow this and additional works at: https://digitalcommons.mtu.edu/etds

Part of the Mechanical Engineering Commons

Copyright 2011 Tyler W. Le Roy

\section{Recommended Citation}

Le Roy, Tyler W., "Muffler characterization with implementation of the finite element method and experimental techniques", Master's Thesis, Michigan Technological University, 2011.

https://doi.org/10.37099/mtu.dc.etds/381

Follow this and additional works at: https://digitalcommons.mtu.edu/etds

Part of the Mechanical Engineering Commons 
MUFFLER CHARACTERIZATION WITH IMPLEMENTATION OF THE FINITE ELEMENT METHOD AND EXPERIMENTAL TECHNIQUES

\title{
By
}

Tyler W. Le Roy

\begin{abstract}
A THESIS
Submitted in partial fulfillment of the requirements for the degree of MASTER OF SCIENCE

(Mechanical Engineering)
\end{abstract}

MICHIGAN TECHNOLOGICAL UNIVERSITY

2011

(C) 2011 Tyler W. Le Roy 
This thesis, "Muffler Characterization with Implementation of the Finite Element Method and Experimental Techniques," is hereby approved in partial fulfillment of the requirements for the Degree of MASTER OF SCIENCE IN MECHANICAL ENGINERING.

Department of Mechanical Engineering-Engineering Mechanics

Signatures:

Thesis Advisor

Jason Blough

Department Chair

William Predebon

Date 


\section{Table of Contents}

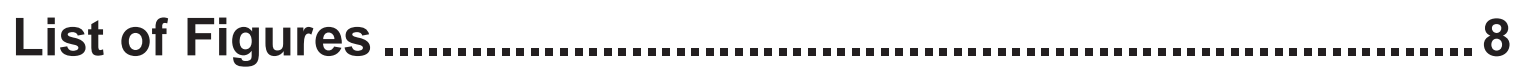

List of Tables........................................................................... 13

Acknowledgements ........................................................... 14

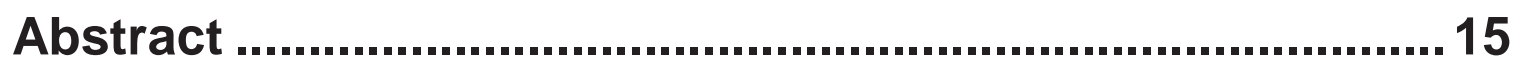

Chapter 1. Introduction............................................................ 16

1.1. Muffler Background ......................................................................17

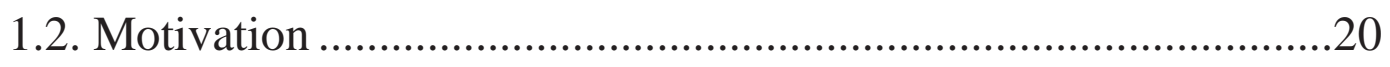

1.3. Review of Previous Research.........................................................22

Chapter 2. Theory of Muffler Acoustics ............................... 32

2.1. Theory of acoustic wave propagation ............................................32

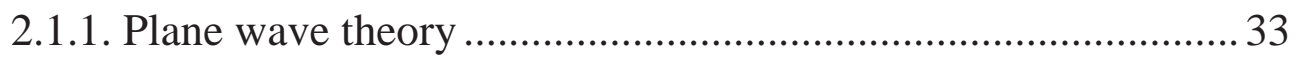

2.1.2. Three-Dimensional Wave propagation ..................................... 35

2.2. Transfer Matrix Method .............................................................. 38

2.3. Muffler Performance Parameters ..................................................43

2.3.1. Transmission Loss............................................................... 44

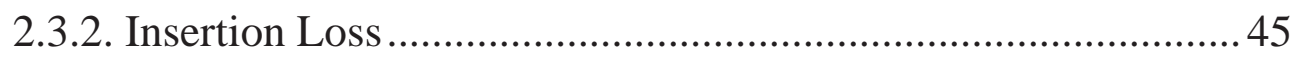

2.3.3. Level Difference ……………………………………………... 46

2.4. Theory of acoustic filters applied to mufflers ................................47

2.4.1. Uniform tube …………………………………………….... 47

2.4.2. Extended tube resonators ............................................................. 48 
2.4.3. Helmholtz and Concentric hole-cavity resonator 49

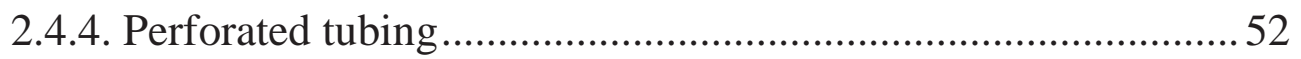

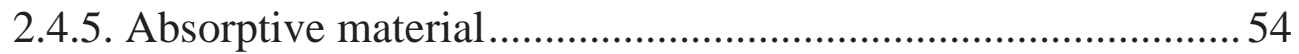

2.5. Additional effects on muffler performance..................................56

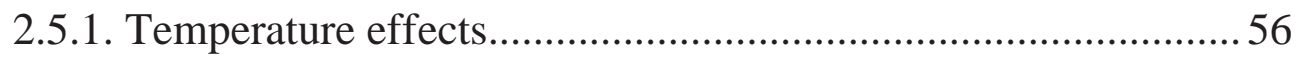

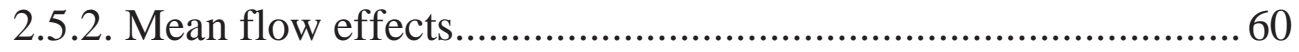

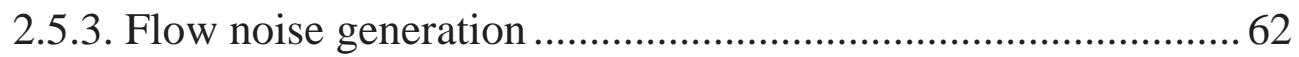

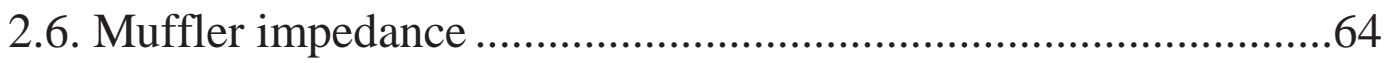

2.7. Implementation of Source Data .................................................65

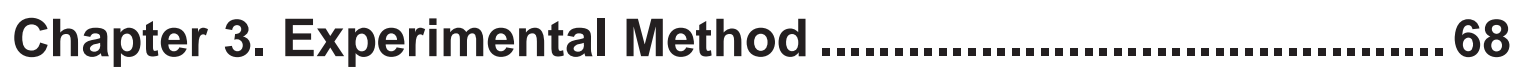

3.1. Transmission Loss measurement ..............................................68

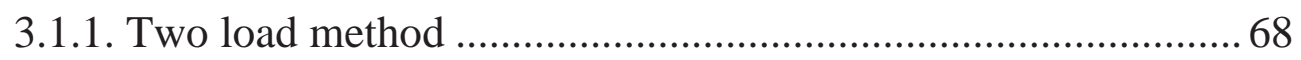

3.1.2. Two source - location method .................................................. 71

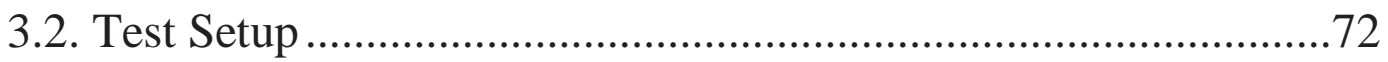

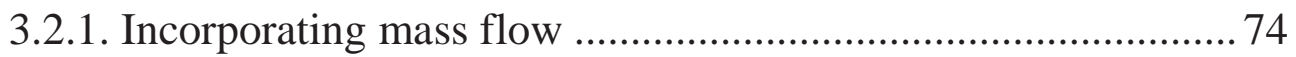

3.3. Equipment selection and signal processing................................76

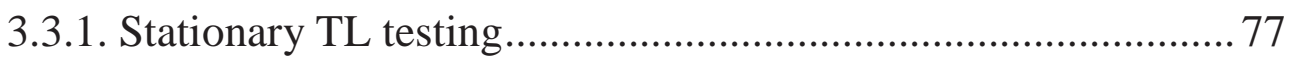

3.3.2. Mass flow TL testing ............................................................... 78

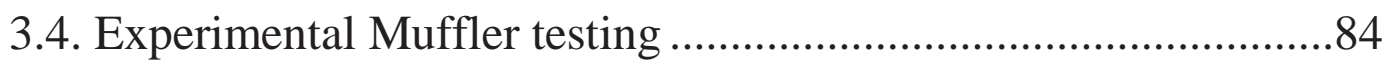

Chapter 4. Simulation Method .............................................. 89

4.1. Comparison of different muffler evaluation methods..................89

4.1.1. 1-D linear and non-linear techniques ........................................ 89

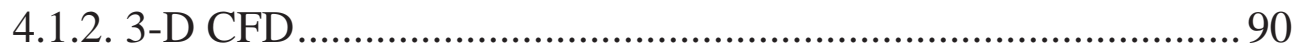

4.1.3. BEM and FEM method ......................................................... 91

4.2. Setting up an acoustic FEM model in VL ...................................99

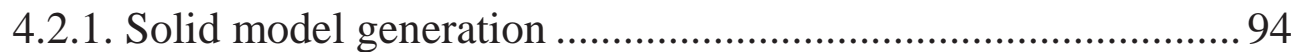


4.2.2. Mesh creation.... 95

4.2.3. Importing acoustic mesh and assigning properties ................... 96

4.2.4. Input and boundary conditions................................................ 98

4.2.5. Job creation and post processing ............................................... 98

4.2.6. SPL prediction using FEM....................................................... 100

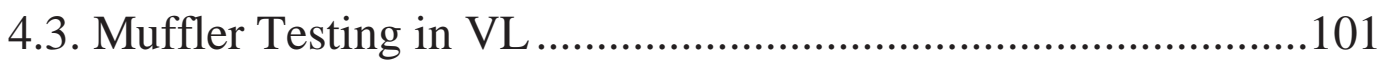

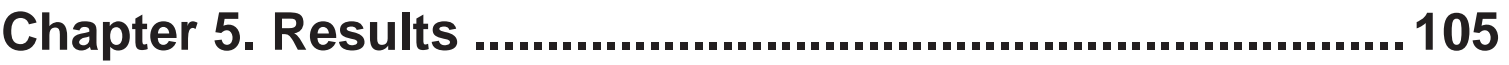

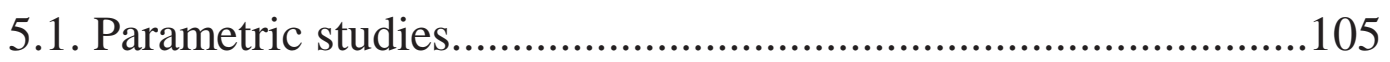

5.1.1. Case 1 - Change expansion tube diameter ............................... 105

5.1.2. Case 2 - Change the overall length of the empty expansion

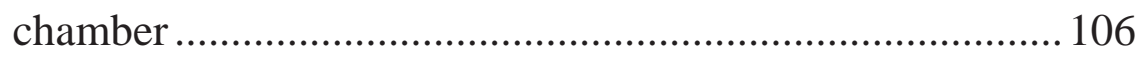

5.1.3. Case 3 - Change the length of the inlet and outlet tube with

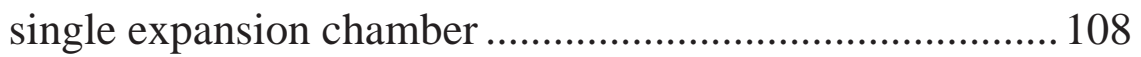

5.1.4. Case 4 - Change the diameter of the inlet and outlet tube with single expansion chamber ..................................................... 109

5.1.5. Case 5 - Addition of extended tubes in single expansion chamber.

5.1.6. Case 5.1 - Addition of perforated tubing for the single expansion chamber with 1 " extended tubes

5.1.7. Case 5.1.1 - Modifying the perforation properties of single expansion chamber with 1" perforated and 1" extended tubing

5.1.8. Case 6 - Addition of baffles inside of the single expansion chamber

5.1.9. Case 7 - Addition of extended tubes to dual expansion chamber 
5.1.10. Case 7.1 - Addition of perforated tubing for the dual expansion chamber with 1" extended tubes ............................................ 118

5.1.11. Case 7.1.1 - Modifying the perforation properties of single expansion chamber with 1" perforated and 1" extended tubing

5.1.12. Case 8 - Absorptive material is added to the dual expansion chamber with 1" extended tubes.

5.1.13. Case 8.1 - Modifying the absorptive material properties for $1 / 2$ " material dual expansion chamber with 1 " extended tubes

5.1.14. Case 9 - Addition of mean flow through empty single expansion chamber

5.1.15. Case 10 - Addition of mean flow through dual expansion chamber with 1" extended tubes

5.1.16. Case 11 - Temperature effects on dual expansion chamber with 1 " extended tubes 128

5.2. Correlated results 129

5.2.1. Correlation 1 - Eight inch diameter empty muffler 129

5.2.2. Correlation 5 - Extended tubes added to eight inch diameter muffler

5.2.3. Correlation 5.1 - 1inch perforated tubing with 1inch extended tubes added to the single expansion chamber

5.2.4. Correlation 6 - Dual expansion chamber muffler 132

5.2.5. Correlation 7 - 1" extended tubes added to dual expansion chamber

5.2.6. Correlation 7.1 - 1" perforated tubing with 1" extended tubes added to dual expansion chamber 
5.2.7. Correlation 8 - Absorptive material with thicknesses of 0.25 " and 0.5 " added to dual expansion chamber with 1 " extended tubes 135

5.3. Transmission Loss with mass flow rate ...................................136

5.4. Flow generated noise levels ...................................................139

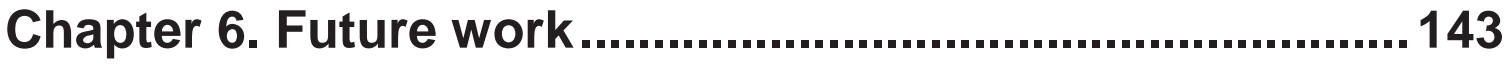

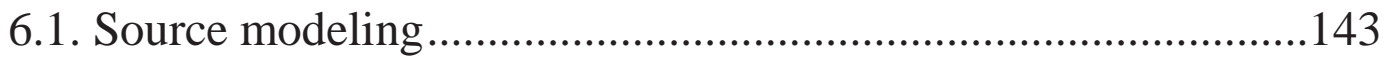

6.1.1. Predicting sound pressure levels ................................................ 143

6.2. Incorporating temperature effects in experimental setup...........144

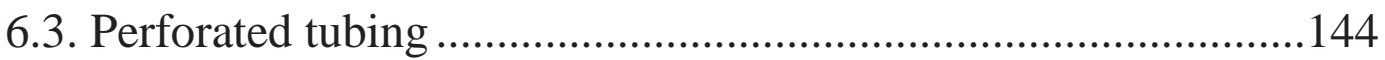

6.4. Increasing capability of TL measurement with flow .................144

Chapter 7. Conclusion ......................................................... 146

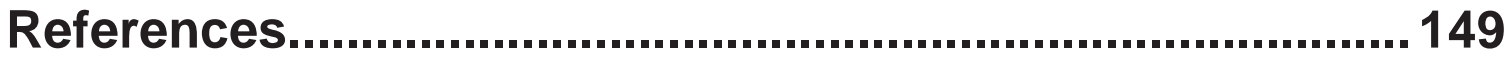

Appendices.....................................................................153

7.1. Appendix A: Additional Figures from FEM Study ...................153

7.2. Appendix B: Perforated Tubing MatLab Script.........................158 


\section{List of Figures}

Figure 1.1: Typical first order firing frequencies for various engine configurations. ...... 18

Figure 1.2: Diagram of original muffler test setup for determining Transmission Loss

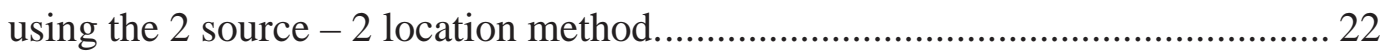

Figure 2.1: The cut-on frequency of higher order mode effects in a cylindrical muffler as a function of diameter.

Figure 2.2: (a) Plane wave propagation at $400 \mathrm{~Hz}$ and (b) 3-D wave propagation at $1800 \mathrm{~Hz}$ in an empty eight inch diameter chamber. 38

Figure 2.3: Location of the state vectors $p, v$ for the two element acoustic filter..... 40

Figure 2.4: The three basic types of elements in an exhaust system using the transfer matrix method.

Figure 2.5: Acoustical Transmission network of an entire exhaust system including acoustical source and termination where networking for the filter is not depicted.

Figure 2.6: Acoustical Transmission network for the three different elements used in acoustic filters.

Figure 2.7: Quarter wave resonator diagrams for (a) extended inlet/outlet and (b) flow reversal (expansion/contraction)

Figure 2.8: Diagram of a Helmholtz resonator. 50

Figure 2.9: Transmission Loss of a simple Helmholtz resonator. ................................. 51

Figure 2.10: Diagram of a concentric tube resonator. ................................................. 52

Figure 2.11: Transmission Loss of a cylindrical muffler when the temperature of the medium is varied.

Figure 2.12: One port model to determine source properties.

Figure 3.1: Two Load method in determining TL of muffler for (a) step 1 when outlet is open to atmosphere and (b) step 2 when load at outlet is rigidly closed.

Figure 3.2: Two Source-Location method in determining TL of muffler for (a) step 1 when source is at inlet and (b) step 2 when source is at outlet. 
Figure 3.3: The developed flow bench showing (a) the flow meter and tube extension and (b) the air velocity source (Shop Vacuum) and controller (variable autotransformer)

Figure 3.4: Flow noise spectrums inside of the TL test tube at microphone 3 (reference channel). The speed of sound was taken as $348 \mathrm{~m} / \mathrm{s}$. 80

Figure 3.5: The flow bench test configuration for Transmission Loss testing. 81

Figure 3.6: A comparison of different FRF measurement methods compared to the noise generated from turbulent flow. 83

Figure 3.7: The overall dimensions of the baseline muffler used while completing this thesis.

Figure 3.8: A picture showing the extended tubes and perforated tubing that was used during the completion of this thesis. 86

Figure 3.9: A picture showing the internal baffle, extended tubes, and perforated tubing used for one of the correlated experimental models. 87

Figure 3.10: Two layers of absorptive material installed in the muffler. ........................ 88

Figure 4.1: The CAD model for one of the mufflers used in this thesis......................... 94

Figure 4.2: A mesh created for a dual expansion chamber with extended tubes and perforations using $1 / 4$ " elements 95

Figure 5.1: Case 1 parametric study - the diameter of the expansion tube was modified for the single expansion chamber. 106

Figure 5.2: Case 2 parametric study -The length of the expansion chamber is varied for the single expansion chamber.

Figure 5.3: Case 3 parametric study -the length of the inlet and outlet tube are varied for the single expansion chamber. 108

Figure 5.4: Case 4 parametric study - the diameter of the inlet and outlet tube are varied for the single expansion chamber. 109

Figure 5.5: Case 5 parametric study - extended tubes are added to the single expansion chamber. 111

Figure 5.6: Case 5.1 parametric study - perforated tubing is added to the single expansion chamber with 1" extended tubes. 
Figure 5.7: Case 5.1.1 parametric study - The perforated tubing properties were modified for the single expansion chamber with 1" perforated and 1" extended tubes..... 113

Figure 5.8: The model for the three chamber muffler with 0.5 " partitions. 114

Figure 5.9: Case 6 parametric study - The single expansion chamber is split into equal length chambers where the baffle is modeled as infinitely thin. 115

Figure 5.10: Case 6 parametric study - The single expansion chamber is split into equal length chambers where the baffle is modeled as 0.5 " thick 116

Figure 5.11: Case 7 parametric study - extended tubes are added to the dual expansion chamber where the baffle is modeled as infinitely thin.

Figure 5.12: Case 7 parametric study - extended tubes are added to the dual expansion chamber where the baffle is modeled as 0.5 " thick. 118

Figure 5.13: Case 7.1 parametric study -perforated tubing is added to the dual expansion chamber with 1" extended tubes. The baffle is modeled as infinitely thin. 119

Figure 5.14: Case 7.1 parametric study -perforated tubing is added to the dual expansion chamber with 1 " extended tubes. The baffle is modeled as 0.5 " thick. 120

Figure 5.15: Case 7.1.1 parametric study - the perforation properties are modified for the dual expansion chamber with 1" perforated and 1" extended tubes. The baffle is modeled as infinitely thin. 121

Figure 5.16: Case 7.1.1 parametric study - the perforation properties are modified for the dual expansion chamber with 1" perforated and 1" extended tubes. The baffle is modeled as $0.5 "$ thick.

Figure 5.17: The model for the two chamber muffler with 1/2" material and 1" extended tubes. 122

Figure 5.18: Case 8 parametric study - absorptive material is added to the dual expansion chamber with 1" extended tubes. The baffle is modeled as infinitely thin

Figure 5.19: Case 8 parametric study - absorptive material is added to the dual expansion chamber with 1 " extended tubes. The baffle is modeled as 0.5 " thick. 123

Figure 5.20: Case 8.1 parametric study - absorptive material properties are modified for the 1" material dual expansion chamber with 1" extended tubes. The baffle is modeled as infinitely thin 
Figure 5.21: Case 8.1 parametric study - absorptive material properties are modified for the 1" material dual expansion chamber with 1" extended tubes. The baffle is modeled as $0.5 "$ thick.

Figure 5.22: Case 9 parametric study - mean flow is incorporated into the single expansion chamber model.

Figure 5.23: Case 10 parametric study - mean flow is incorporated into the dual expansion chamber model. The baffle is modeled as infinitely thin.

Figure 5.24: Case 11 parametric study - temperature effects are applied to the dual expansion chamber model. The baffle is modeled as infinitely thin. 128

Figure 5.25: Case 1 correlation study - Eight inch diameter empty muffler..... 129

Figure 5.26: Case 5 correlation study - 1" and 2" extended tubes added to eight inch diameter muffler.

Figure 5.27: Case 5.1 correlation study - 1" perforated and 1" extended tubes in single expansion chamber..

Figure 5.28: Case 6 correlation study - dual expansion chamber muffler 132

Figure 5.29: Case 7 correlation study - 1" extended tubes are added to the dual expansion chamber muffler.

Figure 5.30: Case 7.1 correlation study - 1" perforated tubing with 1" extended tubes added to dual expansion chamber

Figure 5.31: Case 8 correlation study - Absorptive material added to dual expansion chamber with 1" extended tubes 135

Figure 5.32: TL results for a 6" diameter empty muffler with mass flow. 137

Figure 5.33: TL results for a 6" diameter muffler with 1" perforated tubing which incorporated mass flow. 138

Figure 5.34: Flow noise generated inside of the mufflers when the source is used as a blower and applied a velocity of $47 \mathrm{~m} / \mathrm{s}$ to the inlet.

Figure 5.35: Flow noise generated inside of the mufflers when the source is used as a blower and applied a velocity of $47 \mathrm{~m} / \mathrm{s}$ to the inlet.

Figure 5.36: The overall sound pressure level as a function of the flow speed for the 6" Diameter empty muffler. 142 
Figure A.1: FEM results for a few single expansion chamber mufflers....................... 154

Figure A.2: Experimental results for a few single expansion chamber mufflers............ 155

Figure A.3: FEM results for a few dual expansion chamber mufflers.......................... 156

Figure A.4: FEM results for a few dual expansion chamber mufflers......................... 157 


\section{List of Tables}

Table 2.1 The comparison of an acoustical transmission network with the corresponding

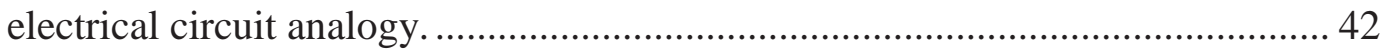

Table 2.2 Sample Helmholtz resonator properties used for calculation of Transmission

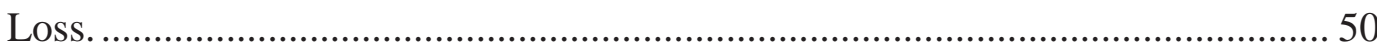

Table 3.1 The frequencies $(\mathrm{Hz})$ when the transmission loss calculation breaks down when $c=348 m s$. 74

Table 4.1 A description of the parametric studies completed throughout the duration of this thesis 101

Table 4.2 Perforated tubing properties for specimens used in study. 103

Table 5.1 The absorptive materials properties assigned to models for Case 8.1 124 


\section{Acknowledgements}

I would like to thank my advisor, Dr. Jason Blough, for influencing me to complete my Master's. You have made that decision an easy and enjoyable one. I would also like to thank Jason for his financial and technical support; I couldn't have completed this thesis without your guidance. I would also like to thank the other DSP faculty, Dr. Chuck Van Karsen and Dr. James De Clerk. Both of you have greatly influenced my passion in NVH and have shaped my career path moving forward. I would like to thank Raghavan Vasudevan, from LMS, for your technical support in FEM and Virtual.Lab. Also, I would like to thank my committee member, Chris Plumber, for your time and commitment.

I would also like to thank my parents, John and Kris Le Roy. You both have always believed in me and have stood by me as I have pursued my passions. Both of your supports have allowed me to become the person I am today. Thank you for everything. Lastly, but not least, I would like to thank Elizabeth. Elizabeth, you have always been by me and have pushed me to be my very best. I appreciate the numerous hours you put in helping me build the mufflers, run the tests, and editing every word of this thesis. I couldn't have done it without you! 


\section{Abstract}

Determining how an exhaust system will perform acoustically before a prototype muffler is built can save the designer both a substantial amount of time and resources. In order to effectively use the simulation tools available it is important to understand what is the most effective tool for the intended purpose of analysis as well as how typical elements in an exhaust system affect muffler performance. An in-depth look at the available tools and their most beneficial uses are presented in this thesis. A full parametric study was conducted using the FEM method for typical muffler elements which was also correlated to experimental results.

This thesis lays out the overall ground work on how to accurately predict sound pressure levels in the free field for an exhaust system with the engine properties included. The accuracy of the model is heavily dependent on the correct temperature profile of the model in addition to the accuracy of the source properties. These factors will be discussed in detail and methods for determining them will be presented.

The secondary effects of mean flow, which affects both the acoustical wave propagation and the flow noise generation, will be discussed. Effective ways for predicting these secondary effects will be described. Experimental models will be tested on a flow rig that showcases these phenomena. 


\section{Chapter 1. Introduction}

Emission requirements have exponentially become much more stringent on engine noise and emission levels over the last decade and a half. A major factor in engine and vehicle emitted noise is the exhaust system. With regard to the sound pressure levels, the untreated exhaust noise is often ten times greater than all the structural noise of the system combined (1). Thus, there has been a great deal of research and development in the area of predicting muffler performance.

Until recently with the advancement of the computer age there was a great deal of limitation for predicting muffler performance. In the past, the mufflers often needed to be scaled down to a simplified model which left out some key factors that contributed to the overall sound pressure level. Although the underlying theory was in place due to M. L. Munjal's classical book on duct acoustics (2), the tools and resources to fully characterize the entire system were not available until computer solvers for Finite Element Methods (FEM) and Computational Fluid Dynamics (CFD) became readily available.

The methods in place to predict muffler performance never took the entire picture into account. Effects on muffler performance such as flow induced noise, temperature gradients, back pressure, shell break-out noise, muffler and source impedances, and many others have been relatively unexplored until recently. The researcher hopes to address these additional parameters as well as discuss the benefits and limitations of the current techniques of muffler characterization within this thesis. 


\subsection{Muffler Background}

Typically mufflers are classified under two different categories, dissipative and reflective. A dissipative muffler is based on the principle of converting the exhaust noise energy caused by fluctuating pressure waves into heat. This is done by using acoustic materials such as porous sound attenuating woven fibers and perforated tubing. The woven fibers have been enhanced over the years to withstand high temperatures often in excess of $500^{\circ} \mathrm{C}$ and provide good attenuation across the mid to high frequency ranges (3). These materials have become much more durable and their use in hazardous conditions (such as in exhaust systems) has become much more common. Another benefit of dissipative mufflers is that the pressure drop across the system is relatively low because the flow path is not significantly altered by flow reversals, twists and turns, or by any other means. The downside of dissipative mufflers is that they are generally insufficient in the low frequency range as the wavelength is much too large to be attenuated by the material (2). The finite thickness of the material causes a decrease in expansion of the sound pressure waves in a cavity. As a result, the material will actually decrease the muffler performance at low frequencies. Therefore, there is an absolute thickness of absorptive material which is ideal for every scenario.

In determining the lowest frequencies of interest, it is essential to understand the application of the exhaust system. The primary parameter of interest is the firing frequency of the engine which is the rate of in-cylinder fuel ignition which takes all active cylinders into account (4). The calculation for firing frequency is shown in Equation 1-1. Other frequencies expected in the spectrum are harmonic orders of both the firing frequency and crankshaft speed, usually with diminishing amplitudes as a function of frequency. In the application of an exhaust system for a mid to small sized vehicle, the first primary firing frequency 
is often too low to be attenuated by the absorptive material. Typical firing frequencies, $F_{F}$, for various types of engines are shown in Figure 1.1. In the automobile industry a common firing frequency is below 150 to $200 \mathrm{~Hz}$, a dissipative muffler would be insufficient without an additional method for attenuating the first firing frequency.

$$
F_{F}(H z)=\frac{R P M}{60} \cdot \frac{N}{S}
$$

Equation 1-1

where:

$$
\begin{gathered}
R P M=\text { Crankshaft revolution speed }\left(\frac{\text { rev }}{\min }\right) \\
N=\text { number of active cylinders } \\
S=2(4-\text { stroke }), 1(2-\text { stroke })
\end{gathered}
$$

The second muffler classification is the reflective muffler. This type of muffler uses the geometry of the complex muffler components to create impedance mismatches with the incoming exhaust stream in an attempt to cancel

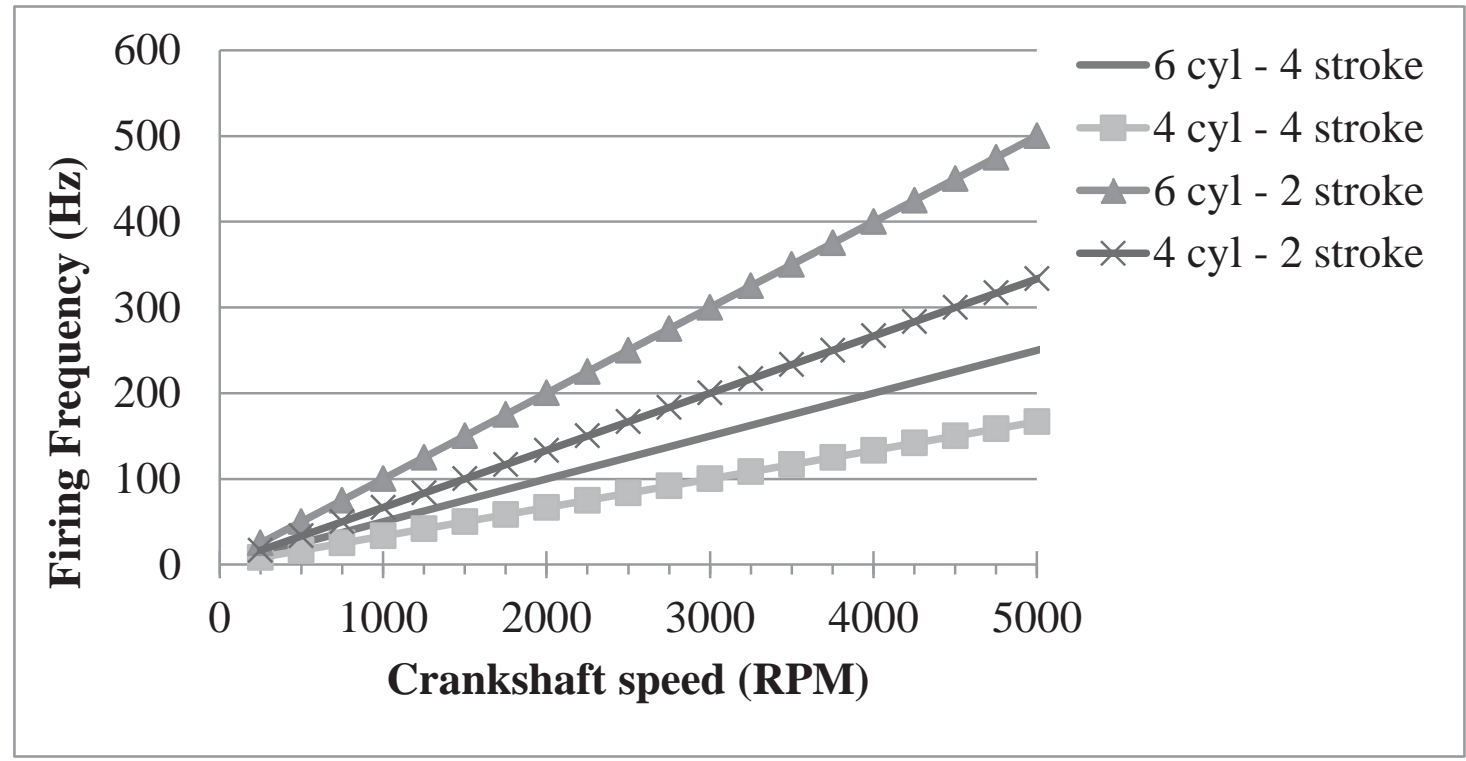

Figure 1.1: Typical first order firing frequencies for various engine configurations. 
out the progressive pressure wave (5). Typically, these mufflers consist of multiple chambers, flow reversals, and end resonators. The benefit of a reflective muffler is that it has the potential to work well at low frequencies where the first firing order of the engine might occur. Another asset is that these units are relatively cheap to build, both as a manufacturing and materials standpoint. Theoretically, elements in a reflective muffler could be tuned to a frequency where there is an acoustical resonance in the system. An acoustical resonance will cause all the exhaust energy to be absorbed at a particular frequency. This could be particularly important when the operating condition or load is unchanging (such as in a generator). Although designing for a resonance at such frequencies is impractical when there are fluctuating variables such as temperature gradients and load conditions. The downside of this form of muffler is that there are areas in the frequency range of interest where there is little attenuation of the exhaust sound pressure (5). Also, after the cutoff frequency when three dimensional sound waves start to propagate, the performance of the muffler significantly deteriorates.

In practice however, most production mufflers consist of a combination of reflective and dissipative mufflers. Thus, the benefits of both types of elements are seen. The reflective elements typically target the first few orders of the firing frequency whereas the dissipative elements attenuate most of the mid to high orders of firing (starting around $300-400 \mathrm{~Hz}$ ) along with the flow induced noise caused by the turbulent flow through the exhaust system.

A few of the limiting factors that often drive the design of the muffler are cost, packaging, and manufacturing concerns. On average, mufflers that contain sound absorptive materials cost substantially more which can limit their use.

Although in the past twenty years they have been used more frequently as a result of a drop in cost and increased durability. In most applications, such as automotive, the packaging constraints are fixed to a specific size. Because there is 
not a lot of room to expand the muffler chamber, it is crucial that the given space requirements work as effectively as possible. Other important factors are the overall feasibility of the design and cost of machining the parts that are within the muffler.

\subsection{Motivation}

Muffler development has often been done by trial and error or on previous analogies of an electrical circuit which will be discussed in later chapters. This has been shown to be very ineffective and time consuming, thus numerical techniques are being integrated into the design phase. The numerical analysis is typically formulated on the idealized case where mass flow, temperature gradients, and source characteristics are not taken into account. Although these types of numerical calculations may serve as a decent precursor to how the muffler will perform, they often can be misleading if interpreted directly. In order to fully utilize the tools available, these factors need to be addressed from a simulation and experimental stand point.

Backpressure has also been a cause for concern. The two most commonly used methods, the FEM and the BEM method, cannot solve for static pressures. This causes designers to rely on 1-D simulation (such as GT-Power) or CFD to determine backpressure. Each method lies at complete opposite ends of the complexity spectrum. Whereas CFD is often too computationally expensive and time consuming to conduct, 1-D simulations are considered too simplistic and the accuracy tends to be questionable at best.

This thesis uses the most common numerical techniques for both the FEM and BEM method as well as experimental techniques to correlate the simulation 
results and to confirm the accuracy of the models. Models were built to predict muffler performance for the most common reflective and absorptive type elements. Parameters were varied to understand the effect of the elements on muffler performance. Other properties that were varied in the numerical models include mass flow, temperature gradients, absorptive material properties, and perforated tubing properties. Acoustic modeling of the source will be explained and steps will be described to accurately predict sound pressure levels (SPL) using FEM. Source modeling was performed to predict SPLs on a confidential project. Due to the limited source data on another system, only the theory and specific modeling instructions are presented. All other numerical techniques including CFD, 1-D simulations, and Hybrid-FEM will be examined.

Experimental testing was conducted for each different property addressed above until there was a high level of certainty that the numerical trend patterns agree with the experimental results. Differences between the experimental and numerical results will be addressed and the corresponding limitations concluded upon. In order to incorporate mass flow, a flow bench needed to be developed and implemented into the current setup that was built by Justin Keske during the course of his PhD studies at Michigan Tech (6). A diagram of the original test setup is shown in Figure 1.2. The new test setup with the flow bench installed will be shown in Chapter 5. The flow bench was used during the course of this research project to understand the effects of mass flow rate on muffler performance. Also, flow induced noise levels at various flow speeds were measured for a few different muffler configurations. The results from the flow bench were determined using a constant volume velocity source applied to the inlet. In operation on an engine there would be a fluctuating velocity source. 


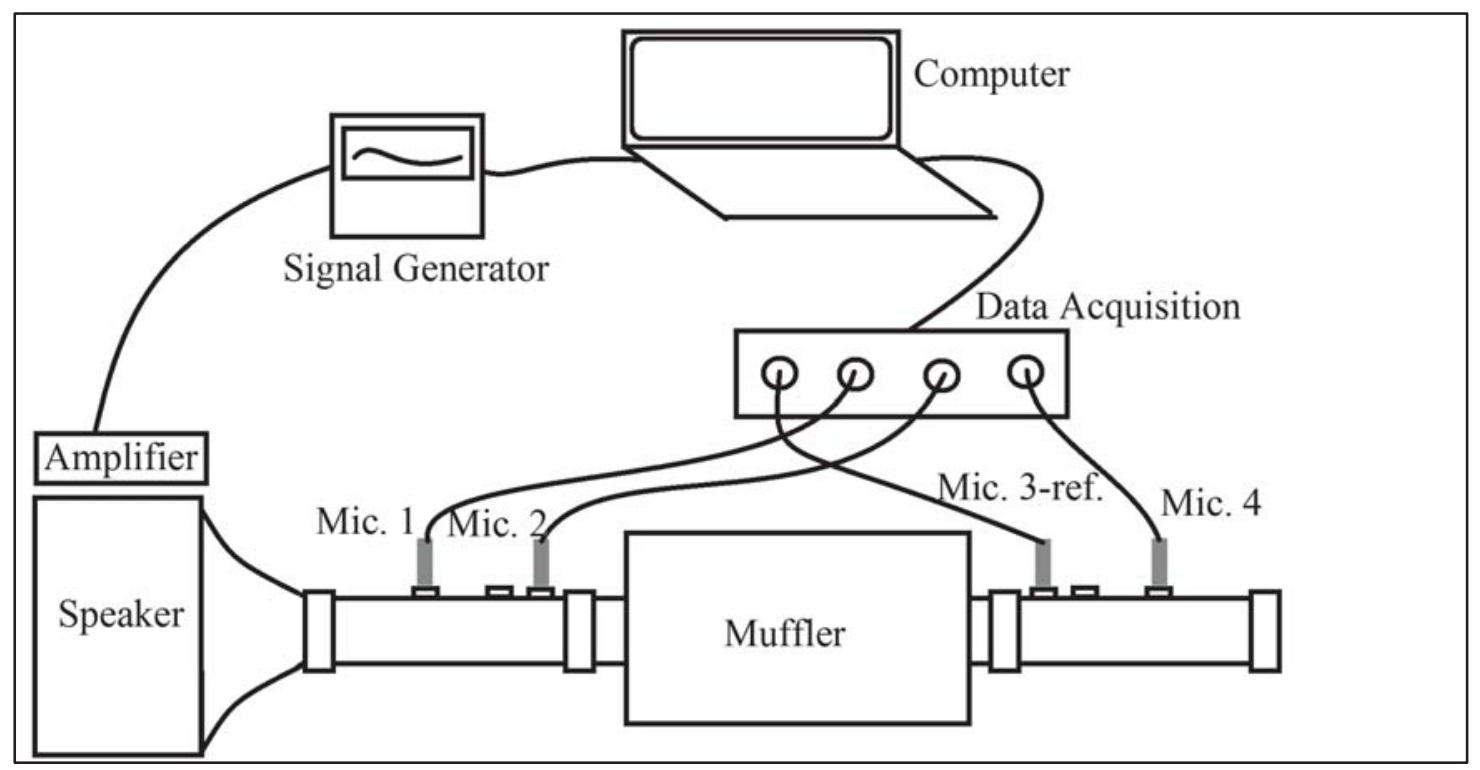

Figure 1.2: Diagram of original muffler test setup for determining Transmission Loss using the 2 source -2 location method.

After reading this thesis, the reader will have a firm understanding of current practices and research areas in muffler development along with the limitation and benefits of each. The reader will also be familiar with the key variables not currently implemented in commercial codes which is where future research will be heavily needed. The topics addressed within this thesis are relatively unexplored. The idea of the author was to examine many of the unknown variables rather than to concentrate on a specific topic in muffler performance. Because of this, there is a potential for many research investigations to stem from this thesis.

\subsection{Review of Previous Research}

This section covers the current research topics in muffler development. To become familiar with the context of this section an introduction to muffler 
development needs to be defined. The underlying theory and past investigations will be covered in Chapter 2.

Muffler performance from a designer's standpoint is characterized by either the Transmission Loss (TL) or Insertion Loss (IL) of the muffler. Transmission Loss is the ratio of the sound power of the incident (progressive) pressure wave at the inlet of the muffler to the sound power of the transmitted pressure wave at the outlet of the muffler (2). The benefit of TL is that it is a parameter of the muffler alone and the source or termination properties are not needed. Because of the simplifications, the TL is the most common and versatile parameter for muffler performance. Insertion loss is defined as the difference between the acoustic pressure radiated from the source with and without the muffler attached (2). Insertion Loss is a more accurate method of determining muffler performance as it is measured in actual operating conditions with the source properties applied.

Until recently, most of the research on mufflers was based upon accurately predicting the transmission loss of muffler features using both numerical and analytical techniques. Many parametric studies were done mainly through the works of Selamet et al. and Munjal to better understand and predict the performance of typical geometry features $(7,8,9,10,11,2)$. Such features would include sudden expansion chambers, extended tubes, resonators, and perforated materials. Limited resources in the past caused designers to make many assumptions when linking muffler transmission loss to how it will actually perform in operation. With the advancement of numerical methods, such as FEM and CFD, other key contributors to the overall muffler performance are starting to be investigated more rigorously.

The role of temperature effects are one of the driving influences on muffler performance. Both the speed of sound, which is the rate the sound pressure wave propagates through the medium, and the density of the medium are significantly 
influenced by temperature. The muffler can often be exposed to temperatures over $500{ }^{\circ} \mathrm{C}$ which causes the speed of sound to nearly double the speed at ambient conditions. Thus, the accuracy of the model is heavily dependent on the accuracy of the temperature profile. The TL of a muffler is traditionally calculated with baseline conditions which in itself can lead the designer down the wrong path. Keske modeled a simple expansion chamber using BEM techniques at an escaladed temperature by changing the speed of sound and density of the medium to match accordingly (6). The result was a shift in the TL which was proportional to frequency. Although the solution was trivial and no temperature gradient was applied to the model, the results showed a strong dependency on temperature and the accuracy of the temperature profile is crucial. The author has incorporated the effects of temperature gradients into FEM. The temperature profile was applied by breaking up the muffler in many discrete sections which was collected using experimental data. The findings showed that the temperature gradient not only shifted the frequency domain, but significantly altered the amplitude and shape of the TL.

Many more researchers have begun to use the time domain approach to solve computational fluid dynamics problems both in 1-D software such as GTPower and 3-D CFD software such as Fluent. Accurate temperature and velocity flow profiles as well as back pressure measurements have been achieved by both software packages with fairly good correlation to experimental methods. These temperature and flow velocity profiles then coupled to a frequency domain steady state solver, such as FEM, can accurately predict TL closer to operating condition.

Even better, if the source characteristics are known then the FEM model, including the flow characteristics, can be coupled to the source to predict effective sound pressure levels (SPL). Although the 1-D methods are much simpler to run, they tend to be only accurate in the low frequency range. The loss in accuracy is 
mainly due to the propagation of three dimensional waves which is not accounted for in the 1-D. The other noteworthy limitation to the 1-D method is that the muffler geometry often has to be simplified drastically. That said, the accuracy of the results of these investigations should be questioned heavily without correlation to experimental data.

Ji et al. incorporated mass flow rate at an elevated temperature in his muffler model built in GT-Power (12). As a temperature gradient was applied to the model, there was not only a frequency shift in the transmission loss but amplitude variations as well. The amplitude variations seemed to be unpredictable with respect to frequency although the overall amplitude level was fairly consistent. Ji et al. conducted a parametric study on the effects of varying the air flow rate through the muffler (12). The results showed a fairly substantial increase in TL (around 10 to $15 \mathrm{~dB}$ at a flow of $60 \mathrm{~m} / \mathrm{s}$ ) across the entire frequency band, predominantly at higher frequencies. The muffler of interest for this study was a three pass perforated tube muffler, which is one of the most common configurations in the automobile industry. These results defer from the theory which states that the convective flow effects of increased velocity has a tendency to decrease the attenuation characteristics of the muffler (2). The results achieved from this 1-D analysis can be questioned as there is no experimental data to correlate the results to and the results are not intuitive.

In the works of Siano et al., the TL of a three pass muffler configuration was calculated in both GT-Power using 1-D simulation and STS VNoise using 3D BEM (13). The acoustical BEM model was then coupled to a structural BEM model of the muffler to take into account the structural-fluid coupling. The perforated tubing was modeled in the BEM as an admittance property based on the semi-empirical formula developed by Sullivan and Crocker (14). The assigned admittance property allows the perforated holes to not have to be modeled which 
significantly reduces the element count for the model. In addition, modeling the perforated tubing as an admittance property allows the designer to easily change the perforate tube parameters to conduct a parametric study.

The 1-D TL results achieved by Siano et al. from GT-Power had fairly good correlation up to around $450 \mathrm{~Hz}$ (13). Above $450 \mathrm{~Hz}$, higher order modes began to propagate which were not accounted for in the model. When coupling the BEM to the structural model, the TL was fairly unchanged except for areas where resonances of the muffler were located. At resonance frequencies the attenuation of the muffler decreased significantly. It is noted that the areas where the resonances occur have a very small narrow band effect on the muffler TL (less than $5 \mathrm{~Hz}$ bandwidth). Also, the temperature of the muffler during operating conditions has a minor effect on structural resonances. Again, an accurate temperature profile on the structure is required for correct representation.

Siano et al. incorporated mass flow and temperature profiles to the GTPower model (13). The additional effects found that the increase in mass flow leveled out the peaks and troughs of the TL and the increase in temperature caused a significant frequency shift. Due to the limitation of BEM not being able to include mass flow, there was not an additional correlated model for this scenario. The use of FEM would allow for flow in the model although secondary effects such as flow noise would not be accounted for. In which case, it would be ideal to include a coupled CFD-FEM model for proper correlation of mass flow and temperature profiles.

Anderson implemented mass flow into the FEM indirectly by incorporating the effects into the boundary conditions (15). Specifically, the semi-empirical formula for the impedance of perforates developed by Sullivan and Crocker was modified to include the effects of mass flow (14). The flow resistivity term for the flow effects was added to the real portion of the impedance and the imaginary 
portion was unaltered (15). The implemented FEM code assumed constant temperature throughout the system. Since the mass flow effects were built into the boundary conditions (the impedance of the perforated tubing) no mass velocity was applied. Given the simulation setup, only experimental results with ambient conditions for correlation is practical. It is assumed that only considering mass flow effects on perforates alone will lead to inaccurate results. Anderson built a flow bench to correlate his simulated results that included a centrifugal fan and six loud speakers (15). The results achieved showed a high level of correlation for perforated tubing without flow applied. Poor correlation was achieved when the muffler was filled with absorptive material. This could have been due to an inaccurate impedance model that the author used. The incorporation of mass flow leveled out the peaks and troughs of the transmission loss. However, mass velocities only up to $30 \mathrm{~m} / \mathrm{s}$ were used due to the limitation of the equipment. This speed is approximately two times less than a typical mass velocity of an engine.

The works done by Sen presented a strategical method that uses multiple CFD techniques to predict sound pressure levels inside the muffler (16). Also included during the initial design phase was back pressure prediction defined as the difference in the static pressure in the exhaust pipe to the static pressure at the outlet of the tail pipe. Back pressure is a major concern as it reduces the power of the engine resulting in a lower fuel economy (17). The author used the steady state Reynolds Averaged Navier-Stokes (RANS) method to determine the flow pattern inside the muffler along with the backpressure. The second step was to use the RANS solution achieved as an input into the Large Eddy Simulation (LES) turbulent model that determines turbulent flow fluctuations and acoustic pressure distribution. The Ffowcs Williams Hawkins (FW-H) equations are then solved to get sound pressure levels in the near field inside the muffler. The results of the back pressure and sound pressure level prediction were significantly different from the experimental data although the trend patterns were consistent. 
The recent work in muffler performance focuses on incorporating source information into the model. The most common and accurate method for incorporating source information usually contains some form of hybrid approach, including both CFD and FEM or BEM techniques. Yadav et al. took the simplified approach to determine source characteristics by using the empirical formula derived by Collow and Peat for calculation of source impedance $(18,19)$. The empirical formula for source impedance is shown in Equation 1-2. The source impedance is assumed to only be a function of the characteristic impedance of the exhaust pipe denoted as Y.

$$
Z_{s}=Y(0.707-j \cdot 0.707) \quad \text { Equation } 1-2
$$

After determining the source data, Yadav et al. created the mesh model of the muffler using BEM techniques and correlated the BEM TL to the experimental TL with a high level of correlation (18). After proving the validity of the BEM model, the next step the author took was to apply the source properties to the inlet. In the BEM model the source is applied as an equivalent impedance and volume velocity. The author determined the volume velocity by first applying a uniform velocity on the BEM inlet tube then measuring the pressure at a reference location. From that measurement the author determined a transfer function for the volume velocity and pressure. The second measurement was an experimental measurement of sound pressure at the reference location. The experimental reading was used to back calculate the volume velocity based on the transfer function previously calculated. This method has limitations as it assumes a linear system and the sound source is applied evenly across the nodal interface at the inlet as if acting as a rigid piston. Also, based on the one-port model of the exhaust system, the transfer function relating input velocity to sound pressure is a function of both the source and muffler impedance (20). As a result, the experimental data would have to be 
acquired with the prototype muffler which would defeat the purpose of the simulation model. From the researcher's investigation, the results only reveal decent correlation for the low frequencies. The author assumed the source information was not a function of the load or speed of the engine. Also, it was unclear whether the prototype muffler was attached for the experimental measurement. Without higher correlation or a further justification, the results should be questioned.

Zheng et al. used a coupled hybrid approach to incorporate source properties from the 1-D method to the muffler's 4-pole parameters obtained by the 3-D FEM techniques (21). The 4-pole parameters are the ratio of the state variables, pressure and mass particle velocity, at the inlet and outlet of the muffler that can be used to determine the TL of a muffler (6). The source properties, including the source impedance and sound pressure, were calculated in GT-Power by using the multi-load method based on the electro-acoustic analogy. The electroacoustic analogy makes the comparison of an exhaust system to a simplified electrical circuit. The comparison is that the source pressure is the voltage, the mass velocity is the current, and the acoustical impedance is the electrical impedance (2). By applying two loads to the engine model (by changing the length of the pipe), the source pressure and source impedance are calculated (21). Increasing the number of loads applied to the system results in an over-determined set of equations, thus increasing the accuracy of the calculated source information. Once the source properties were known, the 4-pole parameters were calculated from the FEM model in the same way TL is calculated. The model for this case did not include temperature and mass flow affects; the limiting factor for the accuracy of this model. The 4-pole parameters, much like TL, are influenced by these two parameters. 
The coupled model performed by Zheng et al. was done in GT-Power which was calculated using the fundamental finite volume time domain techniques that involves the continuity, momentum and energy equations (21). The source was modeled as an equivalent impedance and pressure and the muffler was modeled by the 4-pole parameters. The insertion loss was calculated after replacing the 4-pole parameters with a straight pipe and then taking the difference between the two sound pressure levels. The results showed an overall level for insertion loss verses engine speed, no frequency spectrums were given. This was most likely due to the inaccuracies of the 4-pole parameters not being calculated in actual operating conditions, resulting in poor correlation. It is also noted that the 4pole parameters would have to be calculated separately for each engine speed for the most accurate results. This would significantly increase simulation time. The author of this thesis has determined a muffler's 4-pole parameters for various operating speeds which became a very time consuming process.

The noise emitted from a muffler consists of three main components: the noise from the pulsating pressure waves due to the timing of the combustion process, the flow noise generated from the turbulent flow through the exhaust system, and the shell noise which is the result of sound pressure waves exciting the structure. For the most part, all previous research only considered the pulsating pressure waves. It has been shown through the works of Wiemeler et al. that flow noise can be a substantial contributor to decreased insertion loss (21). The author developed an empirical formula to predict flow noise levels in muffler components. The author also found that noise generation for monopole sources were linear, dipole sources were exponential, and quadrupole sources were exponential to the fourth power all with respect to Mach speed in the muffler (21). The researcher found through experimental results, that the flow noise generated behaves mainly as dipole sources for Mach values up to 0.25 but quickly becomes quadrupole sources as Mach becomes larger than 0.25 (21). 
The difficulty with predicting flow noise in simulation is that CFD analysis is only useful when considering quadrupole noise sources. The results achieved by Wiemeler et al. were dimensionless and mainly used to find locations where high mass velocities existed (21). The CFD analysis, performed by Wiemeler et al., found a bend in the exhaust pipe caused a local velocity of $0.30 \mathrm{Ma}$ (21). After adjusting the bend to produce a local velocity of $0.23 \mathrm{Ma}$, the experimental results indicated a $6 \mathrm{~dB}$ decrease in the sound power level emitted by the exhaust system. The works presented proved to be an effective tool in flow noise optimization as long as the researcher understands the limitations of the CFD simulation. 


\section{Chapter 2. Theory of Muffler Acoustics}

The underlying theory of muffler acoustics presented in this chapter is derived from the works of M.L. Munjal's classical book “Acoustics of Ducts and Mufflers" (2). The works of this thesis is a further extension to the work presented by Keske (6), where Munjal's theory was explained in detail. As a result, the theory will be briefly covered for material pertaining to this thesis. For further information on the theory refer to the previous two references.

This thesis uses the conventional unit system. The rectangular coordinate system is represented by $x, y$, and $z$ and the cylindrical coordinates are $r, \theta$, and $z$. The variable, $t$, represents the time vector. In the frequency domain, $f$ is the frequency in $\mathrm{Hz}$ and $\omega$ is the frequency in $r / s$.

\subsection{Theory of acoustic wave propagation}

A sound pressure wave is created due to an abrupt change in position of particles in a medium. The sound pressure wave that progresses through space is the result of a miniscule fluctuation of the ambient static pressure with regard to the overall magnitude of the ambient static pressure itself (23). In the frequency range of interest for muffler analysis, these sound pressure waves typically travel through the pipes of an exhaust system as plane waves. Inside the muffler itself three-dimensional wave propagation can exist, particularly at the mid to high frequency range. 


\subsubsection{Plane wave theory}

A plane wave is a wave that progresses along a single axis, for an exhaust pipe the direction would be along the length of the pipe. At the wave front of a plane wave the two state variables, sound pressure, $p$, and particle velocity, $u$, would be constant. This would be an ideal case where the assumption is the plane wave is progressing through an inviscid stationary medium and sound pressure is only a function of the longitudinal displacement and time. Using the generalized mass, momentum, and energy equations, the 1-D wave formula is derived as shown in Equation 2-1. The variables, $p$ is the acoustic pressure and $c$ is the speed of sound through the medium (2). The wave equation was factored to show the two different wave forms, a forward moving or "progressive" wave and a backward moving or "reflective" wave.

$$
\frac{\partial^{2} p}{\partial t^{2}}-c^{2} \frac{\partial^{2} p}{\partial x^{2}}=\left(\frac{\partial p}{\partial t}+c \frac{\partial p}{\partial x}\right)\left(\frac{\partial p}{\partial t}-c \frac{\partial p}{\partial x}\right)=0 \quad \text { Equation } 2-1
$$

Upon solving the partial differential equation in the factored form shown in Equation 2-1 and assuming the time dependence will take an exponential form, the 1-D solution for plane wave sound pressure propagation is shown in Equation 2-2. In the equation, $C_{1}$ is the coefficient of the progressive wave, $C_{2}$ is the coefficient of the reflective wave, $\omega$ is the frequency in radians, and $k$ is the wave number which is shown Equation 2-3 (2). The wave number is simply the frequency spectrum normalized over the speed of sound. From the equation of the first state variable, sound pressure $p$, it is shown that the magnitude of the sound pressure is the addition of both the forward and backward traveling waves. In Equation 2-3, $\lambda$ represents the wavelength of the acoustic wave.

$$
p(x, t)=\left(C_{1} e^{-j k_{0} x}+C_{2} e^{+j k_{0} x}\right) e^{j \omega t} \quad \text { Equation } 2-2
$$

where: 


$$
k_{o}=\frac{\omega}{c}=2 \pi / \lambda
$$

The second state variable, particle velocity $u$, can be solved using the same wave equation presented in Equation 2-1 and replacing $p$ with $u$ and then solving the momentum equations relating the two state vectors. The particle velocity can be described using the same coefficients presented in Equation 2-2 and is shown in Equation 2-4. In the equation, $Z_{o}$ represents the characteristic impedance of the medium defined as the ratio of the two state variables, sound pressure and particle velocity, and is shown in Equation 2-5. In Equation 2-5, $\rho_{o}$ is the density of the medium.

$$
u(x, t)=\frac{1}{z_{o}}\left(C_{1} e^{-j k_{o} x}-C_{2} e^{+j k_{o} x}\right) e^{j \omega t} \quad \text { Equation } 2-4
$$

where:

$$
Z_{o}=\frac{p}{u}=\rho_{o} c
$$

Equation 2-5

In the case for a muffler analysis, it is much more common to use the mass velocity, $v$, instead of the particle velocity as the second state variable. A conversion from particle velocity to mass velocity is done. The formula for mass velocity can be seen in Equation 2-6 (2). In this case, $Y_{o}$ is the characteristic impedance of the tube, which is more appropriate then the characteristic impedance of the medium. The characteristic impedance of the tube is shown in Equation 2-7. In this equation, $S$ represents the cross sectional area of the tube.

$$
v(x, y)=\frac{1}{Y_{o}}\left(C_{1} e^{-j k_{o} x}-C_{2} e^{+j k_{o} x}\right) e^{j \omega t} \quad \text { Equation 2-6 }
$$

where:

$$
Y_{o}=\frac{p}{v}=c / S
$$

Equation 2-7 
In actual operating condition there will be a mean velocity flow, $\bar{V}$, through the exhaust system which will be described by its Mach number, $M$, as shown in Equation 2-8. In order to account for the convective effects of the mean flow while still assuming an inviscid medium, the state variables need to be written as a function of $M$. Through the works of Munjal, the derived state variables for pressure and mass velocity are shown in Equation 2-9 and Equation 2-10, including the mass flow effects (2).

$$
\begin{array}{cc}
M=\bar{V} / c & \text { Equation 2-8 } \\
p(x, t)=\left(C_{1} e^{-\frac{j k_{0} x}{1+M}}+C_{2} e^{+\frac{j k_{0} x}{1-M}}\right) e^{j \omega t} & \text { Equation 2-9 } \\
v(x, t)=\frac{1}{Y_{o}}\left(C_{1} e^{-\frac{j k_{0} x}{1+M}}-C_{2} e^{+\frac{j k_{0} x}{1-M}}\right) e^{j \omega t} & \text { Equation 2-10 }
\end{array}
$$

For further derivations, including implementation of viscosity and including compliant walls, refer to Munjal's book (2).

\subsubsection{Three-Dimensional Wave propagation}

The point where the 1-D plane wave theory breaks down is known as the cut-off frequency, $f_{c}$. At this frequency, the acoustic pressure waves begin to propagate in dimensions other than along the longitudinal axis. In this scenerio, higher order modes need to be accounted for within the state variables. The new governing equation is the 3-D wave equation shown in Equation 2-11.

$$
\frac{\partial^{2} p}{\partial t^{2}}-c^{2} \nabla^{2} p=0
$$

where: 


$$
\nabla^{2}=\frac{\partial^{2}}{\partial x^{2}}+\frac{\partial^{2}}{\partial y^{2}}+\frac{\partial^{2}}{\partial z^{2}} \text { or } \frac{\partial^{2}}{\partial r^{2}}+\frac{1}{r} \frac{\partial}{\partial r}+\frac{1}{r^{2}} \frac{\partial^{2}}{\partial \theta^{2}}+\frac{\partial^{2}}{\partial z^{2}}
$$

Munjal presents the entire derivation for the acoustic pressure state variable for a cylindrical duct, thus only the equation will be presented for the cylindrical ducts as shown in Equation 2-12 (2). For the entire derivation refer to Keske's Dissertation or Munjal's book $(6,2)$.

$$
p(r, \theta, x, t)=\sum_{m=0}^{\infty} \sum_{n=0}^{\infty} J_{m}\left(k_{r, m, n} r\right) e^{j m \theta}\left(C_{1, m, n} e^{-j k_{x, m, n} x}+C_{2, m, n} e^{+j k_{x, m, n} x}\right) e^{j \omega t} \quad \text { Equation 2-12 }
$$

For the acoustic pressure state variable shown in Equation 2-12, $k_{r, m, n}$ represents the radial wave number, $k_{r}$, for the $(\mathrm{m}, \mathrm{n})$ mode and is shown in Equation 2-13. After calculating $k_{r, m, n}$, the longitudinal transmission wave number, $k_{z, m, n}$, can be calculated as shown in Equation 2-14. It should be noted that for plane wave propagation, $k_{r, m, n}$ is equal to zero, $\mathrm{k}_{z, m, n}$ is equal to $k_{o}$ and Equation 2-12 becomes Equation 2-2.

$$
\begin{gathered}
k_{r}^{2}=k_{o}^{2}-k_{z}^{2} \\
k_{z, m, n}=\sqrt{k_{o}^{2}-k_{r, m, n}^{2}}
\end{gathered}
$$$$
\text { Equation 2-13 }
$$

The variable, $J_{m}$, in Equation 2-12 represents the Bessel function of the first kind of order $\mathrm{m}$. Imposing a zero radial velocity boundary condition at the cylindrical walls requires the first derivative of the Bessel function to be equal to zero. Given the boundary condition, the Bessel function, $\beta_{m, n}$, and the roots for the Bessel function derivative, $\alpha_{m, n}$, for cylindrical ducts were tabulated for each mode number up to mode $(5,5)$ in Keske's dissertation (6). It has been shown that 
higher modes will begin to propagate in a cylindrical duct at a frequency governed by Equation 2-15, where $\mathrm{D}$ is the diameter of the cylindrical duct.

$$
f=\frac{\alpha_{m, n} c}{\pi D} \quad \text { for } m, n=0 \text { to } \infty \quad \text { Equation } 2-15
$$

Keske's tabulated results showed the first two roots, $\alpha_{0,1}$ and $\alpha_{1,0}$ of the Bessel Function Derivative have values of 1.841 and 3.832, respectively (6). By using Equation 2-15 and assuming ambient conditions (where the speed of sound is calculated to be $340 \mathrm{~m} / \mathrm{s}$ ), the cut-on frequency can be determined. The cut-on frequency for the first diametrical and axisymmetric higher order mode effects of a cylindrical tube can be seen in Figure 2.1. If the inlet and outlet tube are symmetric about the center then the effects of the diametrical modes will not affect TL.

A demonstration of how plane waves and three dimensional waves propagate in an empty expansion cylindrical chamber is shown in Figure 2.2. A

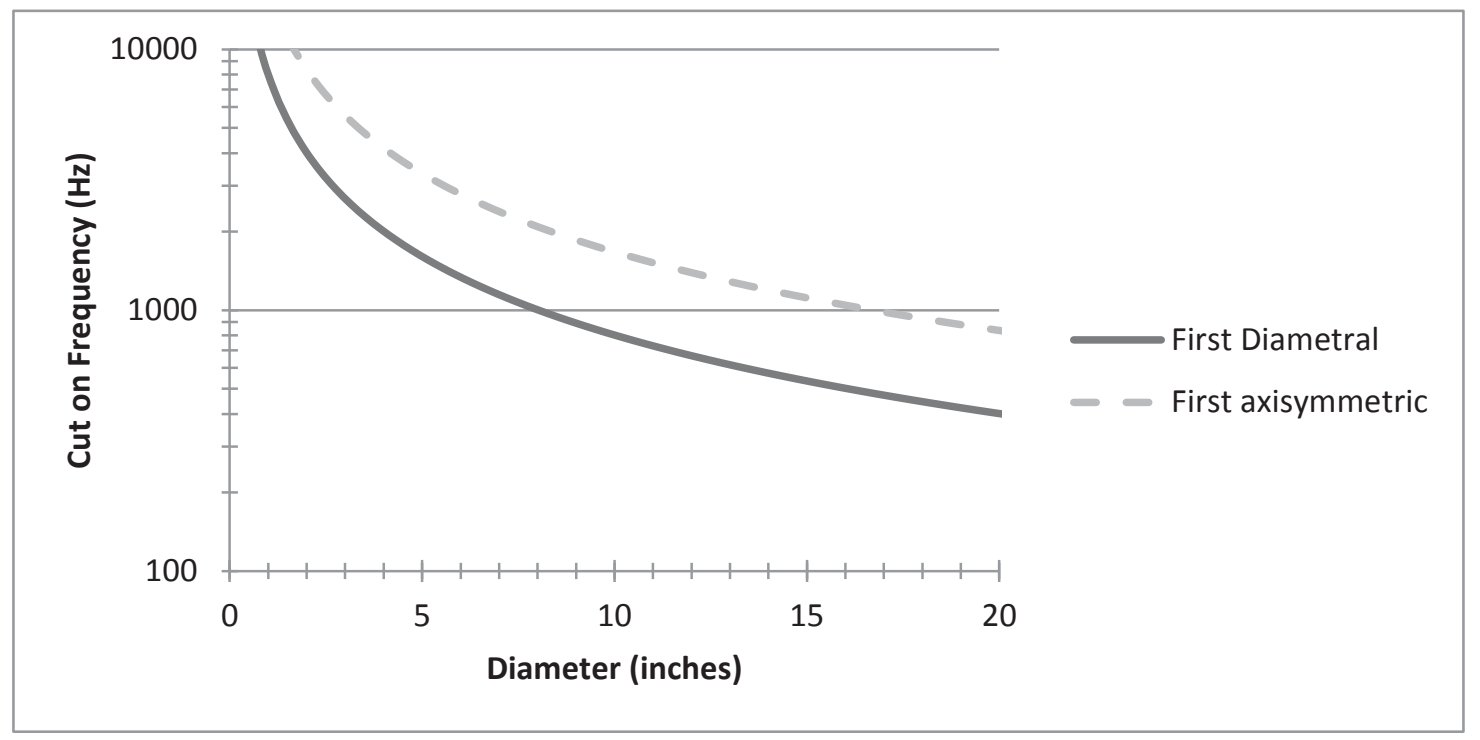

Figure 2.1: The cut-on frequency of higher order mode effects in a cylindrical muffler as a function of diameter. 
(a)

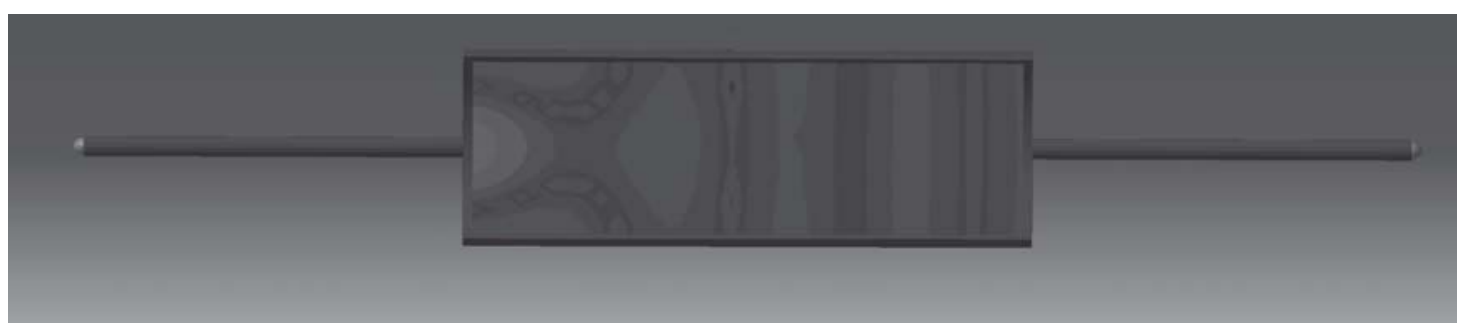

(b)

Figure 2.2: (a) Plane wave propagation at $400 \mathrm{~Hz}$ and (b) 3-D wave propagation at $1800 \mathrm{~Hz}$ in an empty eight inch diameter chamber.

muffler with a diameter of 8 inches and a length of 18 inches was used in the figure. In Figure 2.2, (a) represents a plane wave with a frequency of $400 \mathrm{~Hz}$ and (b) represents a three-dimensional wave at a frequency of $1800 \mathrm{~Hz}$.

\subsection{Transfer Matrix Method}

An acoustic filter can be defined as any element or combination of elements that are placed in the path of the source to the receiver. For the context of this thesis, the acoustic filter can be described as the exhaust system where mass flow and temperature gradients are not being considered. Aeroacoustic filters incorporate mass flow and temperature effects which are described in full within Munjal's book (2). The author believes it is necessary to initially neglect these effects to fully understand how these individual elements behave. Therefore, only 
the idealized case of no mass flow and temperature gradients will be presented in this section.

In order to fully describe how elements behave, it is necessary to define the relationship between the two state variables, $p$ and $v$, both upstream and downstream of the element. The most effective and practiced approach is through the use of the transfer matrix method. This method determines the "4-pole" parameters of the element. The 4-pole parameters are the variables $A, B, C$, and $D$. Using this method, each individual muffler element as their own 4-pole parameters. The major benefit of the transfer matrix is that individual elements can be combined through matrix multiplication to determine the global 4-pole parameters. The global transfer matrix can then be used to fully quantify the acoustic properties of the filter. A sample diagram indicating the locations of the state vectors for a two element filter is shown in Figure 2.3. The equation for calculating the local 4-pole parameters for the first element, $i 1$, is shown in Equation 2-16. Equation 2-17 shows the calculation for the global 4-pole parameters for this two element filter. For an acoustic filter with an element size $n$, there are $n$ local transfer matrices needed to determine the global 4-pole parameters.

$$
\begin{gathered}
{\left[\begin{array}{l}
p_{i} \\
v_{i}
\end{array}\right]=\left[\begin{array}{ll}
A_{i 1} & B_{i 1} \\
C_{i 1} & D_{i 1}
\end{array}\right]\left[\begin{array}{l}
p_{1} \\
v_{1}
\end{array}\right]} \\
{\left[\begin{array}{c}
p_{i} \\
v_{i}
\end{array}\right]=\left[\begin{array}{ll}
A_{i 1} & B_{i 1} \\
C_{i 1} & D_{i 1}
\end{array}\right]\left[\begin{array}{ll}
A_{1 o} & B_{1 o} \\
C_{1 o} & D_{1 o}
\end{array}\right]\left[\begin{array}{c}
p_{o} \\
v_{o}
\end{array}\right]=\left[\begin{array}{ll}
A & B \\
C & D
\end{array}\right]\left[\begin{array}{c}
p_{o} \\
v_{o}
\end{array}\right]}
\end{gathered}
$$




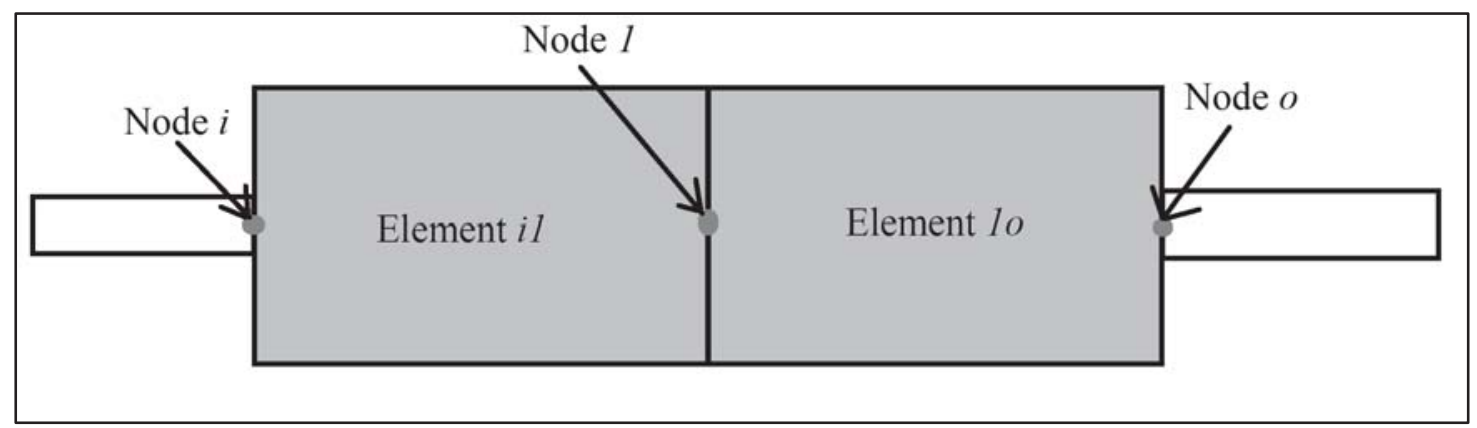

Figure 2.3: Location of the state vectors $p, v$ for the two element acoustic filter.

The other major benefit of using the transfer matrix method is that nearly all types of elements used in the application of an exhaust system can be categorized as one of three different types of elements: a distributed element, inline lumped element, and a shunt lumped element (6). A distribution element represents a uniform tube. In the case of an exhaust system, it would describe the exhaust pipe (upstream of the muffler) and the tailpipe (downstream of the muffler) as well as any section in the muffler that represents a uniform tube. An in-line lumped element signifies a sudden area change. This would be the case for an expansion into a chamber or the compression from the muffler to the tailpipe. A shunt lumped element represents a Helmholtz resonator, or more popular in the application of an exhaust system, a quarter-wave resonator. The transfer matrices for the three elements are shown in Equation 2-18 through Equation 2-20. In these equations, the subscript $e$ represents "element" and $l$ is the length of the element. A diagram showing the three basic types of elements is shown in Figure 2.4. It should be noted from Figure 2.4, that for the lumped in-line and lumped shunt elements, the locations to the left and right of the io points are simply distribution elements.

(Distributed Element) $\left[\begin{array}{cc}\cos \left(k_{o} l_{e}\right) & \left(j Y_{e}\right) \sin \left(k_{o} l_{e}\right) \\ \left(j / Y_{e}\right) \sin \left(k_{o} l_{e}\right) & \cos \left(k_{o} l_{e}\right)\end{array}\right] \quad$ Equation 2-18 
(Lumped in-line Element)

(Lumped shunt Element) $\left[\begin{array}{cc}1 & Z_{e} \\ 0 & 1\end{array}\right]$

$\left[\begin{array}{cc}1 & 0 \\ 1 / Z_{e} & 1\end{array}\right]$
Equation 2-19

Equation 2-20

The most used comparison that can be made when looking at the acoustical design of an exhaust system is the relationship of the acoustical transmission networking to an electrical circuit (2). The analogy comparison is shown in Table 2.1. A diagram of the acoustical transmission network including the source and termination properties is shown in Figure 2.5. The muffler itself is shown as a "black box" in the figure. The actual transmission network of the filter is not shown. Figure 2.6 shows how the networking of the distributed, lumped in-line, and lumped shunt elements are configured as well as the connections between the elements.

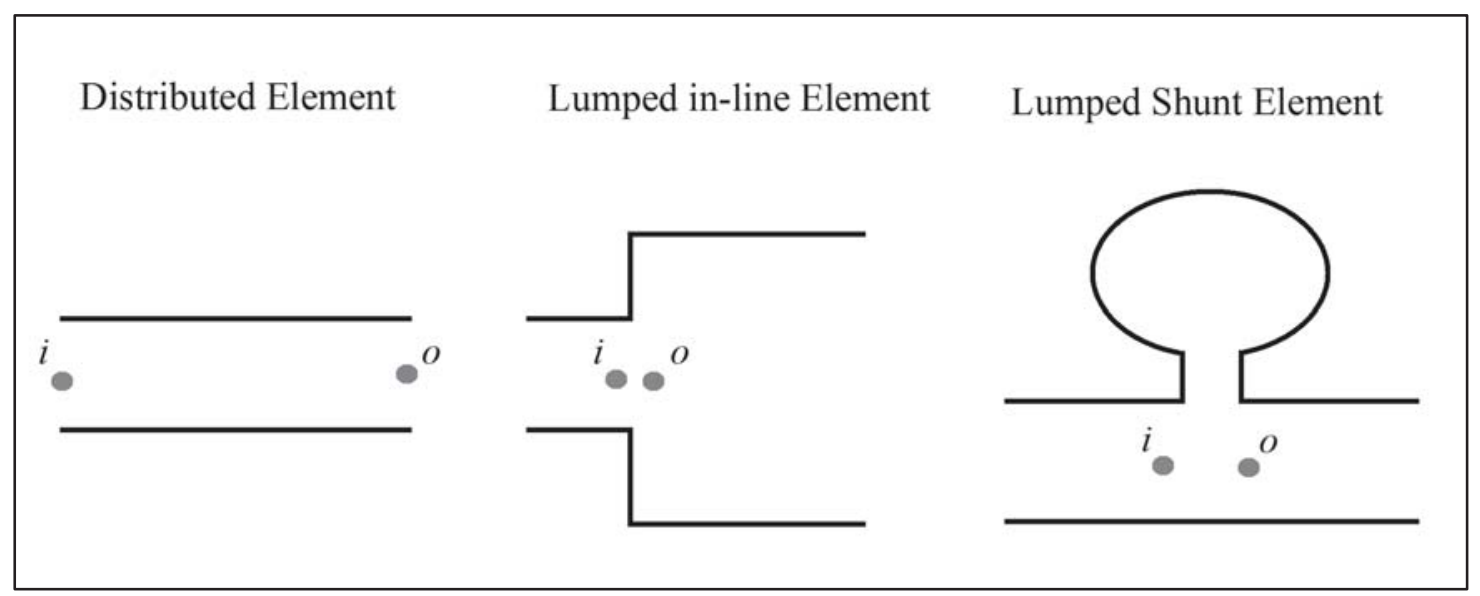

Figure 2.4: The three basic types of elements in an exhaust system using the transfer matrix method. 
Table 2.1

The comparison of an acoustical transmission network with the corresponding electrical circuit analogy.

\begin{tabular}{|l|c|l|c|}
\hline Acoustical Transmission Network & \multicolumn{2}{|c|}{ Electrical Circuit Network } \\
\hline Acoustical Pressure & $\boldsymbol{p}$ & Voltage & $\boldsymbol{V}$ \\
\hline Mass veleocity & $\boldsymbol{v}$ & Current & $\boldsymbol{i}$ \\
\hline Acoustical Impedance & $\boldsymbol{Z}$ & Electrical Impedance & $\boldsymbol{Z}_{\text {elec }}$ \\
\hline Resistance & $\boldsymbol{R}$ & Resistance & $\boldsymbol{R}_{\text {elec }}$ \\
\hline Inertance & $\boldsymbol{M}$ & Inductance & $\boldsymbol{L}$ \\
\hline Compliance & $\boldsymbol{C}$ & Capacitance & $\boldsymbol{C}_{\text {elec }}$ \\
\hline
\end{tabular}

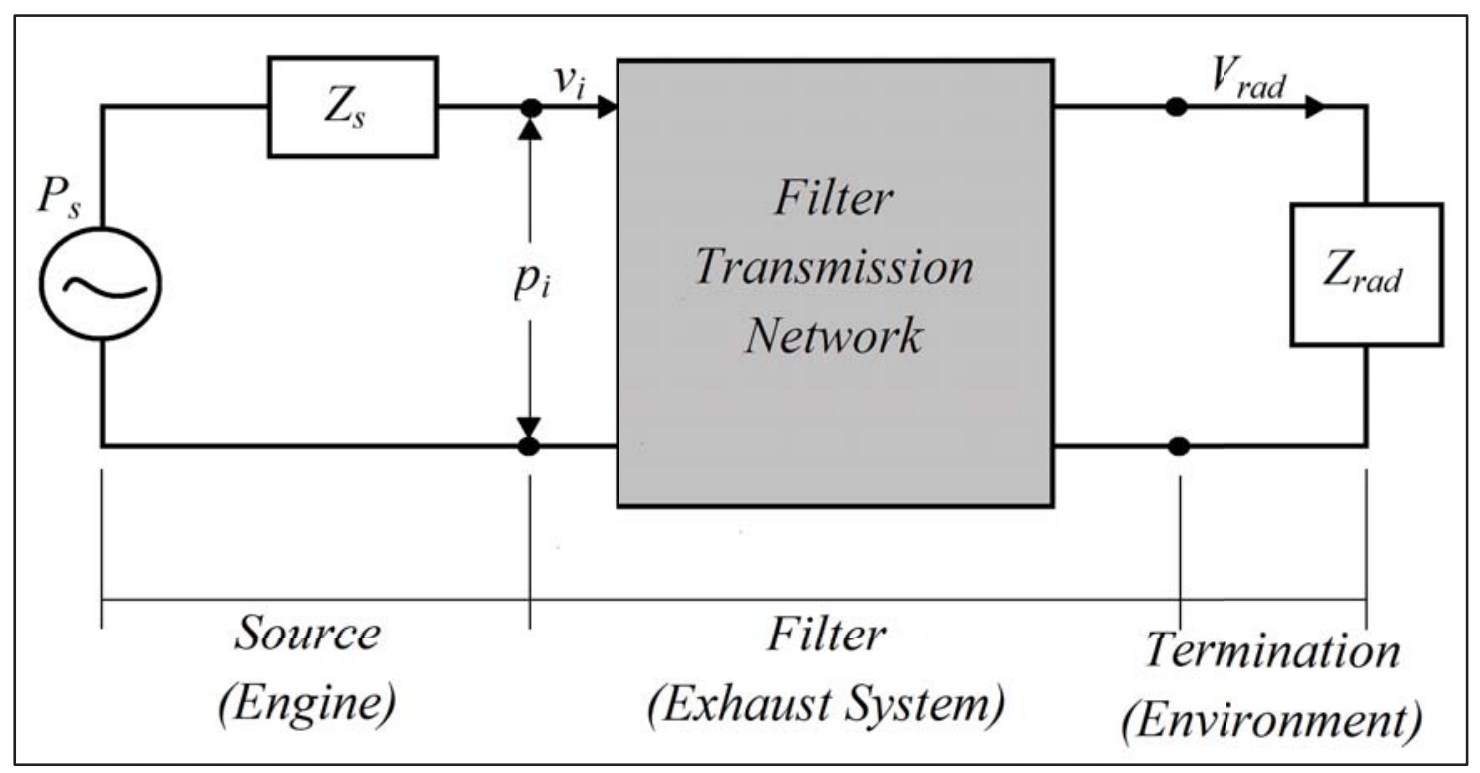

Figure 2.5: Acoustical Transmission network of an entire exhaust system including acoustical source and termination where networking for the filter is not depicted. 


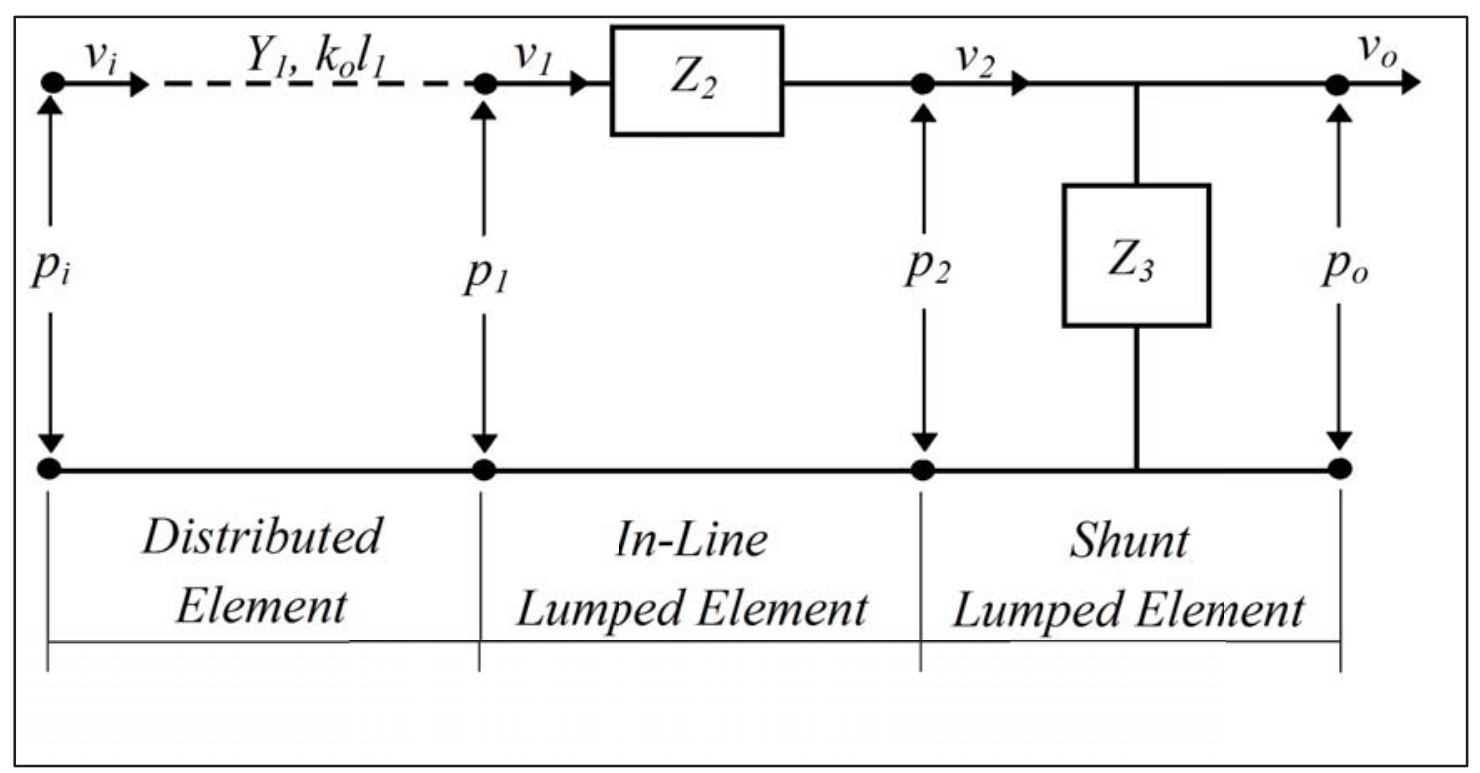

Figure 2.6: Acoustical Transmission network for the three different elements used in acoustic filters.

\subsection{Muffler Performance Parameters}

The performance of the exhaust system can be characterized in a variety of ways. However the proper selection of the performance parameter is essential to properly draw conclusions on the overall effectiveness of the exhaust system. The most commonly used performance parameter is Transmission Loss (TL). As this parameter is a function of the muffler alone, the source and termination properties do not need to be defined. The second parameter is Insertion Loss (IL), which is the difference in acoustic powers radiated from the exhaust system with the muffler attached and with the muffler replaced by a straight pipe. The IL is measured in actual operation. Because of this, the source and termination properties must be known. Experimentally this is a very straight forward process although predicting this process numerically can be very exhaustive. The final parameter is the Level Difference (LD) or commonly referred to as "Noise 
Reduction (RD)". This is the overall decrease in sound pressure levels with and without the muffler attached at any two arbitrary points in the exhaust system (3).

\subsubsection{Transmission Loss}

Transmission Loss, $T L$, is the difference in the acoustical power of the forward traveling "incident" pressure wave at the inlet of the muffler to the forward traveling "transmitted" pressure wave at the outlet (3). Transmission Loss requires an anechoic termination at the outlet which results in having no reflective waves in the outlet tube. This is not the case in the inlet tube. It will be shown in a later chapter that for experimental analysis having an anechoic termination is unfeasible, thus testing needs to be done in two configurations. The equation for $T L$ is shown in Equation 2-21 where the subscripts 1 and 2 represent the total measured sound pressure (including reflective waves) at the inlet and outlet, respectively (21).

$$
T L=20 \log \left(\left|\frac{p_{\text {inc. }}}{p_{\text {trans. }}}\right|\right)+10 \log \left(\frac{S_{o}}{S_{i}}\right)=20 \log \left(\left|\frac{p_{1}+\rho c v_{1}}{2 p_{2}}\right|\right)+10 \log \left(\frac{S_{o}}{S_{i}}\right) \text { Equation 2-21 }
$$

In Equation 2-21, $\rho c$ represents the characteristic impedance of the medium, $v_{1}$ is the applied particle velocity at the inlet, and $S_{i}, S_{o}$ represent the cross sectional areas of the inlet and outlet, respectively. Usually the cross-sectional area of the inlet and outlet are identical. As a result, the second term in the TL can be removed. Using FEM techniques, this formulation of TL can be very useful. The particle velocity at the inlet can be defined as a known boundary condition (usually a unit vector) and an anechoic termination can be easily modeled. Using the FEM, only a total of two measurement points are required for the calculation of TL. This is not true experimentally as the mass velocity in the inlet tube cannot 
be determined using a single measurement point. It is also not feasible to recreate an anechoic termination. Thus, experimentally it is ideal to incorporate the 4-pole parameters into the TL calculation. The derivation of TL using the 4-pole parameters was done by Munjal (2). Making the necessary assumptions that the TL was measured with no temperature gradients, the TL with the 4-pole parameters can be simplified to the equation shown in Equation 2-22 (2).

$$
T L=20 \log _{10}\left(\left|\frac{1}{2}\left[A+\frac{B}{\rho_{o} c}+\left(\rho_{o} c\right) C+D\right]\right|\right)+10 \log _{10}\left(\frac{S_{o}}{S_{i}}\right) \quad \text { Equation 2-22 }
$$

\subsubsection{Insertion Loss}

As stated previously, Insertion Loss, $I L$, is the difference in acoustical power of the exhaust system with the muffler attached and then replaced by a straight pipe (3). The equation of $I L$ can be seen in Equation 2-23. In the equation, $L_{w}$ is the sound power level in decibels $(\mathrm{dB})$ whereas $\mathrm{W}$ is the sound power level in Pascal's (Pa). The subscript 1 represents the straight pipe configuration and subscript 2 represents the muffler configuration.

$$
I L=L_{w 1}-L_{w 2}=10 \log _{10}\left(\frac{W_{1}}{W_{2}}\right)
$$

Equation 2-23

The derivation of IL was done by Munjal and can be seen in Equation 2-24 (2). From the equation, the subscript $o$ represents the location just outside of the tailpipe in the atmosphere and the subscript $s$ represents the source. In the formula, $R$ represents the real or "resistive" part of the characteristic impedance, $Z$. Assuming that the density of the atmosphere just outside of the tailpipe with and without the muffler installed is relatively constant, the first term in the logarithm is 
equal to one. Also, it has been shown in the works of Munjal that the real part of the radiation impedance is primarily a function of the tailpipe radius (2). This allows the second part of the logarithm to equal one. With these assumptions, IL can be reduced to Equation 2-25. The last term, $V R$, represents the velocity ratio between the source and radiation. As shown in Equation 2-25, with the given assumptions IL becomes a function of the source impedance, the radiation impedance of the straight pipe configuration, and the velocity ratio between the source and the radiation exiting the tailpipe. The velocity ratio is a function of the four-pole parameters of the muffler and the radiation impedance of the muffler configuration to the environment (6). Due to the required knowledge of source and termination properties, this type of calculation can be very difficult to predict numerically. As a result, IL is much more commonly determined experimentally.

$$
\begin{gathered}
I L=20 \log _{10}\left(\left(\frac{\rho_{r a d, 2}}{\rho_{\text {rad }, 1}}\right)^{\frac{1}{2}}\left(\frac{R_{r a d, 1}}{R_{r a d, 2}}\right)^{\frac{1}{2}}\left|\frac{Z_{S}}{z_{s}+Z_{r a d, 1}}\right|\left|\frac{v_{s}}{v_{r a d}}\right|\right) \\
I L=20 \log _{10}\left(\left|\frac{Z_{S}}{z_{S}+Z_{r a d, 1}}\right|\left|V R_{s, r a d}\right|\right)
\end{gathered}
$$

Equation 2-25

\subsubsection{Level Difference}

Level Difference is the overall noise reduction of an exhaust system at any arbitrary point in the inlet of the muffler to an arbitrary point in the outlet of the muffler and can be seen in Equation 2-26 (3). Because of its simplicity and as it does not distinguish between transmitted and reflective waves, its usefulness is fairly limited except for comparison purposes. In a later section, the author used this method to compare the flow noise generated inside of the exhaust system for a variety of muffler configurations which proved to be a useful analysis. 


$$
L D=20 \log _{10}\left(\frac{p_{i}}{p_{o}}\right)
$$

Equation 2-26

\subsection{Theory of acoustic filters applied to mufflers}

When examining many different muffler configurations in a variety of industries, it becomes apparent that they all use the same types of elements. The elements which are commonly used are uniform tubes, extended tubes, Helmholtz resonators, perforated tubing, and absorption materials. This section will describe the function of each of these elements.

\subsubsection{Uniform tube}

A uniform tube can be described as a distribution element as long as plane wave propagation exists. For a circular tube of diameter, $D$, plane wave propagation exists provided that the criteria in Equation 2-27 is satisfied. It should be noted that for the TL equations to be valid, plane waves must be present in the exhaust and tail pipe. If this is the case, the 4-pole parameters of the element can be calculated using Equation 2-18 which can then be used to calculate TL.

$$
f>\frac{1.841}{\pi D} c_{o}
$$

Equation 2-27 


\subsubsection{Extended tube resonators}

Extended tube resonators are very common in muffler design because of their ability to cause acoustical resonances in the muffler at specific frequencies, which is a function of the length of the extended tube. In a transmission network diagram, extended tube resonators can be described as a lumped shunt element as shown in Figure 2.4. When the impedance tends to zero no acoustical energy is transmitted and there is a large peak in TL (2). In which case, this is the frequency where the acoustical resonance occurs. The results from this thesis show that the resonances improve the TL in a fairly wide frequency span centered around the resonance. This can be very beneficial to see the effects of the resonance at many operating conditions.

In order to describe where in the frequency spectrum the resonance is located, it is necessary to describe what type of extended tube resonator is being used. There are two different types of extended tube resonators as can be seen in Figure 2.7. The parameter of interest is $L$, which causes a structural resonance at frequencies described in Equation 2-28 and is where a quarter wavelength of the sound wave is equal to $L$. This type of element is commonly referred to as a quarter wave resonator.

$$
f=\frac{c}{4 L}(2 n+1) \quad \text { for } \quad n=0,1,2, \ldots \quad \text { Equation } 2-28
$$




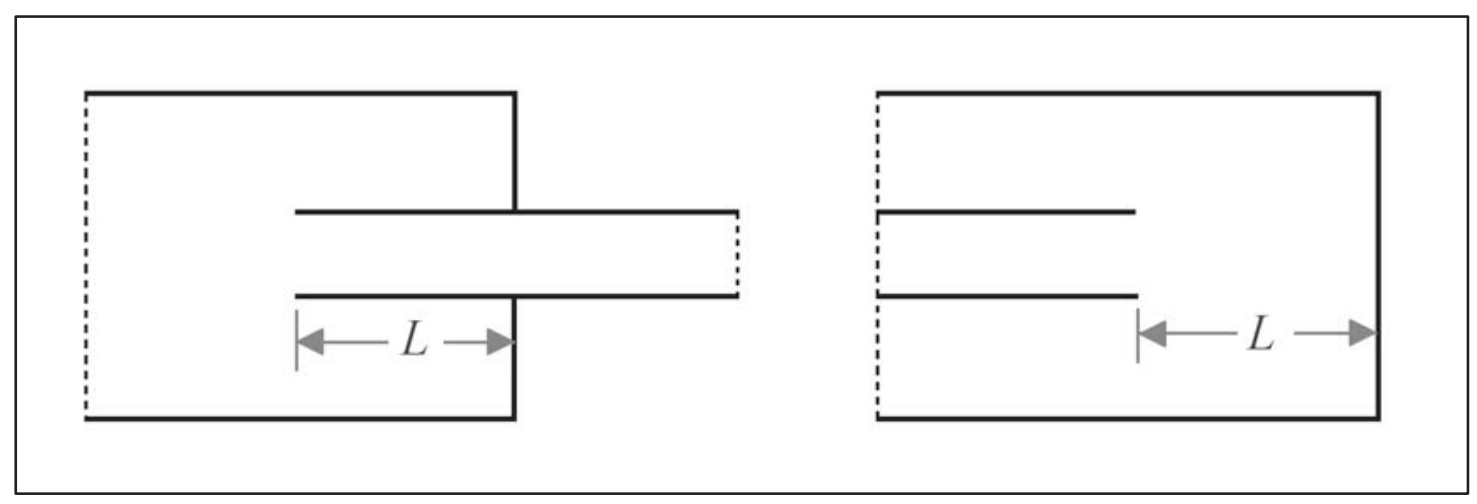

(a)

(b)

Figure 2.7: Quarter wave resonator diagrams for (a) extended inlet/outlet and (b) flow reversal (expansion/contraction)

\subsubsection{Helmholtz and Concentric hole-cavity resonator}

A Helmholtz resonator is modeled as a lumped shunt element and can be seen in Figure 2.8. Much like the quarter wave resonators shown in the last section, these elements are tuned to a certain frequency which causes an acoustical resonance. The equivalent impedance of the resonator can be calculated using Equation 2-29. In this equation, $t_{w}$ is the tube thickness, $L_{e q}$ is the equivalent length of the neck, and $S_{n}$ is the neck cross sectional area.

$$
Z=\left(\frac{\omega^{2}}{\pi \cdot c}\right)+j\left(\omega \frac{l_{e q}}{S_{n}}-\frac{c^{2}}{\omega V_{o}}\right)
$$

where:

$$
\begin{gathered}
l_{e q}=L_{n}+t_{w}+.85 \cdot D_{n} \\
S_{n}=\frac{\pi}{4} D_{n}^{2}
\end{gathered}
$$




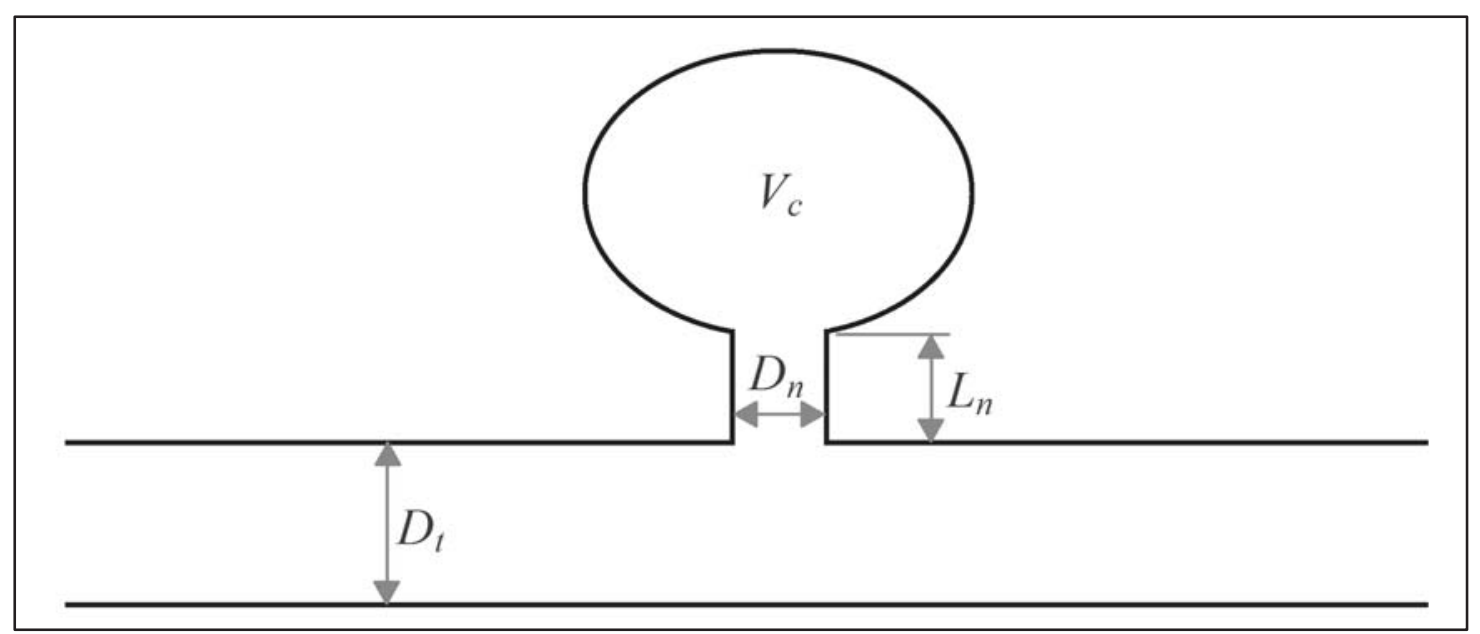

Figure 2.8: Diagram of a Helmholtz resonator.

From Equation 2-29, the 4-pole parameters of the element can be determined which can be used to determine the Transmission Loss of the element using Equation 2-22. The calculation for TL can be seen in Equation 2-30 where $Z_{r l}$ is the real portion of the impedance and $Z_{i m g}$ is the imaginary portion. An example of the TL of a Helmholtz resonator is shown in Figure 2.9, where the parameters are given in Table 2.2.

$$
T L(d B)=20 \log _{10}\left(\sqrt{\left(2+\left(\frac{c}{S_{o}}\right) \frac{Z_{r l}}{z_{r l}^{2}+Z_{i m g}^{2}}\right)^{2}+\left(2+\left(\frac{c}{S_{o}}\right) \frac{Z_{r l}}{z_{r l}^{2}+Z_{i m g}^{2}}\right)^{2}} / 2\right)
$$

Table 2.2

Sample Helmholtz resonator properties used for calculation of Transmission Loss.

\begin{tabular}{|c|c|l|}
\hline \multicolumn{3}{|c|}{ Helmholtz resonator properties } \\
\hline Volume of Cavity, $\boldsymbol{V}_{\boldsymbol{c}}$ & 10 & in2 \\
\hline Neck Length, $\boldsymbol{L}_{\boldsymbol{n}}$ & 1 & in \\
\hline Neck Diameter, $\boldsymbol{D}_{\boldsymbol{n}}$ & 1 & in \\
\hline Tube Diameter, $\boldsymbol{D}_{\boldsymbol{t}}$ & 1 & in \\
\hline Tube Thickness, $\boldsymbol{t}_{\boldsymbol{w}}$ & 0.1 & in \\
\hline Speed of Sound, $\boldsymbol{c}$ & 348 & $\mathrm{~m} / \mathrm{s}$ \\
\hline
\end{tabular}




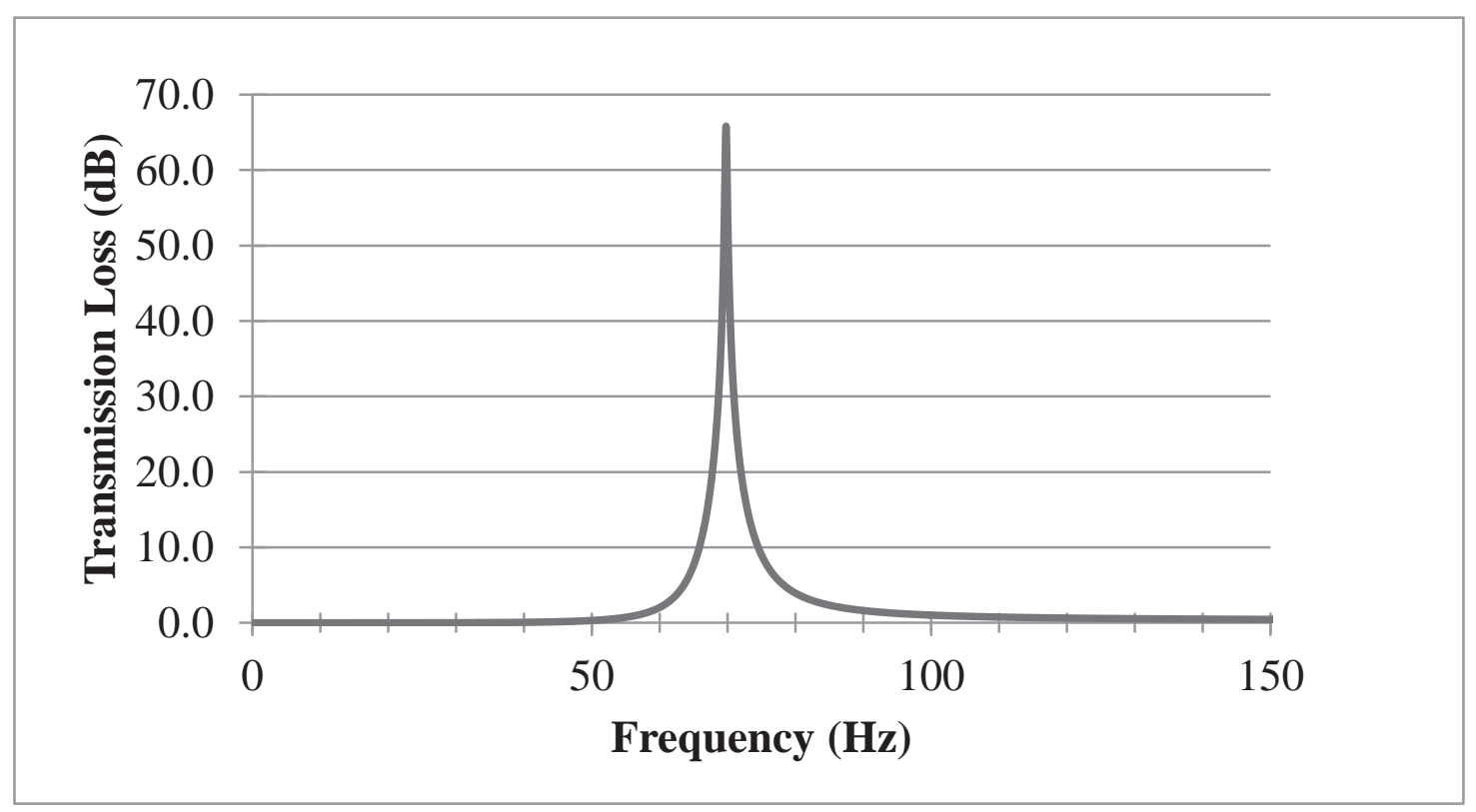

Figure 2.9: Transmission Loss of a simple Helmholtz resonator.

A more popular form of a Helmholtz resonator in muffler design is a concentric tube resonator. The concentric tube resonator is a tube that contains a series of perforations in a specific location that opens into an expansion tube. A diagram showing the concentric tube resonator is shown in Figure 2.10. This type of resonator is more ideal for muffler design for several reasons. The performance of the resonator is significantly more efficient when there is flow in the exhaust system. The space requirements are also less due to its concentric shape and the transmission loss has a slightly higher broadband performance due to the resistivity of the perforated tubing. The equation used to calculate the equivalent impedance is shown in Equation 2-31. In the equation, $n_{h}$ represents the number of holes in a circumferential row, $d_{h}$ is the diameter of the perforated holes, $t_{w}$ is the wall thickness, $l_{e q}$ is the equivalent length, $S_{h}$ is the surface area of each hole, $S_{c}$ is the cross sectional area of the expansion tube, and $Y_{c}$ is the characteristic impedance of the expansion tube. The same calculation shown in Equation 2-30 can be used to calculate TL. 


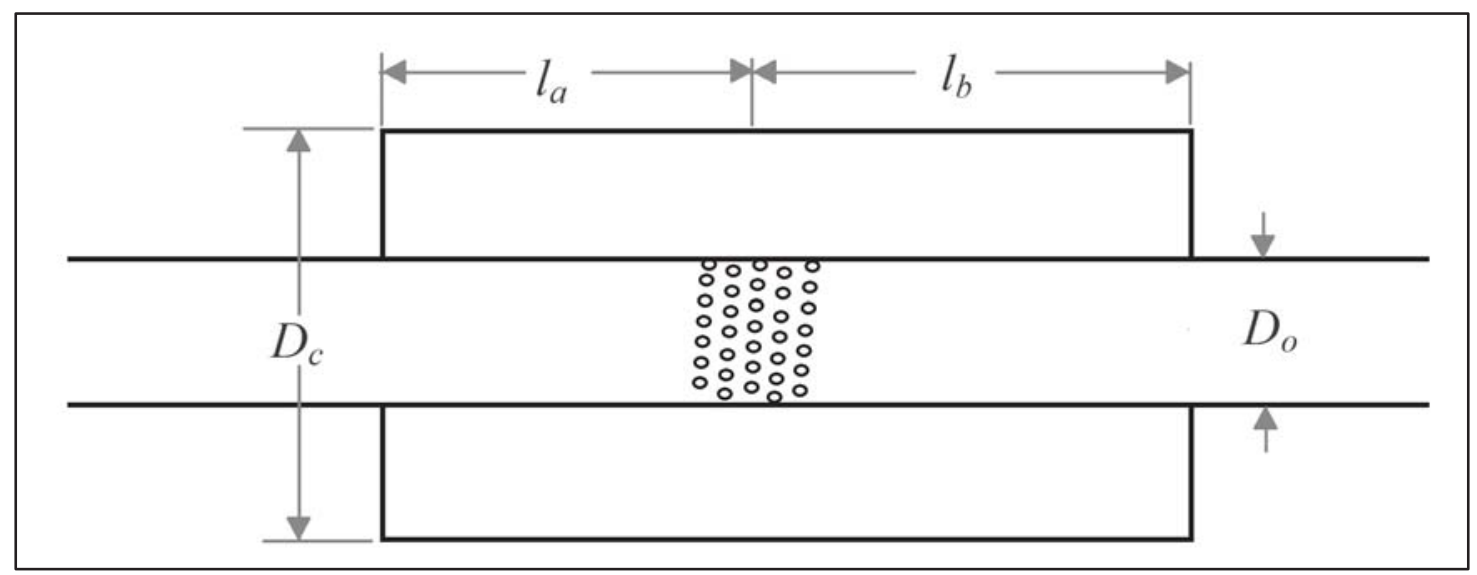

Figure 2.10: Diagram of a concentric tube resonator.

$$
Z=\left(\frac{\omega^{2}}{n_{h} \cdot \pi \cdot c}\right)+j\left(\left(\frac{\omega \cdot l_{e q}}{n_{h} \cdot S_{h}}\right)-\frac{Y_{c}}{\tan \left(k_{o} \cdot l_{a}\right)+\tan \left(k_{o} \cdot l_{b}\right)}\right) \quad \text { Equation 2-31 }
$$

where:

$$
\begin{gathered}
l_{e q}=t_{w}+0.85 \cdot d_{h} \\
S_{h}=\frac{\pi}{4} d_{h}^{2} \\
S_{c}=\frac{\pi}{4}\left(D_{c}^{2}-D_{o}^{2}\right) \\
Y_{c}=\frac{c}{S_{c}}
\end{gathered}
$$

\subsubsection{Perforated tubing}

Perforated tubing is in nearly all commercial mufflers and is quite beneficial when large flow velocities are seen inside of the muffler. When an exhaust stream exits out of a tube within the muffler there is typically a flow jet that forms (6). In order to combat this phenomenon, perforated tubing is used to steady the flow and force the flow to expand into the entire chamber. Perforated tubing can also be considered a dissipative element. When the acoustical pressure 
wave grazes past the perforations it feels a resistance which converts the acoustical energy into heat (12). The driving parameter in the performance of the perforated section is the equivalent impedance, $Z_{\text {perf }}$. The perforated impedance consists of a real part, $R_{\text {perf }}$, (resistance) and an imaginary part, $X_{\text {perf }}$, (reactance). This is shown in Equation 2-32.

$$
Z_{\text {perf }}=R_{\text {perf }}+j \cdot X_{\text {perf }}
$$

Equation 2-32

The calculation for the resistance and reactance of the perforated impedance is seen in Equation 2-33 and Equation 2-34, respectively (24). These can be used for both sheets and tube. These equations hold true in the linear range and when the sheet is not too thick. In the equations $\varepsilon$ represents the porosity of the surface, $\eta$ is the dynamic viscosity of air which is primarily a function of temperature, $\rho_{o}$ is the density of the medium, $t$ is the surface thickness, $R_{h}$ is the radius of the holes, and $C F$ is a correction factor which is dependent on the hole pattern, spacing, and diameter. The author created a MatLab script that will calculate the perforation impedance and is shown in Appendix B.

$$
\begin{gathered}
R_{\text {perf }}=\frac{1}{\varepsilon} \sqrt{8 \cdot \omega \cdot \eta \cdot \rho_{o}}\left(1+\frac{t}{2 \cdot R_{h}}\right) \\
X_{\text {perf }}=\frac{1}{\varepsilon} \omega \cdot \rho_{o}(t+2 \cdot C F)
\end{gathered}
$$

Equation 2-33

Equation 2-34

Modeling small holes can be very exhaustive and requires a significant number of elements. This leads to an exponential increase in simulation time. Also, because the spacing of the perforated holes is very close together, the results achieved tend to be fairly inaccurate. It is often more convenient to model the perforated tubing as a continuous sheet and apply a transfer admittance property that relates the state variables $p$ and $v$ for the inner and outer face. This admittance 
relationship is shown in Equation 2-35; where $k$ is a correction factor that takes into account the thickness of the pipe (24). In the case of a perforated baffle or sheet, $k$ would equal to zero.

$$
\left[\begin{array}{c}
v_{\text {in }} \\
v_{\text {out }}
\end{array}\right]=\left[\begin{array}{cc}
1 / Z_{\text {perf }} & -1 / Z_{\text {perf }} \\
-k / Z_{\text {perf }} & k / Z_{\text {perf }}
\end{array}\right]\left[\begin{array}{c}
p_{\text {in }} \\
p_{\text {out }}
\end{array}\right]
$$

Equation 2-35

Many parametric studies have been performed on the effects of perforation properties $(3,12,14,22)$. All the results have revealed that by decreasing the porosity the TL is increased at all frequencies slightly. On the downside, as the percent open area is decreased below the cross sectional area of the tube, back pressure is affected considerably. The hole diameter had little to no effect on the analytical and experimental results achieved.

\subsubsection{Absorptive material}

The use of sound absorption material in an exhaust system serves to dissipate the energy of the acoustic waves into heat and also to store heat energy from the exhaust stream. Using absorptive material can greatly increase the transmission loss of an exhaust system in the mid to high frequency ranges. In the muffler system, however, the absorption material is typically not thick enough to perform attenuation of the low frequencies. For the material to provide attenuation, the material thickness needs to be at least $1 / 10^{\text {th }}$ of the wavelength for the frequency of interest (23). Most of the low frequency attenuation is a result of the expansion into the chamber. By placing the acoustic material in the cavity, the overall effective expansion area is reduced. As a result, there is a potential for a drop in TL at the low frequencies. These affects should be minimized because most of the acoustical energy exists in these frequencies. Zi derived properties for 
the complex acoustical impedance, $\bar{Z}$, and wave number, $\bar{k}$, of an element (5). These properties are primarily a function of density, $\rho_{o}$, and flow resistivity, $\sigma$, as seen in Equation 2-36 and Equation 2-37, respectively.

$$
\begin{aligned}
& \frac{\bar{z}}{z_{o}}=1.0+0.0954\left(\frac{\rho_{o} f}{\sigma}\right)^{-0.754}-j 0.085\left(\frac{\rho_{o} f}{\sigma}\right)^{-0.732} \quad \text { Equation 2-36 } \\
& \frac{\bar{k}}{k_{o}}=1.0+0.16\left(\frac{\rho_{o} f}{\sigma}\right)^{-0.577}-j 0.189\left(\frac{\rho_{o} f}{\sigma}\right)^{-0.595} \quad \text { Equation 2-37 }
\end{aligned}
$$

For the FEM software packages, such as LMS's Virtual Lab, when modeling absorptive materials the input variables are the flow resistivity, porosity, and tortuosity, as well as the properties of the medium $c$ and $\rho_{o}$. If the sound absorption properties were measured experimentally, it is typically done with the two-cavity method. The flow resistivity can be back calculated from Equation 2-36. Lord et al. has displayed that flow resistivity is primarily a function of the material density and inversely proportional to the thickness (23). Also, all glassfiber materials have roughly the same flow resistivity values relative to their density. Therefore, Lord et al. demonstrated that the flow resistivity can be estimated for glass-fiber materials and produce accurate results regardless if the true values are nearly double or half that of which was estimated (23). It will be shown later in this thesis that the above statement holds true when the author conducts a parametric study by varying flow resistivity properties of glass-fiber materials resulting in insignificant differences in TL. Because of this, it was chosen to only estimate the material properties rather than to perform sound absorption measurements for the experimental models used in this thesis. This proved to be a viable option. 


\subsection{Additional effects on muffler performance}

Noise radiated from an exhaust system contains three components. The major component is the noise generated by the pulsated pressure waves due to the periodic combustion process. The second component is the flow noise created by the turbulent exhaust gases. Lastly, the structural noises that become excited due to the previous two noise sources (2). These noise sources can be altered depending on the operating and loading conditions of the engine. Some of the most significant influences on noise radiated from an exhaust system that are influenced by the loading and operating conditions, will be discussed in this section.

\subsubsection{Temperature effects}

By varying the temperature inside the muffler, the overall performance of the system can be drastically altered. This temperature effect is one of the most important parameters to be considered when determining how a muffler system will perform. The driving parameters that temperature will affect are the speed at which the acoustic waves propagate and the density of the medium. The density of the medium can be calculated while taking into account the moisture in the air and assuming the ideal gas law (incompressible medium). This is shown in Equation $2-38$ (25). In the equation, $P_{\text {vapor, sat. }}$ is the $100 \%$ saturated pressure of moist air, the vapor pressure, $P_{\text {vapor }}$, can be calculated from the two variables, saturated pressure and relative humidity $R H . R_{m i x}$ is the mixture ratio of vapor pressure to the overall pressure. For engines running at very high speeds or at locations with tight bends there is a likely chance that compressible flow will exist. In this case, an empirical formula for calculation of density would have to be used. These effects have been neglected in this thesis as the compressible flow only occurs at a 
few locations, on average the exhaust system can be considered incompressible. It can also be shown that the speed of sound has more influence on the TL curve than the density of the medium. In Keske's dissertation it is stated that the change in density does not affect muffler performance because uniform tubes are a function of the specific acoustic impedance of the tube, $Y_{o}(6)$. This is indeed true for uniform tubes but does not hold true for lumped shunt and lumped in-line elements. These elements make up a significant portion of the elements inside of the muffler. Hence, a change in density of the medium should not be ignored when determining muffler performance.

$$
\rho_{o}\left[\frac{\mathrm{kg}}{\mathrm{m}^{3}}\right]=\frac{P_{S}[\mathrm{kPa}]\left(1+R_{\operatorname{mix}}\right)}{0.28703(T[C]+273.15)\left(1+1.16078 \cdot R_{\operatorname{mix}}\right)}
$$

where:

$$
\begin{gathered}
R_{\text {mix }}=0.622 \frac{P_{\text {vapor }}}{P_{S}-P_{\text {vapor }}} \\
P_{\text {vapor }}=\frac{R H \cdot P_{\text {vapor,sat. }}}{100} \\
P_{\text {vapor, sat. }}=0.61121 \cdot e^{\frac{17.67 \cdot T}{T+243.5}}
\end{gathered}
$$

As mentioned previously, the variable that is the most influenced by a changing temperature of an exhaust system, is the speed of wave propagation in the medium. Keske performed a full chemical analysis between the exhaust gas of a gasoline engine and the atmosphere (6). It was determined that given the same temperature and density, the speed of propagation through the two mediums is within $2 \%$ of one another. Based on this, the calculation for the speed of sound in air will be used for this thesis. The speed of sound is calculated based on Equation 2-39 and is solely a function of temperature (6).

$$
c(T)=\sqrt{\frac{\gamma(T) \cdot R \cdot T}{M}}
$$


In Equation 2-39, $\gamma$ represents the adiabatic index number which is the ratio of the specific heats of the medium. This value is typically assumed to be $1.40 \mathrm{but}$ does vary slightly with temperature, therefore the temperature effects should not be ignored (6). $R$ is the molar gas constant $\left(8.31451 \frac{\mathrm{J}}{\mathrm{mol} \cdot \mathrm{K}}\right)$ and $M_{\text {air }}$ is the mean molar mass of air $\left(0.0289645 \frac{\mathrm{kg}}{\mathrm{mol}}\right)$. Both are constant as a function of temperature. An empirical formula was derived for the speed of sound to only include the variable $T$ and can be seen in Equation 2-40 (31).

$$
c[\mathrm{~m} / \mathrm{s}]=\left(331.4+0.606 \cdot T\left[{ }^{\circ} \mathrm{C}\right]\right) \quad \text { Equation } 2-40
$$

Typically when transmission loss measurements are made, the ambient conditions of the air have a speed of sound around $345 \mathrm{~m} / \mathrm{s}$. On the other hand, in actual operation the exhaust system can see temperatures well in excess of $500{ }^{\circ} \mathrm{C}$. The increase in temperature causes the speed of sound to increase beyond $630 \mathrm{~m} /$ $s$, nearly double the speed of sound in ambient conditions. It is crucial to both understand how these parameters change the Transmission Loss of the muffler as well as what the actual operating conditions of the exhaust system are.

Lord et al. described that when the temperature inside of the exhaust system is changed from $T_{1}$ to $T_{2}$, there is a proportional frequency shift as shown in Equation 2-41 (23). The author has investigated this claim and has achieved the same results when a constant temperature is applied across the entire exhaust system. A sample Transmission Loss was calculated for an 8inch diameter cylindrical muffler using the transfer matrix method. This was done assuming plane waves through the entire system and elevating the temperature inside the muffler. The results are shown in Figure 2.11. It will be shown later that these results were achieved numerically as well in Virtual.Lab and the results correlate well in the plane wave region of the muffler. 


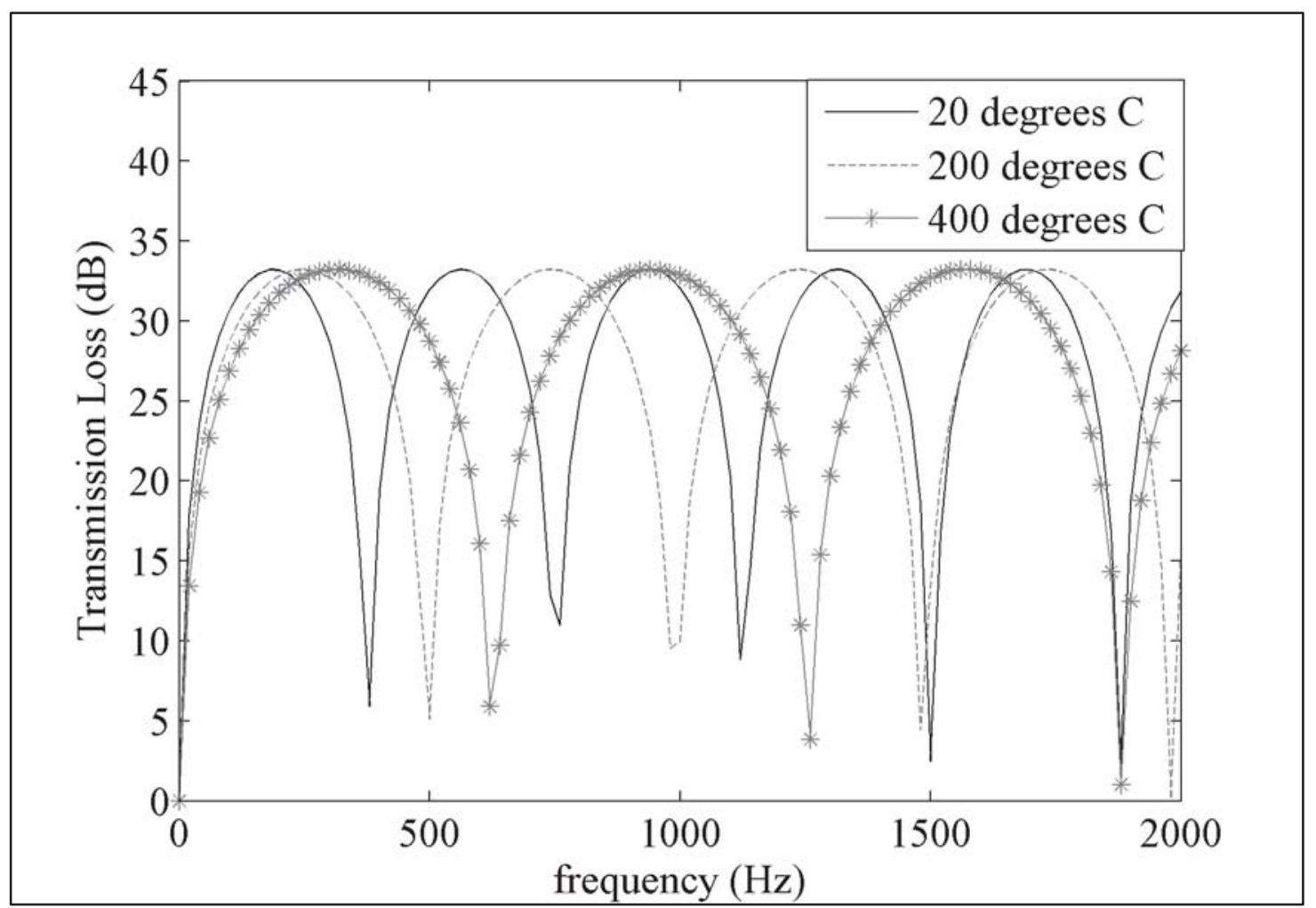

Figure 2.11: Transmission Loss of a cylindrical muffler when the temperature of the medium is varied.

$$
f_{2}=f_{1} \frac{\sqrt{T_{1}+\left(T_{2}-T_{1}\right)}}{\sqrt{T_{1}}}
$$

Equation 2-41

As can be seen in the figure, there is a large frequency shift in transmission loss when elevated temperatures are considered. The point to make here is that this is the ideal case where there is no temperature gradient inside the exhaust system. This is not the case in actual operation, expecially when large expansions and absorptive material are considered. The author of this thesis has performed work with predicting transmission loss of an exhaust system under actual operating conditions. The temperature data was gathered experimentally for various sections of the muffler and then the temperature profiles were applied to blocks in the simulation model. Based on the experimental data aquired there was significant 
temperature differences between blocks (over $300^{\circ} \mathrm{C}$ between sections). The highest temperatures were in areas with the smallest cross-sectional area, and vice versa. Also, areas that had absorptive materials had some of the lowest temperatures in the exhaust system. After conducting the analysis, there was a clear frequency shift as shown in Figure 2.11. The difference, however, was that the general shape of the transmission loss was altered significantly in a random fashion. Although, overall the amplitudes of the transmission loss curve remained fairly constant. The difficult situation occurs when attempting to predict the overall sound pressure level of the exhaust system while the engine is running because only specific frequencies are of concern. In order to get accurate TL amplitudes at these frequencies, precise temperature profiles are crucial.

Usually the muffler prototype is not built until after the simulations are completed. Therefore, gathering experimental data for temperature profiles is not feasible. The other method is to perform a coupled engine-muffler CFD analysis to determine the flow and temperature profiles in the exhaust system. The results can then be coupled to the FEM simulation. The advantage is the temperature profile will be determined for every nodal point, whereas experimentally this is not practical because the data can only be measured by blocks. The disadvantage is that the model is a prediction and can lead to major errors.

\subsubsection{Mean flow effects}

When exhaust gases are forced downstream in the exhaust system, the acoustical state variables are affected as shown in Equation 2-42 and Equation 2-43. The variable, $M$, is the Mach number and represents the flow velocity, $\bar{V}$, divided by $c$ (2). As shown in the equations, the "progressive" forward traveling wave propagates at a speed of $c+\bar{V}$ while the "reflective" backward traveling 
waves propagation speed is reduced to $c-\bar{V}$. Due to the change in the state variables, it is expected that the behavior of TL will change based on the mean flow rate.

$$
\begin{array}{cc}
p(x, t)=\left(C_{1} e^{\left(\frac{-j k_{0} x}{1+M}\right)}+C_{2} e^{\left(\frac{+j k_{0} x}{1-M}\right)}\right) e^{j \omega t} & \text { Equation 2-42 } \\
v(x, t)=\frac{1}{Y_{o}}\left(C_{1} e^{\left(\frac{-j k_{0} x}{1+M}\right)}-C_{2} e^{\left(\frac{+j k_{0} x}{1-M}\right)}\right) e^{j \omega t} & \text { Equation 2-43 }
\end{array}
$$

Munjal describes that with the addition of mean flow the real (resistive) part of the muffler impedance is reduced whereas the imaginary (reactive) part is relatively unaffected (2). It is expected that because of this, there is an overall decrease in TL as a function of flow velocity (2). FEM simulation work done in this thesis incorporated mass flow and the findings showed an overall drop in TL as a function of $M a$. Although, the general shape of the TL was relatively unchanged. This is not the case for dissipative ducts however as the attenuation properties of the acoustical material are shifted to a higher frequency in the presence of mean flow (2). This, in turn, causes an increase in TL at the higher frequencies while reducing the TL in the lower frequency spectrum. In many mass flow rate investigations conducted, it has been very difficult to determine a trend pattern for the effects on mass flow rate inside of a muffler. Montenegro et al. and Ji et al. saw substantial increases in TL with an increase in mass flow rate, each of which had a substantial amount of perforated tubing in the muffler designs (26, 12). Many other results from researchers showed a general flattening of TL, where all the peaks and troughs were almost completely removed and there was a general decrease in TL $(17,13,27)$. 


\subsubsection{Flow noise generation}

The other important component of the overall noise radiated into the atmosphere, besides the pulsating sound waves created by the engine, is the noise generated due the turbulent exhaust gas (21). When trying to predict the flow noise generated, a difficulty that arises is due to the random broadband behavior of the noise created. Empirical formulas have been created to provide a best fit solution to the problem such as the one created by Wiemeler et al. which will be described in this section (21).

Sound sources can be described as either monopole, dipole, or quadrupole which is depends on the order of the source (21). A monopole source can be defined as a source radiating out in the zeroth order, representing a spherical radiation pattern that would typically show a harmonic character (23). A dipole source is a source radiating in the first order. This source represents two pulsating spheres out of phase with one another that causes local forces propagating in a direction in line with the two local spheres (23). The dipole sources do not have a harmonic character to them and will have many local variations which will not coincide with forces in other locations. A quadrupole source is a source radiating in the second order and is typically seen in turbulence noise in free jets (21). The ability for these sources to be converted to flow noise is determined by the noise generation efficiency, $\eta_{\text {noise }}$. This parameter is dependent on the type of source $S_{t}$, the Mach number Ma, and the noise conversion constant $K_{n}$ which is shown in Equation 2-44 (21). 


$$
\eta_{\text {noise }}=\frac{\text { Sound Power }}{\text { Flow Power }}=K_{n} \cdot(M a)^{S_{t}} \quad \text { Equation 2-44 }
$$

where:

$$
\begin{array}{ll}
\text { Monopole Sources } & \sim S_{t}=1 \\
\text { Dipole Sources } & \sim S_{t}=2 \\
\text { Quadrupole Sources } & \sim S_{t}=4
\end{array}
$$

The conversion efficiency for monopole sources is linear in characteristic, dipole sources are exponential, and quadrupole sources are to the power of four. In order to prevent significant flow noise, higher order sources should be avoided. Unfortunately, through the works of Wiemeler et al., dipole sources are dominate for most exhaust system applications, especially in high performance engines which see high flow rates (21). The noise conversion constant $K_{n}$ is an unknown variable in the equation and is a function of the muffler geometry.

The only way to determine the variables present in the exhaust system, seen in Equation 2-44, is experimentally. This is completed with the use of a linear level notation, as shown in Equation 2-45. For this case, flow noise measurements are made in the free field to determine the sound pressure level versus Mach number; where Mach is plotted on a logarithmic x-axis. This can be very useful to describe what types of sources dominate in the flow stream as well. The type of the source, $S_{t}$, present in the flow stream is related to the slope of the line as demonstrated in Equation 2-46. It is noted that dipole sources dominate in the majority of all muffler configurations, therefore the second term in Equation 2-45 is approximately the same for all designs (21). The difference lie in the first term which, comparing different configurations, becomes mainly dependent on the noise conversion constant, $K_{n}$. 


$$
\begin{array}{cc}
S P L=f c n\left(\eta_{\text {noise }}\right)+10\left(S_{t}+3\right) \log _{10}(M a) & \text { Equation 2-45 } \\
S_{t}=(\text { slope }-3) / 10 & \text { Equation 2-46 }
\end{array}
$$

\subsection{Muffler impedance}

Many items can be extracted from the 4-pole parameters of a muffler. One item which can be calculated is the muffler impedance. The muffler impedance is of particular importance when determining source properties as the inlet velocity is also a function of the muffler impedance. The relation between the muffler impedance and the 4-pole parameters is shown in Equation 2-47 (15). The real part of the impedance represents the resistivity (the resistance the acoustical pressure undergoes when passing through the muffler) and should always be a positive value. The imaginary part is the reactance and describes how well the acoustical energy is stored in the muffler (15). Usually a high imaginary part of the muffler impedance signifies highly dissipative muffler. As stated previously, the muffler impedance will be used to determine inlet velocity which in turn will be used to predict sound pressure levels in the atmosphere of the entire exhaust system, including the source.

$$
Z_{m}=\left(\frac{B}{C}\right)^{\frac{1}{2}}
$$




\subsection{Implementation of Source Data}

Now that Transmission Loss in ambient conditions can be accurately predicted for nearly all acoustic filters, one of the driving research areas is the prediction of sound pressure levels of the exhaust system in actual operating conditions. In order to accomplish this task, the source information needs to be incorporated into the simulation model. This section will only cover the theory in incorporating the source. The actual implementation into a FEM solver will be discussed in a later chapter.

Referring back to Figure 2.5 for the transmission network of an acoustic filter, a one port model for the source can be created as well, shown in Figure 2.12. The model assumes only plane waves exist in the exhaust pipe (20). The subscript $i$ represents an integer from 1 to $n$, the number of loads applied. Using electrical analogies, the diagram can be described as shown in Equation 2-48.

$$
P_{s} \cdot Z_{i}-P_{i} \cdot Z_{s}=P_{i} \cdot Z_{i}
$$

Equation 2-48

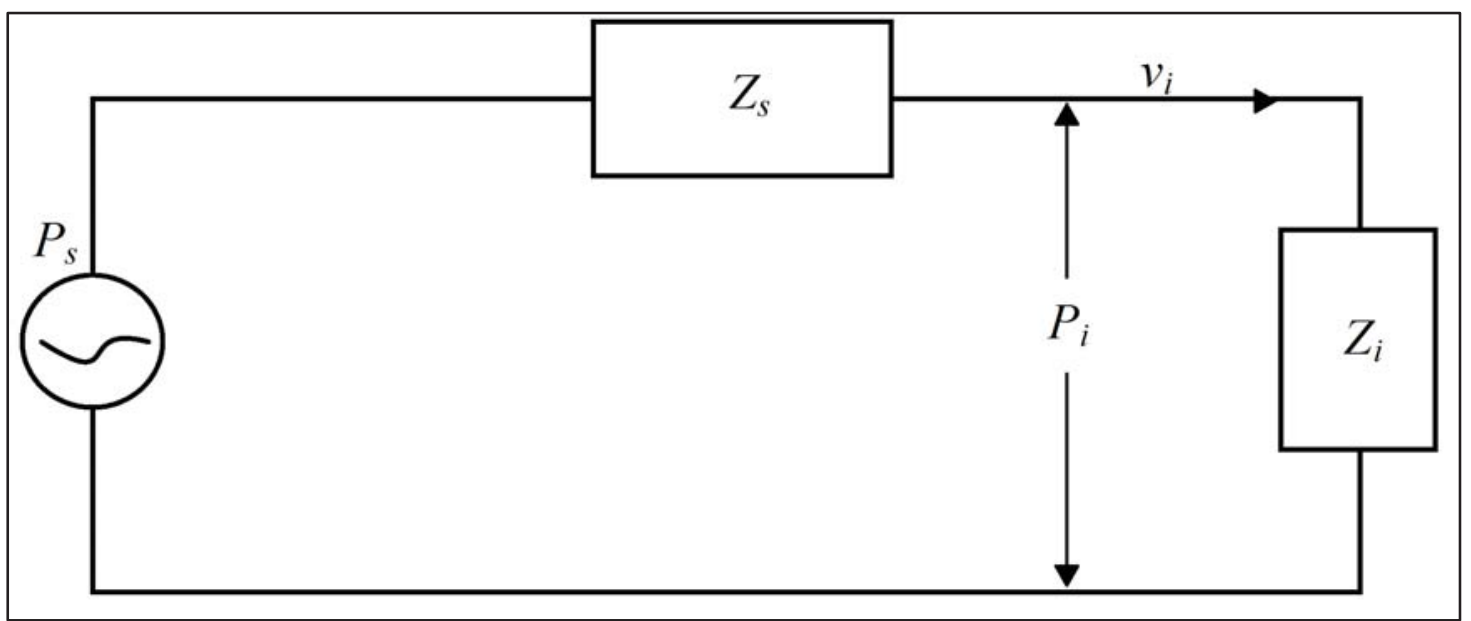

Figure 2.12: One port model to determine source properties. 
A non-linear simulation package, such as "GT-Power", can be used to determine the unsteady 1-D gas flow leaving the exhaust pipe from the thermodynamic cycle as long as plane wave propagation exists. In Figure 2.12 the two unknown variables are the source properties, $P_{s}$ and $Z_{s}$. The known load, $Z_{i}$, is applied to the model and $P_{i}$ is measured. Using a non-linear solver, the known load can be applied as a straight pipe. By changing the pipe length (changing the applied load, $Z_{i}$ ) you are left with two equations and two unknowns. Performing this measurement twice with two different loading conditions, denoted 1 and 2 , the source impedance and source pressure can be characterized by Equation 2-49 and Equation 2-50, respectively (20). Typically the load is changed more than twice in order to gather an over-determined set of equations and increase the accuracy of the source data.

$$
\begin{aligned}
& Z_{s}=\frac{P_{1} Z_{1}-P_{2} Z_{1}}{\left(P_{2}\left(\frac{Z_{1}}{Z_{2}}\right)-P_{1}\right)} \\
& P_{S}=P_{2}\left(1+\frac{Z_{S}}{Z_{2}}\right)
\end{aligned}
$$

Equation 2-49

Equation 2-50

The downfall of implementing source information into a FEM simulation package, such as LMS's Virtual.Lab, is that a pressure cannot be applied directly as a boundary condition. Consequently, the boundary conditions need to be the source impedance and the input velocity. First, the impedance of the muffler system needs to be calculated using Equation 2-47. Referring back to Figure 2.12 and replacing $Z_{i}$ with the impedance of the muffler, $P_{i}$ can be calculated by rearranging Equation 2-50. The input velocity, $v$, can be described by the total system given Equation 2-51. Doing this type of process has proven to be very exhaustive as all of the source properties, 4-pole parameters, and muffler 
impedance are a function of the engine load and operating speed. In order to achieve the desired 4-pole parameters, the correct temperature profile is crucial in the accuracy of the source implementation. The author and his colleague have implemented this method into Virtual.Lab for a classified project and achieved fairly accurate correlation to experimental data.

$$
v=\frac{P_{s}}{Z_{s}+Z_{m}}
$$




\section{Chapter 3. Experimental Method}

In this chapter the underlying theory for the calculation of Transmission Loss will be established. Also, the incorporation of mass flow in the current stationary test step will be described and implemented. The experimental models used in this thesis will be discussed at the end of Chapter 4 and the results will be presented in Chapter 5.

\subsection{Transmission Loss measurement}

The Transmission Loss of a muffler can be calculated in a variety of ways, including the use of the decomposition method, two load method, and the two source-location method (28). The decomposition method requires two microphones at the inlet section of the pipe to decompose the progressive and reflective waves and a single microphone in the outlet section where only transmitted waves exist (28). To achieve only transmitted waves in the tailpipe (no reflective waves) then there must be an anechoic termination at the outlet. Although this is easily done in simulation, experimentally this is not feasible. As a result, this method will not be discussed further. The other two methods are the most practiced and will be discussed next.

\subsubsection{Two load method}

Because an anechoic termination cannot accurately be applied to the outlet of the muffler, different testing techniques such as two load method need to be practiced. The two-load method consists of four microphones. Two microphones are place both in the inlet piping and outlet piping to determine both the 
progressive and reflective waves. This technique is used to derive the 4-pole parameters of the muffler and then, using Equation 2-22, Transmission Loss can be calculated. As there are four unknowns (4-pole parameters) and only two equations relating the state variables (as seen in Equation 2-16), there must be two loading conditions. Typically this is done by leaving the termination open to the atmosphere and proceeded by closing the outlet tubing using a rigid termination, such as a steel plate. The diagram of the two test configurations are depicted in Figure 3.1.

In Figure 3.1, the numbers represent the channel number of the microphone at that specific location. Given the two test configurations, six total Frequency Response Functions (FRF) are calculated; where channel 3 is always referred to as the reference channel. The derivation of the 4-pole parameters was described in the works of Tao et al.; only the equations will be presented in this thesis (28). The calculation of the 4-pole parameters are shown in Equation 3-1through Equation

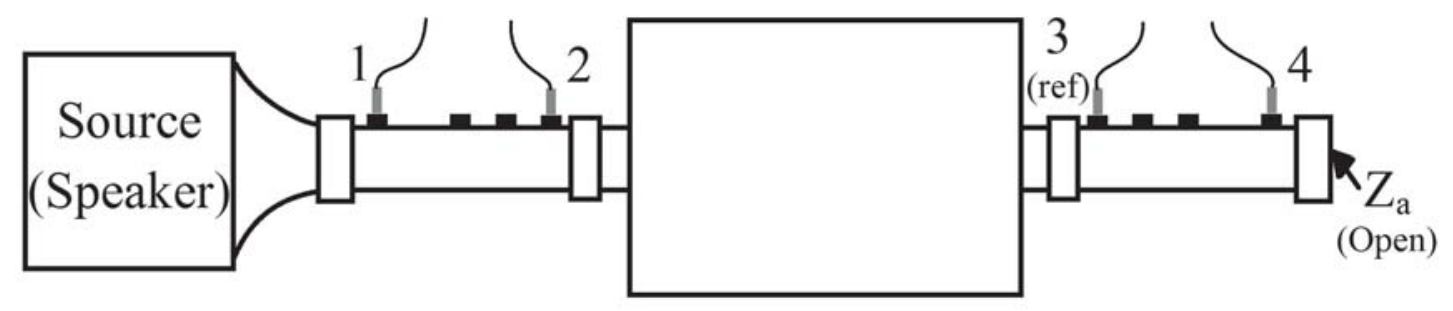

(a)

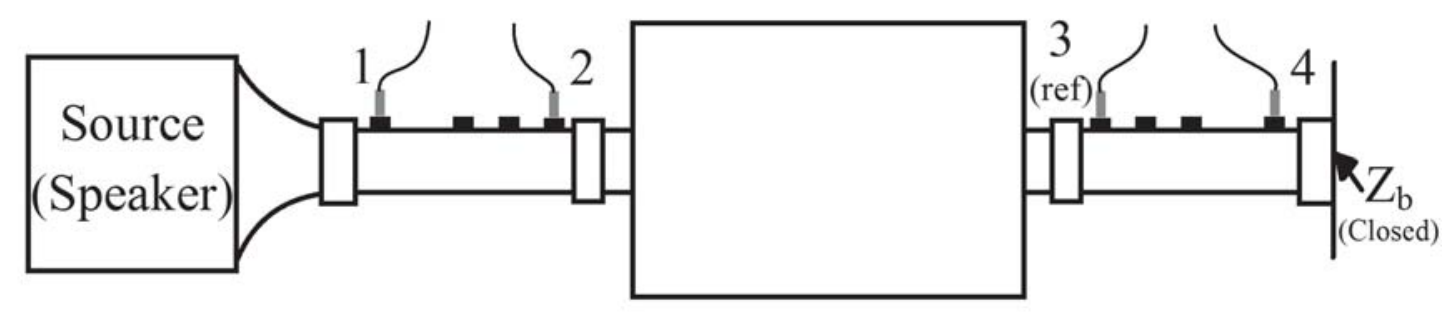

(b)

Figure 3.1: Two Load method in determining TL of muffler for (a) step 1 when outlet is open to atmosphere and (b) step 2 when load at outlet is rigidly closed. 
3-4.The variable, $\mathrm{H}$, represents an FRF with the first number designated as the output channel, the second number represents the reference channel (Ch. 3), and the letter represents the test configuration (28). The $\Delta$ symbol is the determinant of the transfer matrix, in which case, for a uniform tube would be equal to one.

$$
\begin{array}{cc}
A_{23}=\frac{\Delta_{34}\left(H_{23 a} H_{43 a}-H_{23 b} H_{43 a}\right)+D_{34}\left(H_{23 b}-H_{23 a}\right)}{\Delta_{34}\left(H_{43 b}-H_{43 a}\right)} & \text { Equation 3-1 } \\
B_{23}=\frac{B_{34}\left(H_{23 a}-H_{23 b}\right)}{\Delta_{34}\left(H_{43 b}-H_{43 a}\right)} & \text { Equation 3-2 } \\
C_{23}=\frac{\left(H_{13 a}-A_{12} H_{23 a}\right)\left(\Delta_{34} H_{43 b}-D_{34}\right)}{B_{12} \Delta_{34}\left(H_{43 b}-H_{43 a}\right)} & \text { Equation 3-3 } \\
-\frac{\left(H_{13 b}-A_{12} H_{23 b}\right)\left(\Delta_{34} H_{43 a}-D_{34}\right)}{B_{12} \Delta_{34}\left(H_{43 b}-H_{43 a}\right)} & \\
D_{23}=\frac{\Delta_{34}\left(H_{23 a} H_{43 a}-H_{23 b} H_{43 a}\right)+D_{34}\left(H_{23 b}-H_{23 a}\right)}{\Delta_{34}\left(H_{43 b}-H_{43 a}\right)} & \text { Equation 3-4 }
\end{array}
$$

As presented in the equations above, the 4-pole parameters of the uniform tubes between microphones 1-2 and 3-4 must be known. They are calculated using Equation 2-18. Replacing the necessary variables for the pipe between microphones1-2, the 4-pole parameters are calculated and can be seen in Equation 3-5 (2). To calculate the 4-pole parameters between microphones 3-4 the same equation is used. Typically the diameters and lengths are identical, resulting in identical 4-pole parameters. Equation 3-5 only holds true for plane wave propagation, therefore, this method of TL testing is adequate in the frequency range given by Equation 2-27. 


$$
\left[\begin{array}{ll}
A_{12} & B_{12} \\
C_{12} & D_{12}
\end{array}\right]=\left[\begin{array}{cc}
\cos \left(\frac{2 \pi f L_{12}}{c}\right) & j \rho c \sin \left(\frac{2 \pi f L_{12}}{c}\right) \\
j \frac{1}{\rho c} \sin \left(\frac{2 \pi f L_{12}}{c}\right) & \cos \left(\frac{2 \pi f L_{12}}{c}\right)
\end{array}\right] \quad \text { Equation 3-5 }
$$

The downfall with using the two-location method is if there are not substantial differences between the two loads attached at the termination, then the equation for TL breaks down (6). It might be assumed that by closing the outlet, the load is sufficiently changed. As the load is a function of frequency, this has been shown to not always be the case, particularly at the lower frequencies. A much more viable and accurate method is the two source-location method which is not limited to these shortcomings and will be discussed next.

\subsubsection{Two source - location method}

The two source-location method is an improved method based on the twolocation method stated previously. In this method, the source is moved from the inlet to the outlet location for the second configuration. Because of the two different testing configurations, the terminations do not need to be identical nor anechoic. Typically, however, you would want to minimize the background noise measured. Due to this, it would be advantageous to place some absorptive material at the end of the outlet/inlet.

Since this technique is the most accurate and stable measurement, it was implemented throughout the duration of the research project. This technique found another benefit when incorporating mass flow because air was free to leave the tailpipe. This could not be the case for the two-load configuration if the outlet was closed. A diagram of the two test configurations is shown in Figure 3.2. All of the 


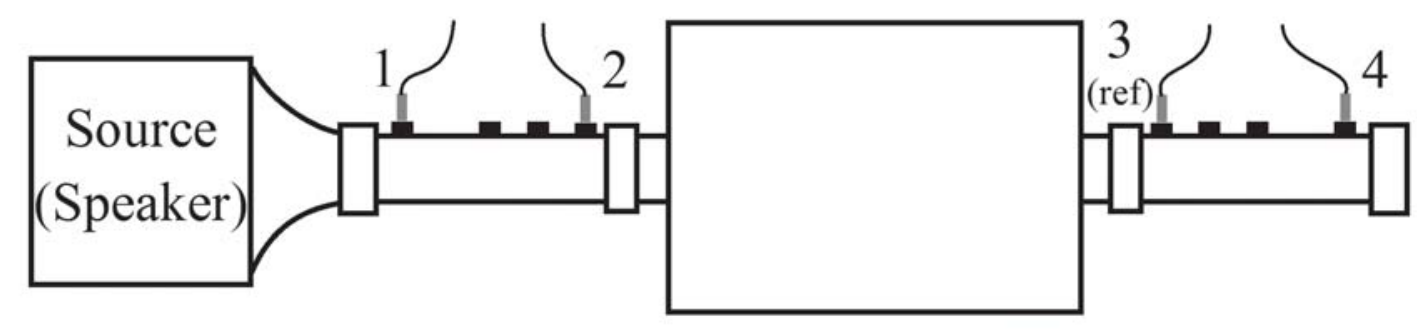

(a)

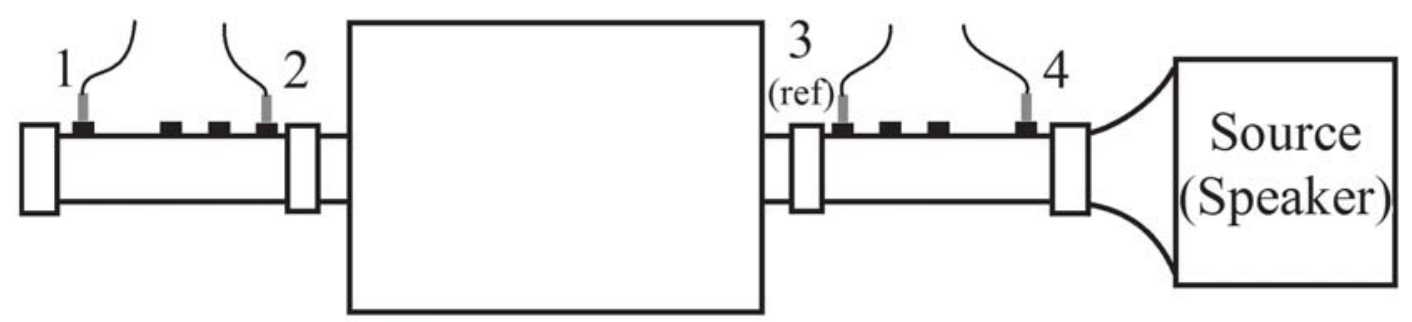

(b)

Figure 3.2: Two Source-Location method in determining TL of muffler for (a) step 1 when source is at inlet and (b) step 2 when source is at outlet.

previous equations presented in Section 3.1.1 are used in the calculation of TL and the nomenclature remains the same.

\subsection{Test Setup}

The initial test setup was developed by Keske during the process of completing his dissertation (6). The initial test setup is shown in Figure 1.2 and can be used for either the two-load or two source-location method to determine TL. The latter of the two was practiced. Keske fabricated TL test tubes and adapters for the microphones so that $1 / 4$ " ICP microphones with $1 / 2$ " preamplifiers could be used (6). The diaphragm of the microphone is placed flush with the inside of the test tube. Through personal experience, if the diaphragm of the microphone was not placed exactly flush with the tube, there would be distortion 
of the TL curve. This was a result of the microphone causing interferences with the plane waves in the tube which, in turn, caused unwanted reflections.

The TL test tubes fabricated can be used for variable microphone spacings of 35, 70, 105, and $140 \mathrm{~mm}$. From experimentation conducted in this thesis, there is a few things to conclude about the microphone spacing. The first is that typically larger microphone spacings will more accurately calculate lower frequency TL due to the large wavelengths. The shorter microphone spacings will more accurately calculate the higher frequency TL. The second item noticed with the microphone spacing is near locations where the calculation of the progressive wave breaks down. At these locations the TL takes on the appearance of an acoustical resonance. The calculation for the progressive wave is shown in Equation 3-6, where 1 and 2 represents microphones 1 and 2 (6). The equation breaks down when the $\sin \left(k l_{12}\right)$ goes to zero. The frequencies when this occurs can be calculated using Equation 3-7 and is shown for the four microphone spacings in Table 3.1. The effects are apparent over a fairly large frequency range centralized at the frequencies presented in the table. The frequency-microphone spacing combination should be avoided for accurate results.

During the completion of this thesis, the researcher had all of the microphone spacings measured simultaneously; the best spacing was kept based on frequency. This showed to greatly shorten the TL testing time since there was no need to continuously change microphone spacings. Being able to do this is dependent on the number of Data Acquisition (DAQ) channels and instrumentation available. It should be noted, however, that although the microphone spacing can change between measurements, the location of microphones 2 and 3 should not change as this would change the acoustical properties of the muffler. 
Table 3.1

The frequencies $(\mathrm{Hz})$ when the transmission loss calculation breaks down when $c=348 \frac{\mathrm{m}}{\mathrm{s}}$.

\begin{tabular}{|c|c|c|c|c|}
\hline & \multicolumn{4}{|c|}{ Length Between Microphones (mm) } \\
\hline $\mathbf{n}$ & $\mathbf{3 5}$ & $\mathbf{7 0}$ & $\mathbf{1 0 5}$ & $\mathbf{1 4 0}$ \\
\hline $\mathbf{1}$ & 4946 & 2473 & 1649 & 1237 \\
\hline $\mathbf{2}$ & 9893 & 4946 & 3298 & 2473 \\
\hline $\mathbf{3}$ & 14839 & 7420 & 4946 & 3710 \\
\hline $\mathbf{4}$ & 19786 & 9893 & 6595 & 4946 \\
\hline $\mathbf{5}$ & 24732 & 12366 & 8244 & 6183 \\
\hline
\end{tabular}

$$
\begin{array}{cc}
p_{i}=\frac{1}{2 j \sin \left(k l_{12}\right)}\left[p_{1}(j \omega) e^{j k z_{2}}-p_{2}(j \omega) e^{\left(j k z_{1}\right)}\right] & \text { Equation 3-6 } \\
f_{\text {res }}=\frac{n c}{2 L_{12}} \quad \text { for } n=1,2,3, \ldots & \text { Equation 3-7 }
\end{array}
$$

\subsubsection{Incorporating mass flow}

One of the goals of this thesis was to incorporate mass flow into the stationary test setup. The flow bench can be used for three primary functions: to determine how mass flow rates affect the transmission loss for various elements, to determine how much flow noise is generated, and to potentially be able to measure back pressure inside of the exhaust system.

In order to build the flow bench, a few items needed to be purchased. To be able to measure the flow an air velocity transducer was needed. The transducer purchased could measure air speeds up to $50.8 \mathrm{~m} / \mathrm{s}$ (about $0.15 \mathrm{Ma}$ ). Typically, some higher running engines will exceed this speed so it was necessary to upsize the pipe to a larger diameter and then apply a correction factor (the ratio between the two cross-sectional areas). The larger diameter pipe also was used to reduce 
the turbulence in the flow near the flow meter, resulting in a more accurate reading. The extended pipe was sufficiently long as to allow at least 10 pipe diameters before the flow meter so an accurate measurement could be made.

A $2.5 \mathrm{Hp}$ shop vacuum was used as the air flow power supply. The benefit of this selection is that the source could be used in two configurations. The first configuration is when the source was placed at the outlet and used as a vacuum. In the second configuration, the source was moved to the inlet and was used as a blower to force the air through the exhaust system. The first configuration proved to provide substantially less flow noise which helped in gathering better TL measurements. A variable autotransformer needed to be purchased in order to change the amount of airflow the shop vacuum supplied. The test setup for the flow bench can be seen in Figure 3.3.

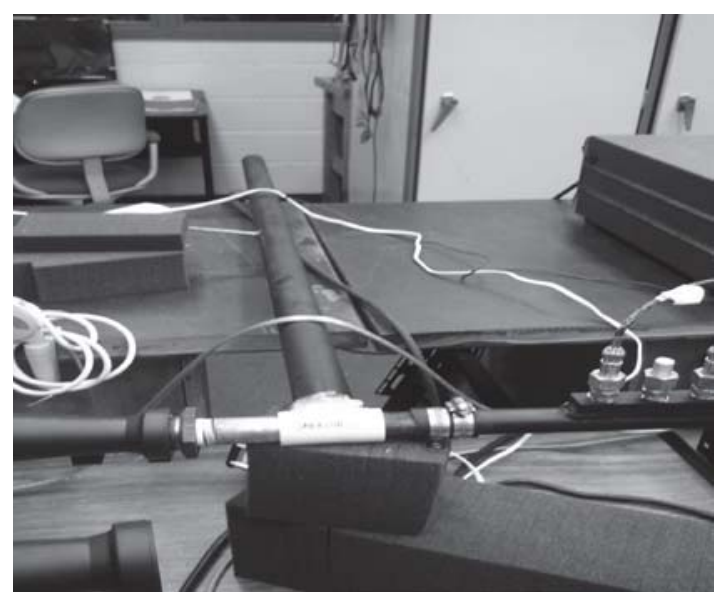

(a)

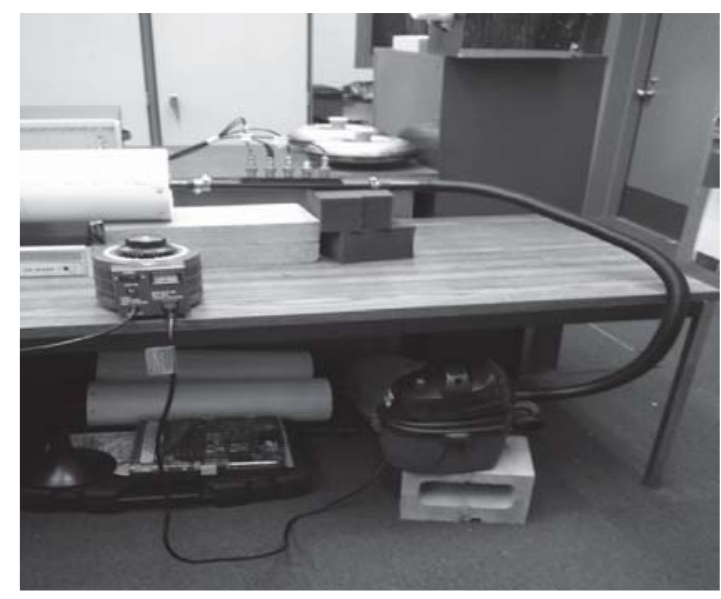

(b)

Figure 3.3: The developed flow bench showing (a) the flow meter and tube extension and (b) the air velocity source (Shop Vacuum) and controller (variable autotransformer). 


\subsection{Equipment selection and signal processing}

Typically the larger in diameter the speaker is, the lower in frequency the speaker can produce (as well as the lower the max frequency it can generate).

Because of this, Keske had two speakers incorporated in the test setup, a subwoofer which was used in the frequency range of $20-400 \mathrm{~Hz}$ and a significantly smaller speaker that was used in the frequency range of $400-3000 \mathrm{~Hz}$ (6). For the air flow testing, the smaller speaker did not have enough power to overcome the flow noise so a third speaker needed to be incorporated and a box was fabricated for it.

The dynamic range of the microphones proved to be the biggest issue with acquiring the FRF measurements, especially for the testing which included mean flow. This issue mainly arises because each microphone has their own FRF with respect to the sound source which are significantly different from one another. The different FRFs create difficulty when it is attempted to provide each channel with sufficient amount of energy across the frequency range of interest. If there is only one constant random sound source across the entire range, typically the higher frequencies would not receive enough energy. To correct for this, the channels would overload due to too much energy provided to the lower frequency spectrum. To resolve this limitation, the frequency spectrum is broken into different test sections. The power level on the source was properly selected for each one. The downfall is that this drastically increased the testing time.

The 1/4" ICP microphones used in the experiment have a dynamic range of $122 \mathrm{~dB}\left(25.18 \mathrm{~Pa}_{r m s}\right)$ which is equivalent to 1.6 Volts. It can be very difficult to determine if the dynamic range has been exceeded as the DAQ's used typically can acquire a voltage signal of $\pm 5 \mathrm{~V}$ or more. Although the dynamic range of the microphones may be below this. In this case, there would be no warning displayed 
on the software interface. Additionally, the coherences achieved once the dynamic range is exceeded would seem normal unless the experimentalist is familiar with the equipment and understands the effect of dynamic range (6). Initially during stationary TL testing, the experimentalist had this issue as the source was producing too much energy at particular frequencies. After troubleshooting, the root cause was determined. It will be shown in Section 3.3.2 that the dynamic range has been exceeded in a few frequencies when measuring TL on the flow bench. In that particular situation, the experimentalist felt it was necessary to exceed the dynamic range to sufficiently overcome the noise generated by flow. As expected, the TL at these particular locations was affected.

\subsubsection{Stationary TL testing}

When performing the TL testing without incorporating flow, the test setup should be as shown in Figure 1.1. The most effective excitation method was found to be a burst random signal, however, a burst chirp signal can be fairly effective as well. This type of excitation method for measuring FRF's was done in LMS's Test.Lab Spectral Testing Rev. 11B. In order to minimize leakage, it is ideal to have the excitation be a completely observed transient. The source is turned off before the end of the measurement period and the signal should decay into the noise floor. Since the decay rate is a function of the absorption in the outlet, it is beneficial to place sound absorption material in the outlet. This will also help to reduce the noise floor, in turn, reducing the noise in your measurement. During testing, the author set the source at $90 \%$ burst time which proved to be adequate. When the source is completely observed and the noise floor is sufficiently low, no window (uniform window) can to be used in the Fast-Fourier Transform (FFT). If there is some background noise, it would be recommended to use a Hanning window. 
When selecting the proper sampling parameters, it was proved necessary to allow the source to be on for sufficient time. The cleanest measurements came when the measurement time was set to $1 \mathrm{~s}$ which is when the block size is the same as the sample rate. When calculating the FRFs, it is necessary to perform at least 60 averages for the cleanest results. For the testing completed in this thesis, there were 100 averages for every test configuration. The $H_{v}$ estimator was used for calculating the FRFs, however, $H_{1}$ would have been sufficient as well.

As with any FRF measurement, the coherence of the signals should be monitored. A low coherence is most likely the result of having not enough energy in the signals downstream of the muffler. This particular issue can be very hard to deal with, especially with mufflers with a very high transmission loss. If the front channels are nearly exceeding the dynamic range of the microphones and the back channels (after the muffler) do not have enough energy, the only way to fix this is to use a lower sensitivity microphone for the back two locations. This was seen in the last few control mufflers that the researcher tested as the mufflers provided very good attenuation.

In order to provide adequate sound power to the microphones for the testing in this thesis, the frequency spectrum was broken into three sections and then pieced together. The frequency ranges were: $20-400 \mathrm{~Hz}, 400-1600 \mathrm{~Hz}$, and $1600-$ $3000 \mathrm{~Hz}$. The first frequency block was measured using the large subwoofer and the other two were tested using the small speaker.

\subsubsection{Mass flow TL testing}

The test configuration with air flow incorporated in the TL testing is shown in Figure 3.5. For pictures of the additional items that were added to the stationary 
test bench already in place, refer to Figure 3.3. Before collecting the FRF's for the models incorporating mass flow, it is important to first determine how much noise is being created by the turbulent flow. This is necessary to confirm that the source (speaker) is producing enough energy as to overcome the flow noise and to be the dominate noise contributor at every frequency spacing. The design had the potential to have the air flow upstream (used as a blower) as well as downstream (used as a vacuum). These two techniques needed to be compared. After performing this testing, it was clear that for all running speeds the vacuum technique was both quieter and smoother as compared to its counterpart. The results can be seen in Figure 3.4. Because noise from the flow was a concern during the TL testing, the vacuum configuration was determined to be the most appropriate.

A speaker volume of $10 \mathrm{~dB}$ higher than the flow noise at each frequency would typically be ideal. This proved to be a very daunting task for a number of reasons. One difficulty that arose is that the flow noise is loudest after passing through elements that cause disturbances in the flow pattern. Hence, the flow noise was significantly louder downstream of the muffler rather than upstream. This presents a problem as the speaker is upstream and by the time the source energy reaches downstream it is attenuated significantly. In order to provide the back channels with enough energy to overcome the flow noise the situation then arises about how to stay under the dynamic range of the front microphones as well as the overall maximum power of the source. The front microphones already have the lowest sensitivity of the microphones available. Simply changing the microphones would not resolve the issue. The new speaker that was used for this testing was either at or near maximum power output for the entire frequency span. Therefore, if a larger source would have been available, better measurements could have been taken. If the source had adequate power, the only limiting factor would be the dynamic range of the microphones. 


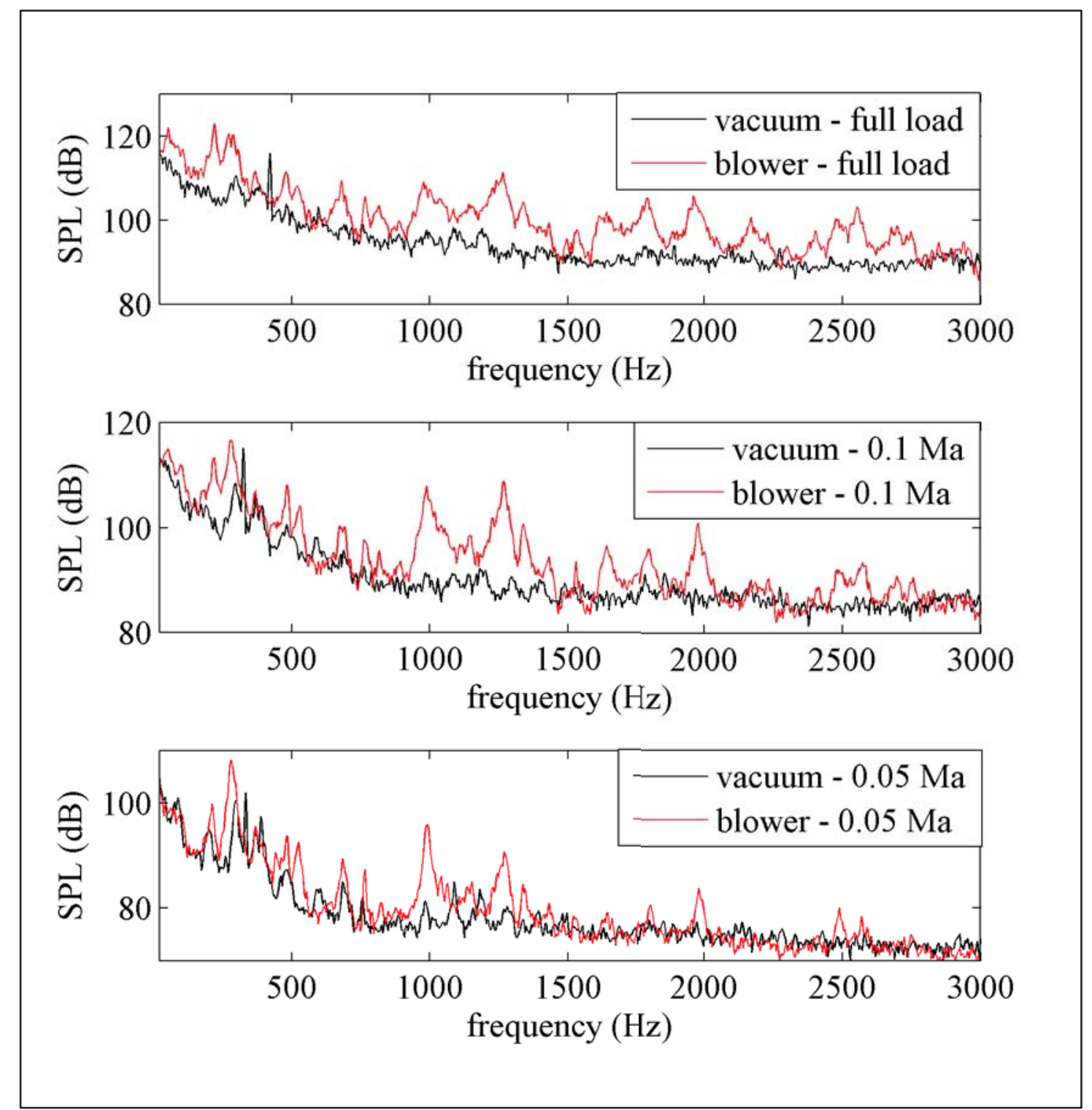

Figure 3.4: Flow noise spectrums inside of the TL test tube at microphone 3 (reference channel). The speed of sound was taken as $348 \mathrm{~m} / \mathrm{s}$. 
As stated previously one of the biggest challenges was to produce a signal that would be substantially louder than the noise generated by flow. The researcher first examined the possibility of using a burst random signal as done with the stationary testing and then breaking the frequency spectrum into four test segments. A signal was produced that was on the verge of going over the dynamic range of the microphones for each section. The results were not very promising as the signals on the back two microphones were only louder than .05Ma flow at very few frequencies (around half of the spectrum). When the flow was increased further, nearly the entire spectrum was less than the flow noise. The researcher knew that to truly see the effects of flow, higher speeds need to be seen. This method would not be sufficient to achieve desired results.

The method that was finally selected was to use a Stepped-Sine approach implemented in LMS's Test.Lab Rev.11B. This approach uses a closed-loop control algorithm to produce the FRF frequency line by frequency line. This has the potential to create the max signal output at every frequency line which is governed by the power available by the source and the dynamic range of the front

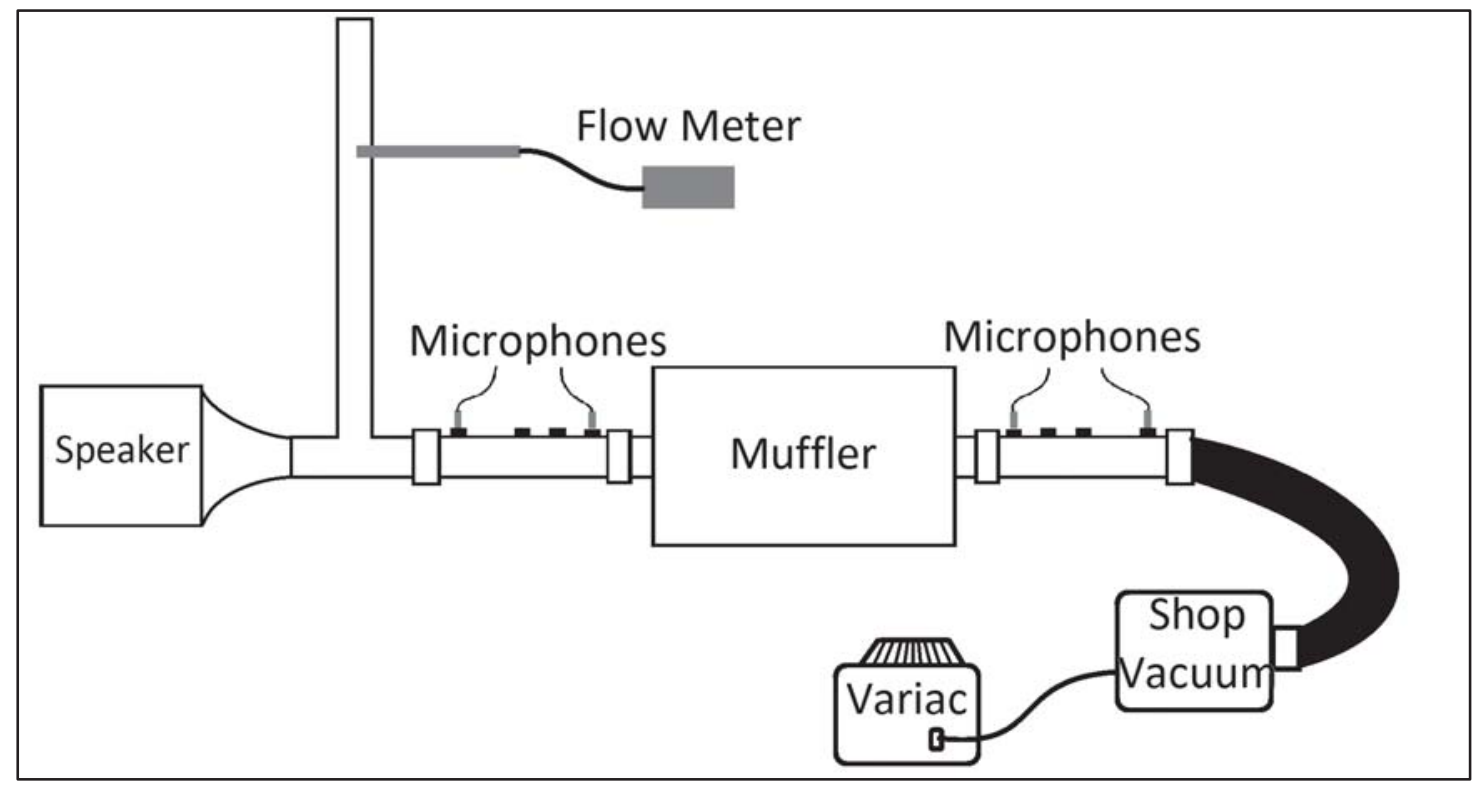

Figure 3.5: The flow bench test configuration for Transmission Loss testing. 
two microphones. The most time consuming part of this process is the creation of the control algorithm. The first step is to declare what your control channel is going to be. Because the goal is to maximize the dynamic range (or speaker volume), microphone 2 was initially selected (the one just before the inlet of the muffler). When writing the control algorithm, however, it was noticed that there were harmonic frequencies within the frequency spectrum. The harmonic frequencies caused the reference channel sound pressure signal to drop off compared to the microphone just upstream. This was the result of a standing wave (acoustical resonance) that was governed by the length of the pipe from the microphone to the inlet of the muffler. Because of this, it was necessary to write a control algorithm for the upstream microphone signal as well and then assemble all the frequency components together. Consequently, there were a total of fourteen test segments that needed to be assembled for the complete TL measurement.

After writing the control algorithms, the response spectrums were compared at microphone 3 located downstream of the muffler and shown in Figure 3.6. The Stepped Sine method is the far superior method for this type of application. Seen in the figure, there are instances when the stepped sine approach exceeds the dynamic range of the microphone. Again, there is signal distortion due to exceeding the dynamic range of the microphone. It should also be pointed out that this is the point downstream of the muffler. Upstream, the sound pressure level from the source is significantly larger and the flow generated noise is smaller. With this test setup, it was deemed necessary to exceed the dynamic range of the microphones in order to provide adequate power to all the channels. 
The difficulty with the Stepped Sine approach is because it is a closed loop approach. At any frequency line, if the flow noise is contributing to the overall noise then the control algorithm has the tendency to crash. This is because the software determines FRFs between the speaker and the control channel beforehand without the flow present. This FRF, along with the algorithm of the control channel, governs the initial output voltage of the speaker. In actual operation, the FRF between the source and microphone could be significantly different depending on the flow noise contribution. This proved to be a very difficult and

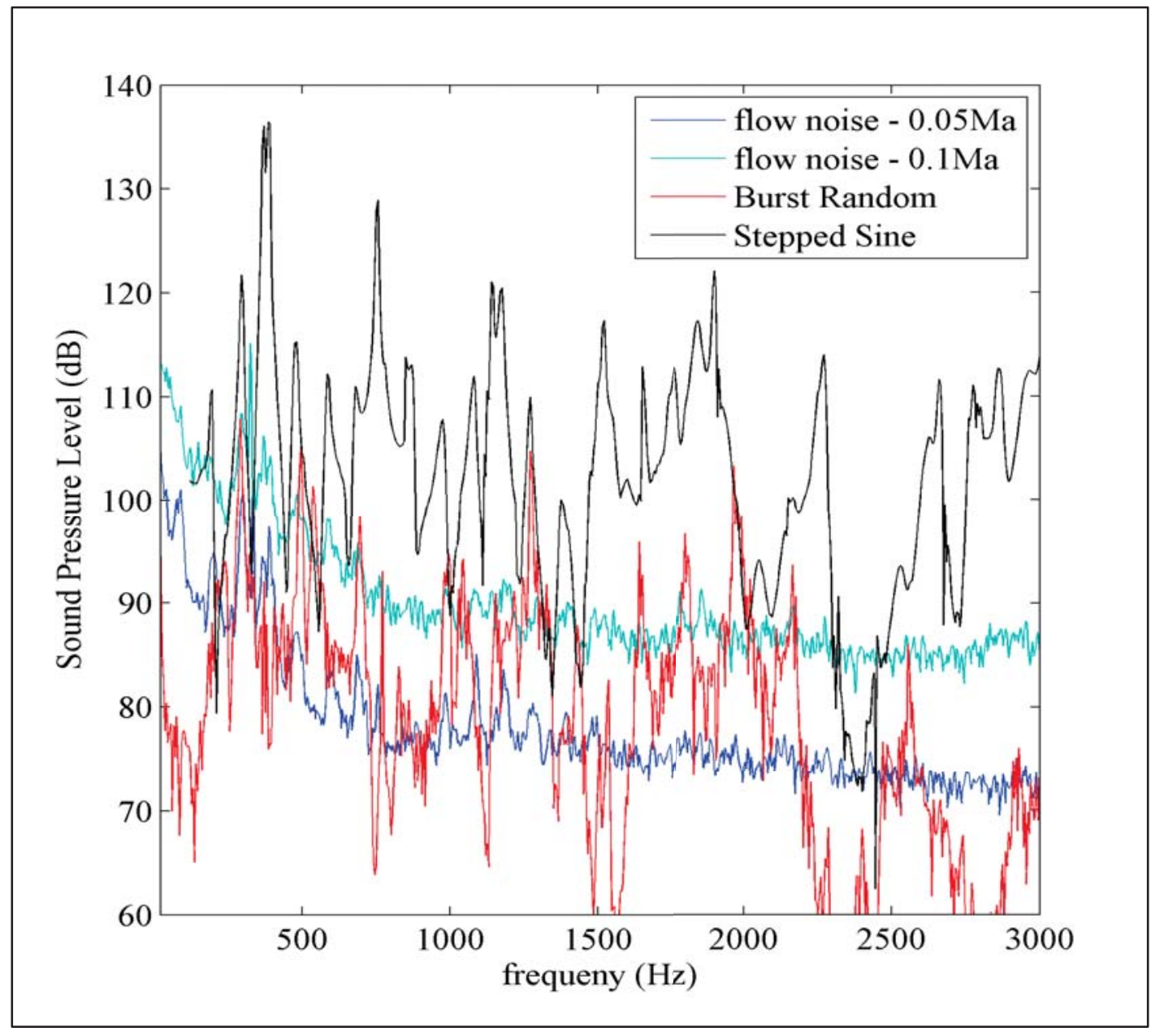

Figure 3.6: A comparison of different FRF measurement methods compared to the noise generated from turbulent flow. 
time consuming process, especially for higher air flow velocities because the control algorithm boundaries needed to be adjusted manually to provide sufficient results. The results in Figure 3.6 show that it is feasible to measure the TL of the muffler with flow speeds up to around $0.1 \mathrm{Ma}$ although some signal distortion is expected. Overall, the complete testing of two mufflers for varying flow levels of $0,0.05$, and $0.1 \mathrm{Ma}$ were measured which took roughly twenty hours to complete.

\subsection{Experimental Muffler testing}

The experimental testing of the mufflers was performed mainly to build confidence in the simulation work done for this thesis. The biggest portion of the research for this thesis was developing muffler models in LMS's Virtual.Lab and completing parametric studies on different elements to see their effects on muffler performance. The experimental portion of this research served as a correlation method to the simulation models and to give the researcher valuable experience in taking good data and understanding equipment limitations.

Keske had a few control mufflers built during the completion of his dissertation that consisted of an empty cylindrical expansion chamber with no internals (6). The researcher decided to select one of these mufflers as the base configuration for the completion of this thesis. The dimensions of the expansion chamber are shown in Figure 3.7. The overall dimensions of the muffler are relatively large compared to most mufflers in application. That said, as parametric studies were being conducted, the muffler became significantly more complicated and there was very large attenuation in the exhaust system. This led to some initial difficulties in achieving accurate experimental data for the more complicated mufflers. All of the correlated experimental models developed will be discussed in the next chapter. 


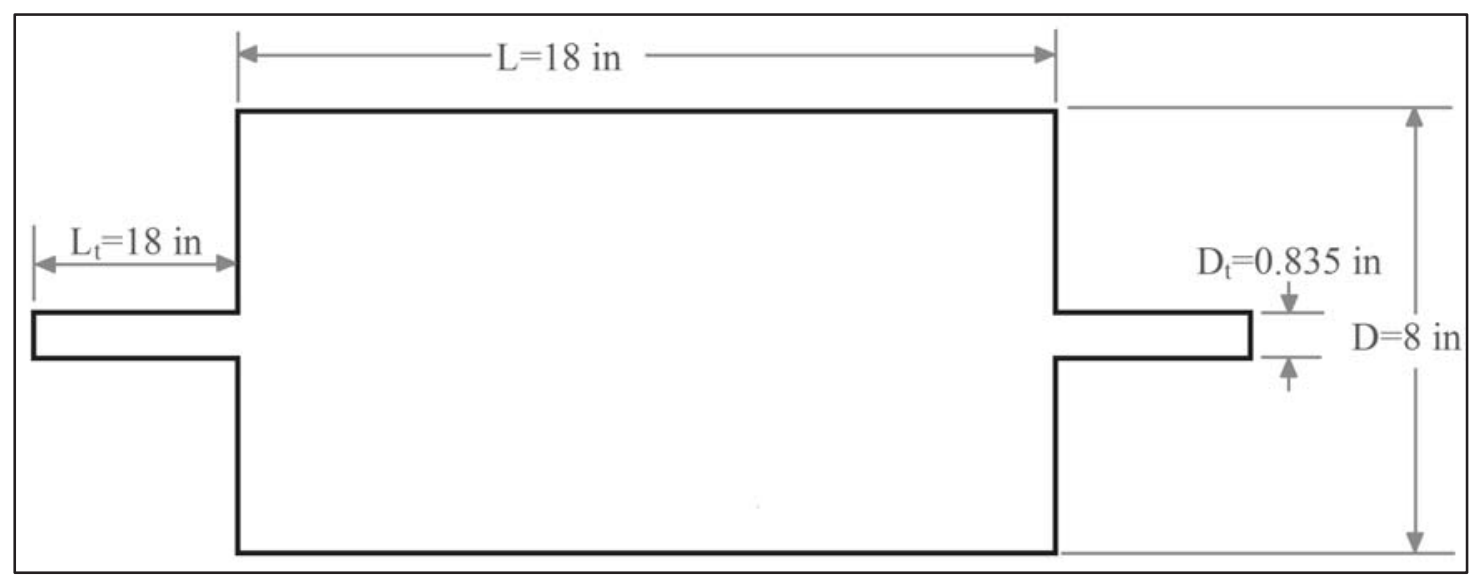

Figure 3.7: The overall dimensions of the baseline muffler used while completing this thesis.

The elements that were tested for the simulation and the experimental study were selected to determine the effects of extended tubes (quarter wave resonators), perforated tubing, internal baffles, overall chamber dimensions, absorptive material, and mass flow rate through the system. Temperature effects were only examined in the simulation models. All of the internal components needed to be purchased and fabricated for the completion of this thesis. The extended tubes were cut on the lathe to allow for installation of different perforated lengths. The perforated tubing used had a ID of 0.835 ", thickness of 3/64", hole diameter of $1 / 8$ ", hole spacing of 1/4", and a hex hole pattern. The tube was weldless which has been known to produce the best performance. Using Mechel's formula shown in Equation 2-32, the impedance properties of the perforated tubing could be calculated. The ends of the perforated tubing were capped shut so the sound waves were forced through the perforations. A picture of the perforated tubing and extended tubes used is shown in Figure 3.8. 


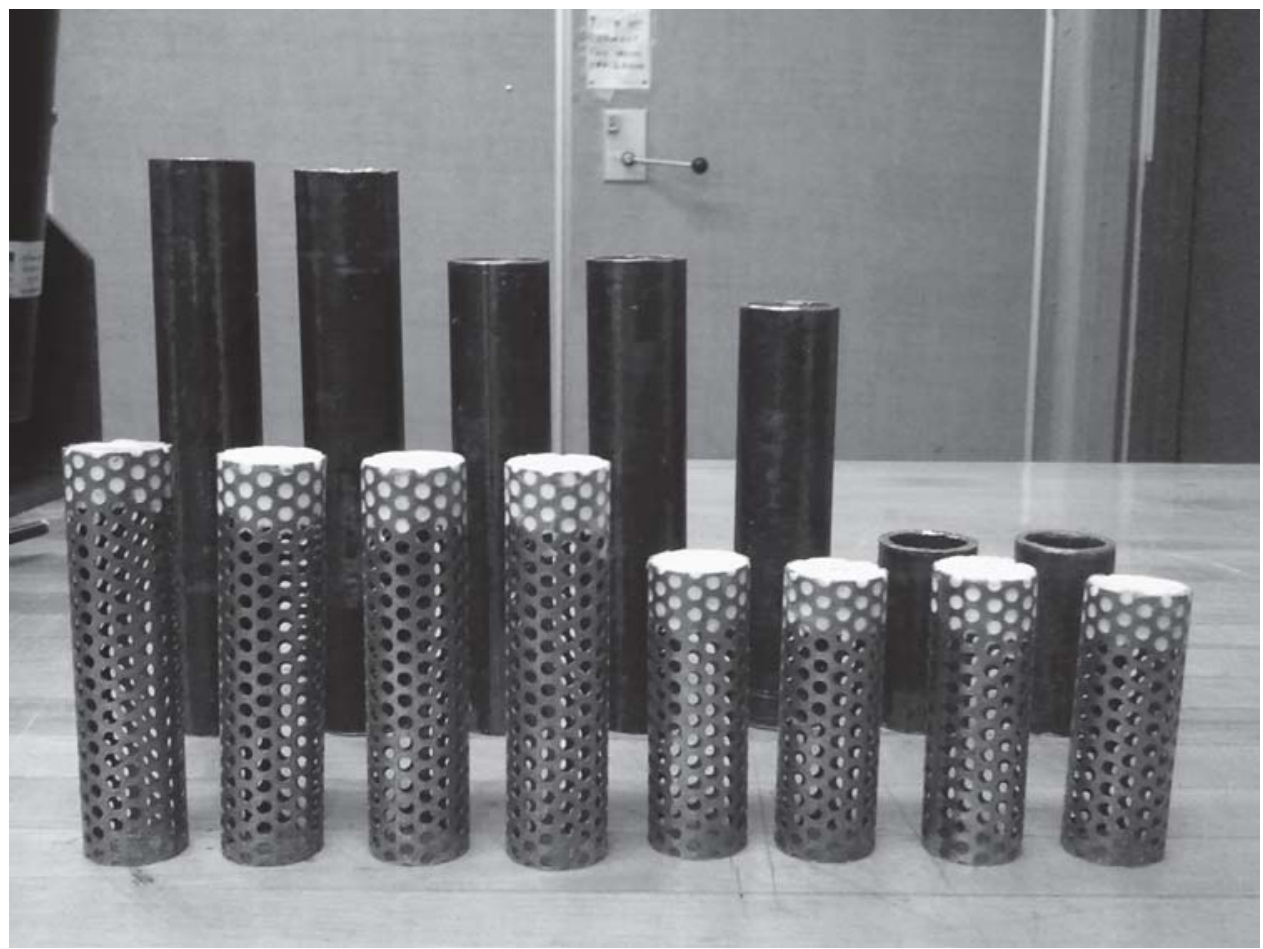

Figure 3.8: A picture showing the extended tubes and perforated tubing that was used during the completion of this thesis.

The internal baffle that was used was made from 1/2" plywood and the small crevices in the wood were filled with plaster to produce the most optimum results. A difficult situation that arose was because the simulation models were created before the experimental models were developed, which had the baffle modeled as infinitely thin. Although the dimensions of the internal chambers were developed to match the simulation, the thickness of the baffle proved to significantly affect the performance of the TL. Because of this, the researcher felt it necessary to create all new models which incorporated the thickness of the plywood. The findings will be presented in Chapter 5. A picture with the internal baffle installed with extended and perforated tubing is shown in Figure 3.9. 


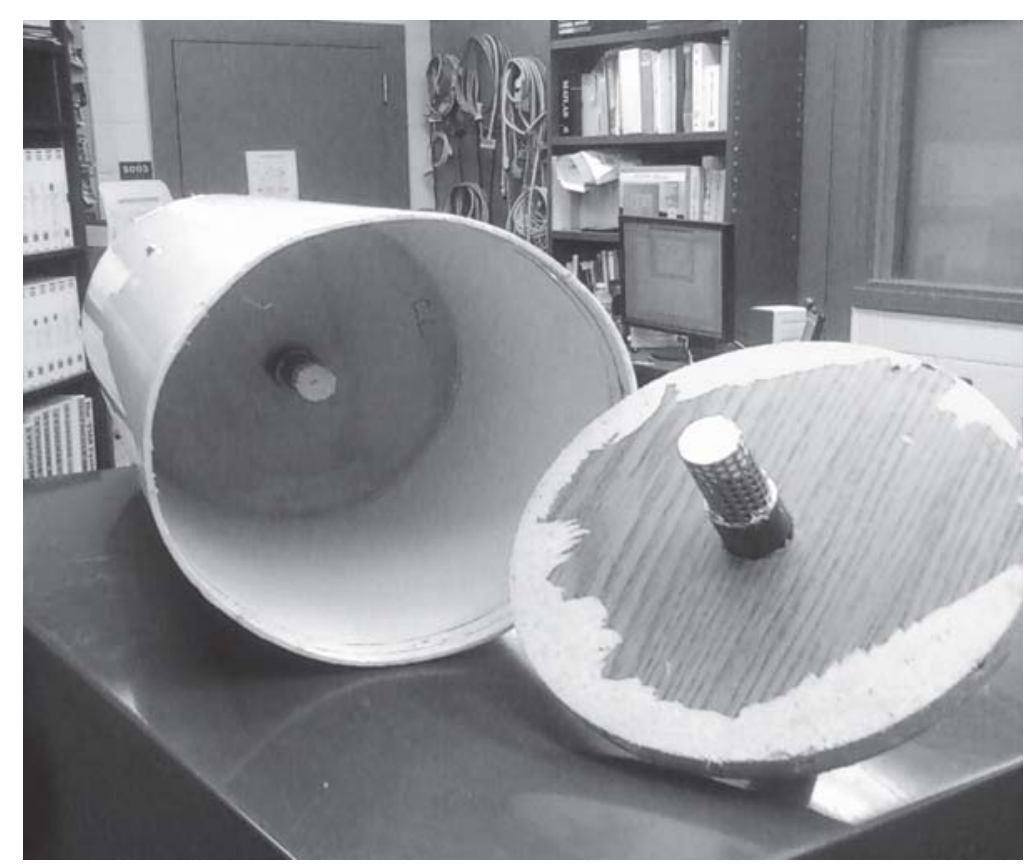

Figure 3.9: A picture showing the internal baffle, extended tubes, and perforated tubing used for one of the correlated experimental models.

The absorptive material used was BGF Industries, Inc.'s SilcoSoft. The material is a woven fiber that had a thickness of roughly $1 / 4$ " and a density of about $8 \frac{l b}{f t^{3}}$. As mentioned previously in Section 2.4.5, the material properties of nearly all glass fiber materials have roughly the same material properties. The main property, resistivity $\sigma$, can vary fairly significantly with minimum differences and will be verified in Chapter 5. Because of the similarities between common glass fiber materials, the material properties of the glass woven fiber HKO were used for this thesis. The material properties of HKO were measured on another project and has a resistivity given in Equation 3-8, where $t$ represents the thickness of the material. The porosity of the material was $92 \%$ and the structural factor was taken as 1, a common value for these types of materials. A picture of this material installed in the muffler is shown in Figure 3.10.

$$
\sigma\left[\frac{N s}{m^{4}}\right]=\frac{2177.5}{t[m]}
$$




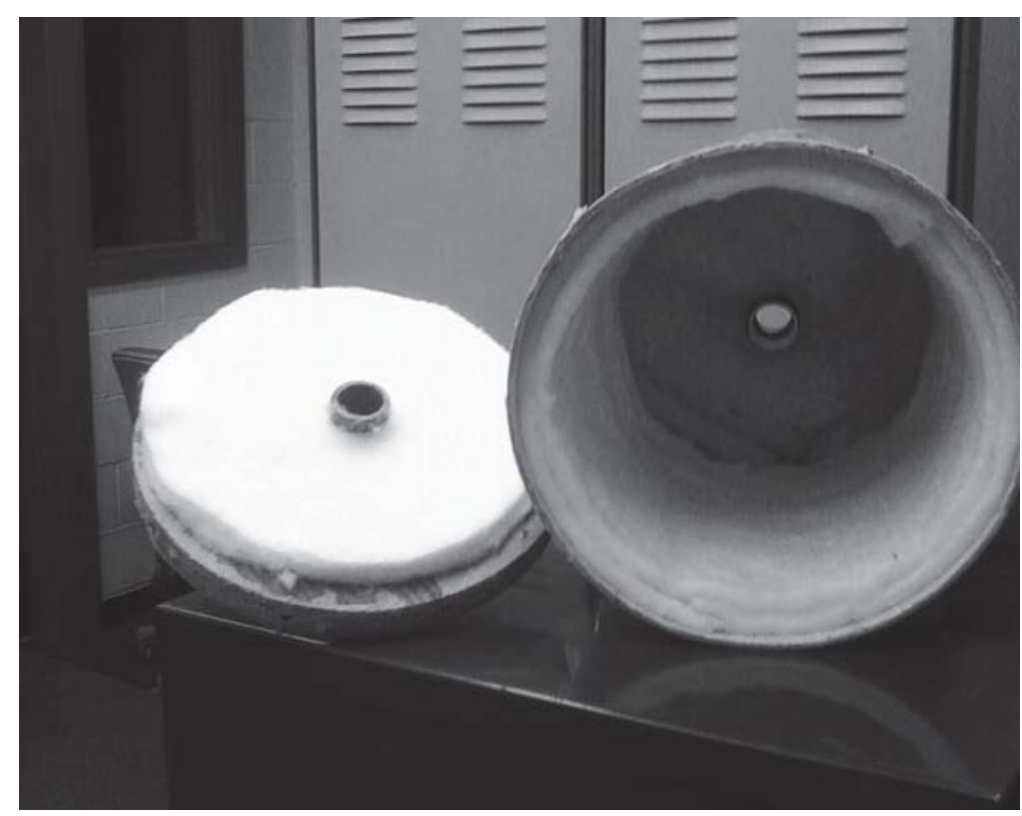

Figure 3.10: Two layers of absorptive material installed in the muffler. 


\section{Chapter 4. Simulation Method}

The main focus during the research for this thesis was examining and learning different simulation tools for predicting muffler performance. The author had no previous experience in Finite Element Analysis (FEA) nor with the simulation package used (LMS's Virtual.Lab). This proved to be a very time consuming and valuable process. Due to the author's previous knowledge (or lack thereof), it was not appropriate for the author to perform muffler analysis on all of the available methods. As a result, it was first important to understand the benefits and drawbacks of each method and then determine which ones would be practiced, accordingly.

\subsection{Comparison of different muffler evaluation methods}

When determining what type of method to use for calculating muffler performance, it is critical to address what type of effects are to be examined as well as the assumptions to be made. There typically is one method that may be more beneficial to use than another given the conditions stated previously. However, in order to best model the entire system, including the source, it is most beneficial to use a coupled model that implements both CFD and FEM/BEM techniques. The different techniques will be presented in this section and their best application with respect to modeling an exhaust system will be discussed.

\subsubsection{1-D linear and non-linear techniques}

One of the main uses for 1-D solvers is for performing non-linear (time domain) calculations when modeling the thermodynamic cycle of the engine. This 
determines the unsteady gas flow in the exhaust pipe. As stated in Section 2.7, varying loads can be applied to the model to then determine the source impedance and source pressure by formulating an over determined set of equations. This information is a necessity if Insertion Loss or SPL measurements are to be predicted.

The Transmission Loss of mufflers can be calculated using onedimensional software codes such as GT-Power or Wave. The 1-D packages will solve a linear (frequency domain) solution for the transfer matrix method for common muffler elements as presented in Section 2.2 (21). This type of method typically cannot model complicated muffler components and only plane waves can exist in the model (26). The effects due to three-dimensional waves cannot be predicted which usually occurs in the middle to high end of the frequency spectrum. In commercial mufflers, where there is a fairly large expansion in the muffler, 3-D wave propagation occurs low in the frequency spectrum which poses as a problem for 1-D solvers.

As a result, 1-D muffler TL testing should only be used as a precursor to a different method and has been proven to only provide good correlation at fairly low frequencies. However, the time savings both setting up and performing 1-D simulations is substantial compared to the other methods. When used in the right situation, this tool can be very effective.

\subsubsection{3-D CFD}

The 3-D CFD codes commonly used for muffler analysis are programs such as Fluent and STAR-CD. These programs use the RNG k- $\varepsilon$ turbulence model that describe the fluid dynamic properties of a compressible fluid medium seen in 
turbulent flow (16). This type of non-linear simulation is extremely useful in determining the flow and temperature patterns inside of the exhaust system. This is completed by applying the engine operating conditions as an initial condition to the model (29). The flow and temperature profiles can be used as an input into a linear solver, such as the FEM, which can be used to calculate the TL with these additional effects.

The downside is that the CFD techniques are the most time consuming and computationally costly. Although, with the advancement in computer technology, their popularity is growing exponentially. Due to the time requirement to learn CFD techniques, it was deemed unfeasible for the application of this method in this thesis. These techniques are considered the most effective method in determining the effects of temperature gradients and flow generated noise in an exhaust system however. It would be recommended that a continuation of this thesis would incorporate 3-D CFD methods.

\subsubsection{BEM and FEM method}

Both the Boundary Element Method and the Finite Element Method are considered the linear (frequency domain) approach which is based on the fundamental 3-D wave equation as demonstrated in Equation 4-1. From this equation, the state variables (pressure and particle velocity), can be determined for every nodal point in the model at each frequency spectral line.

$$
\nabla^{2} p+k^{2} p=0
$$

Equation 4-1

The BEM is composed of two-dimensional shell elements which make up all of the surfaces of the muffler. On the other hand, FEM is a three-dimensional 
mesh which needs to be created for the entire exhaust system cavity. Because of this, the FEM mesh will contain well over an order of magnitude more nodes and elements than its counterpart, the BEM (12). If the same computational solver is used for each method then the BEM can serve as a major computational advantage. The downside is that the state variables are only known on the surfaces of the muffler for the BEM whereas using the FEM the state variables are known at every nodal point inside the muffler.

For the BEM in order to determine the state variables inside the muffler and exhaust tubes, field points need to be created. The state variables are solved at these locations as a post-processing step after the BEM simulation and are determined by an integration technique from nearby nodes (6). Careful attention needs to be made when selecting locations for the field points because if the location is too close to a nearby node (less than one element length) than inaccurate results may be produced. Also for the BEM, plane waves must exist at the location of the field points for their state variables to be accurately predicted. In order to force plane wave propagation in the exhaust tubes, there should be spacing equivalent to approximately six tube diameters before and after the field point. For the TL calculation, the inlet tubes can be extended to account for this and the overall TL will not be affected in the process. However, the overall impedance of the muffler will be effected. The inlet and outlet tube lengths are very important for correct phasing on the impedance. This, in-turn, will affect the IL or SPL prediction.

Using the FEM approach, the location of the measurement point inside of the inlet and outlet tube is not effected by the placement as seen using the BEM method. The initial investigations of the TL calculations for the research conducted for this thesis used the harmonic BEM using LMS's Virtual.Lab Acoustics module. The researcher saw very significant differences for the TL of a 
simple expansion chamber dependent upon the location of the field point. This was not the case when using the FEM model. The BEM technique also suffers being unable to accurately model complicated muffler designs that include absorption material and perforated tubing. The FEM is far superior in this category. The BEM technique cannot be used to incorporate mean flow or temperature gradients as well.

Over the past few years, the Sysnoise solver for LMS's Virtual.Lab Acoustics has seen drastic improvements for computational time of FEM Acoustic models which implements Krylov solving methods. Because of these advances, the benefits of using BEM over FEM techniques for solving muffler performance properties are constantly decreasing. The actual setup time of the models for both the BEM and FEM are fairly similar, however, FEM typically would take slightly longer to mesh. Since there is higher accuracy in TL because the state variables are calculated for interior nodes, the FEM was implemented for all of the parametric studies done in this thesis.

These two types of methods are most effective in determining the TL of mufflers when steady state conditions are considered. Their downfall is whenever the source is non-periodic. This situation may exist when examining only one complete cycle of engine combustion or when determining the temperature pattern in a muffler as a function of time. In order to incorporate these non-periodic effects, CFD methods need to be used and the results incorporated into the model.

\subsection{Setting up an acoustic FEM model in VL}

This section will go through the process of designing a muffler model and performing a TL calculation using FEM techniques in LMS's Virtual.Lab. 
Additional steps will be given for implementation of source data and determining SPL in the free field.

\subsubsection{Solid model generation}

The first step, as with any FEM simulation, is to start with the design of the model. In this thesis, the researcher learned the solid modeling package CATIA which was used to create the muffler models. When designing the mesh, only the skins of the muffler are needed. Accordingly, the muffler can either be modeled using sheets or the skins need to be extracted from the solid geometry. Each component should be created using a separate body in the part to allow for easy distinction between the components when generating the mesh. A picture showing one of the wireframe muffler models used in the research is shown in Figure 4.1. After the model is created it needs to be output to a readable format for the mesh generating software. The model can be saved as either a step file (.stp) or Initial Graphics Exchange Specification file (.iges), however, a step file is recommended.

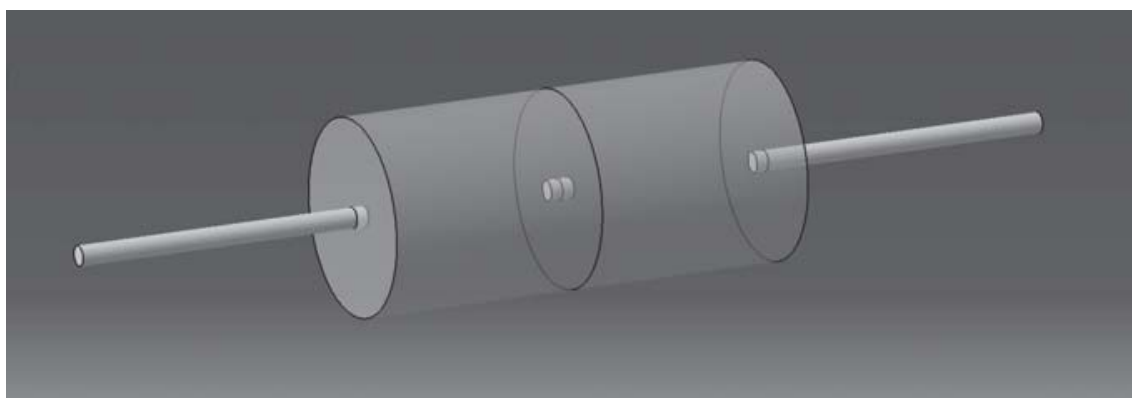

Figure 4.1: The CAD model for one of the mufflers used in this thesis. 


\subsubsection{Mesh creation}

The meshing software used in this thesis was Altair's HyperMesh 11.0 because of the sophisticated control over the mesh generated. As the researcher had no previous knowledge of the software or meshing in general, this proved to be one of the major learning processes. Each of the muffler components created inside of the muffler should have their own mesh component. This helps to decrease the model setup time in VL significantly. Then each surface of every component needs to be meshed using 2-D elements. The typical criterion for acoustic meshes is that the element size should be small enough to allow at least six elements to fit in the wavelength of the max frequency of interest (18). For the models used in this thesis, the maximum element size was set to 1/4". This would result in the max achievable frequency of $9000 \mathrm{~Hz}$; well over the max frequency of interest $(3000 \mathrm{~Hz})$. One of the meshes created for this thesis is shown in Figure 4.2. Another important factor is mesh quality which can negatively affect the maximum measureable frequency. The mesh quality can be calculated directly in HyperMesh and the low scoring elements can be singled out and corrected. Usually, a poor aspect ratio or substantial differences of internal element angles result in a degraded mesh quality.

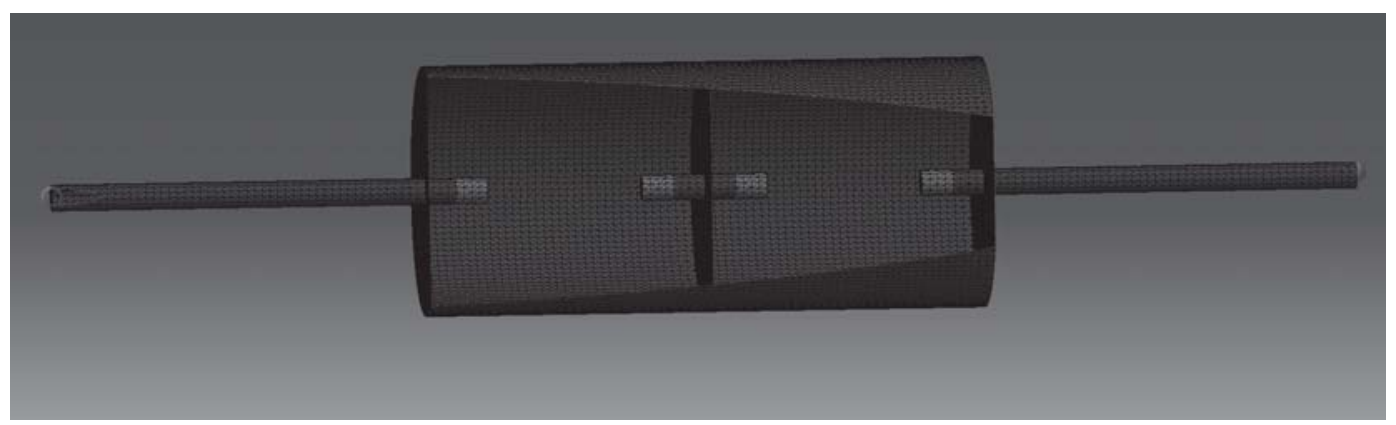

Figure 4.2: A mesh created for a dual expansion chamber with extended tubes and perforations using $1 / 4$ " elements. 
It is advisable that surfaces inside of the muffler, such as extended tubes, share coincident (although uncommon) nodes between the inside surface elements and the outside surface elements. After creating the 2-D elements, all of the internal components inside of the muffler should be completely closed. At interfaces where two components meet and the pressure waves are allowed to flow freely from one component to the next, the components should share common and coincident nodes. For this thesis, this was done by making common nodes in HyperMesh and then merging the nodes in LMS's nodes and elements module.

After creating the 2-D mesh, a 3-D tetramesh (which is a common element type in acoustical meshes) can be created for each component. The 2-D elements should either be deleted or placed in a different component after this step and should not be exported into Virtual.Lab with the tetramesh. The newly created mesh can be exported into a general readable format such as a bulk data file (.bdf) which can be imported into Virtual.Lab. If the muffler is fairly sophisticated, the meshing of the muffler is the most time consuming process which can take over a day for the most complicated mufflers. Another shortcoming is that for every design iteration, the effected components need to be remeshed which can take nearly as much time to complete.

\subsubsection{Importing acoustic mesh and assigning properties}

After the mesh is imported into Virtual.Lab's Acoustic Harmonic FEM module, it needs to be assigned as an acoustical mesh. All the necessary nodes and elements at interfaces between components need to be merged together which can be done in the Nodes and Elements module. The next step is to create an acoustic mesh preprocessing step in FEM. The preprocessing prepares the mesh for an acoustic analysis and creates the acoustic envelope of the muffler. The acoustic 
envelope of the muffler represents the boundary surfaces of the muffler components where boundary conditions are applied (24).

At this point, groups should be created which may represent elements with different material properties, surfaces were perforated holes will be placed, inlet and outlet locations, etc. It should be noted that the inlet and outlet groups should be created from the acoustic envelope as they will be used to place boundary conditions. The material properties can be created once the groups are generated. In the parametric studies completed, a material property for air was created which initially was set to the default values for a speed of sound of $340 \mathrm{~m} / \mathrm{s}$ and density of $1.225 \mathrm{~kg} / \mathrm{m}^{3}$. After conducting the experimental tests, these values were fairly off as the temperature of the room was higher than expected. Using Equation 2-38 and Equation 2-40, the new values seen during experimental testing were $348 \mathrm{~m} /$ $s$ for the speed of sound and $1.1785 \mathrm{~kg} / \mathrm{m}^{3}$ for the density of the acoustic fluid. As a result, all of the simulation work done needed to be recomputed. If the medium had different properties in various areas of the muffler, as in the case if experimental temperature data was collected for different locations, then multiple material properties need to be created.

The next step is to assign the material properties to the appropriate section. This would include any absorptive material used. In this thesis, the baseline values for the absorptive materials used are presented in Section 3.4 and the parameters for porosity, resistivity, and structural factor were adjusted to see their effects. As the holes in the perforated tubing are far too difficult to mesh and lead to inaccurate results, a transfer admittance property can be assigned to areas where perforations are present. It should be noted that two surfaces need to be selected for this and must have common node locations. The baseline values for the perforated tube properties were presented in Section 3.4 and parameters for hole 
size, spacing, and porosity were modified to see the effects. The calculation for the equivalent impedance is shown in Equation 2-33 and Equation 2-34.

\subsubsection{Input and boundary conditions}

In simulation it is required that the termination at the outlet is to be fully anechoic. This can be easily accomplished by applying an absorptive panel property for the group assigned to the outlet. The absorptive panel should have an equivalent impedance of $\rho c$ (typically $416.5 \mathrm{~kg} / \mathrm{m}^{3} \mathrm{~s}$ ) which would mean that there would be no reflective waves in the tailpipe. The input boundary condition should have a unit velocity of $1 \mathrm{~m} / \mathrm{s}$ applied to the inlet face of the muffler.

If the steady state effects of mean flow on sound propagation are to be modeled, a mean flow boundary condition set needs to be created (30). The mean flow boundary condition is set on the inlet, which for this thesis ranged from 0.05 Ma to $0.35 \mathrm{Ma}$. On the outlet, a zero flow potential should be assigned to simulate the flow into the atmosphere. The next step is to solve for the potential flow solution and then couple the results to the Acoustic Response Analysis case. This method does not work for perforations which exist in most commercial mufflers. Also the solution time is substantially greater. The models simulated took nearly ten times as long to solve as compared to not having the mean flow effects.

\subsubsection{Job creation and post processing}

After the model is all set up, an Acoustic Response Analysis case can be added to the project. In the dialog box you can upload the preexisting boundary conditions and the mean flow solution if applicable. This is the frequency domain 
approach; this allows the user to designate what frequencies are to be solved. For this thesis, originally $5 \mathrm{~Hz}$ spacing was initially implemented. Eventually the researcher deemed it unnecessary and switched to $10 \mathrm{~Hz}$ spacing to decrease the computation time. The starting and ending frequencies were 1 and $3000 \mathrm{~Hz}$, respectively. If field points were added than it becomes necessary to save the results of the field points as vectors. The solutions at each frequency do not need to be solved in any particular order, allowing you to have the ability to perform parallel processing. In which case, a different frequency calculation is conducted on various computer processors, simultaneously. This can greatly speed up computational time, especially for high end computers that have eight processors.

The results from the processing will be vector solutions for the state variables at every node for each spectral line. Since TL is calculated for specific elements as a function of frequency, a vector to function conversion case needs to be created. The initial step is to create an IO set, this designates which nodes will be used as the inlet and outlet. Following, the vector to function conversion can be performed for the specific nodes of interest.

At this point, the TL of the exhaust system may be calculated. The equation to be used to calculate TL is shown in Equation 2-21. In the equation, the mass velocity is the velocity assigned as the boundary condition to the inlet which is equal to $1 \mathrm{~m} / \mathrm{s}$. The acoustic pressure wave is complex; therefore the measured pressure signal can be multiplied by its complex conjugate resulting in the absolute squared term for the signal. 


\subsubsection{SPL prediction using FEM}

In order to determine the sound pressure level in the free field produced by a coupled engine-exhaust system model, the source properties need to be determined in the steady-state frequency domain. The input velocity is a function of both source and muffler impedance. Therefore, the first step is to determine the 4-pole parameters of the muffler for the actual operating condition. This is completed the same way as done experimentally, in Section 3.1.2, using the twosource location method. In the calculation of the 4-pole parameters, it is crucial that an accurate temperature profile is applied to the model. Slight differences in the speed of sound can lead to major differences in SPL predictions because the output spectrum is relatively narrow band. The impedance of the muffler can now be calculated using Equation 2-47.

Using the one-port model as described in Section 2.7, the source impedance and source pressure can be calculated. Virtual.Lab can only have impedances and velocities as boundary conditions; consequently, the source pressure needs to be converted to a velocity. This is done using Equation 2-51, where the muffler impedance calculated by the four pole parameters is the impedance downstream of the muffler. The calculation incorporates muffler impedance seen upstream so the muffler impedance needs to have a 180 degree phase shift applied to it.

Once the boundary conditions are known, they can be applied to the model. The velocity boundary condition is applied the same way as before, however, this time the velocity would be frequency dependent. The source impedance would be applied as an absorptive panel property with a frequency dependent impedance boundary type. In order to simulate the free field, a sphere needs to be created and attached to the end of the outlet. The sphere than should be assigned as an Automatically Matched Layer property (AML). This represents the sound radiating from the tailpipe to the atmosphere. The AML property is a recent 
advancement which allows you to place a field point anywhere in free space and no additional node or elements are needed in between the AML layer and the point. Reflective surfaces can also be added to the model to represent the ground. If the overall sound power is wanted, a spherical field point mesh can be created which surrounds the complete exhaust system.

\subsection{Muffler Testing in VL}

As mentioned previously, the biggest portion of this thesis was conducting an FEM parametric study of the effects of various elements on muffler performance. The baseline configuration, which all of the parametric configurations are based off of, is shown in Figure 3.7. Table 4.1 shows the different configurations for each parametric study. The dimensions that are underlined were models that were developed experimentally as well and served for correlation purposes.

Table 4.1

A description of the parametric studies completed throughout the duration of this thesis.

\begin{tabular}{|c|c|c|}
\hline $\begin{array}{c}\text { CASE } \\
\text { Number }\end{array}$ & Description & Dimensions \\
\hline $\mathbf{1}$ & Change expansion tube diameters & $6^{\prime \prime}, \underline{\boldsymbol{8}^{\prime \prime}, \text { and } 10^{\prime \prime}}$ \\
\hline $\mathbf{2}$ & Change length of expansion tube & $12^{\prime \prime}, 18^{\prime \prime}$, and 24" \\
\hline $\mathbf{3}$ & Change length of inlet/outlet tubing & $2 ", 4 ", 6 ", 12^{\prime \prime}$ \\
\hline $\mathbf{4}$ & Change the diameter of the inlet/outlet & $.835^{\prime \prime}, 1 ", 1.5^{\prime \prime}$ \\
\hline
\end{tabular}


Table 4.1, cont'd.

A description of the parametric studies completed throughout the duration of this thesis.

\begin{tabular}{|c|c|c|}
\hline $\begin{array}{c}\text { CASE } \\
\text { Number }\end{array}$ & Description & Dimensions \\
\hline 5 & $\begin{array}{l}\text { Addition of extended tubes to single } \\
\text { expansion chamber }\end{array}$ & $\underline{\text { none, }} 0.5^{\prime \prime}, \underline{1^{\prime \prime}}, \underline{2^{\prime \prime}}$ \\
\hline 5.1 & $\begin{array}{c}\text { Addition of perforated tubing with 1" } \\
\text { extended tubes }\end{array}$ & none, $\underline{1 "}, 2^{\prime \prime}$ \\
\hline 5.1.1 & $\begin{array}{l}\text { Modifying perforations properties of 1" } \\
\text { perforated tubing and } 1 \text { " extended tubes }\end{array}$ & See Notes \\
\hline 6 & $\begin{array}{c}\text { Changing the number of expansion } \\
\text { chambers }\end{array}$ & $\underline{1}, \underline{2}, 3$ \\
\hline 7 & $\begin{array}{l}\text { Addition of extended tubes to dual } \\
\text { expansion chamber }\end{array}$ & $\underline{\text { none }}, 0.5^{\prime \prime}, \underline{1^{\prime \prime}}, 2^{\prime \prime}$ \\
\hline 7.1 & $\begin{array}{c}\text { Addition of perforated tubing with 1" } \\
\text { extended tubes }\end{array}$ & none, $\underline{1 "}, 2^{\prime \prime}$ \\
\hline 7.1.1 & $\begin{array}{l}\text { Modifying perforations properties of } 1 " \\
\text { perforated tubing and } 1 " \text { extended tubes }\end{array}$ & See Notes \\
\hline 8 & $\begin{array}{l}\text { Addition of absorptive material to } 1 " \\
\text { extended tube dual camber muffler }\end{array}$ & $\underline{\text { none, }}, \underline{.25^{\prime \prime}}, \underline{.5 "}, 1 "$ \\
\hline 8.1 & $\begin{array}{l}\text { Changing absorptive material properties } \\
\text { for the } 1 / 2 \text { " configuration }\end{array}$ & See Notes \\
\hline 9 & $\begin{array}{l}\text { Adding mean flow through single empty } \\
\text { expansion chamber }\end{array}$ & 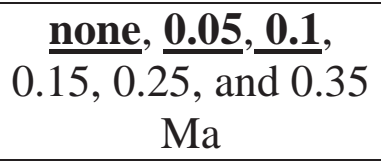 \\
\hline 10 & $\begin{array}{l}\text { Adding mean flow through dual expansion } \\
\text { chamber with } 1 \text { " extended tubes }\end{array}$ & $\begin{array}{l}\text { none, } 0.05,0.15 \\
0.25, \text { and } 0.35 \mathrm{Ma}\end{array}$ \\
\hline 11 & $\begin{array}{l}\text { Effects of temperature on dual expansion } \\
\text { chamber with } 1 " \text { extended tubes }\end{array}$ & $\begin{array}{l}20 \mathrm{C}, 100 \mathrm{C}, 200 \mathrm{C}, \\
400 \mathrm{C}\end{array}$ \\
\hline
\end{tabular}


The perforated tubing properties used to calculate the transfer admittance properties of the tubes are described in Table 4.2. The equivalent impedance of the perforated tubing was calculated using the MatLab script created, in Appendix B. The underlined item represents the perforated tubing used during the experimental analysis.

When performing the experimental TL testing with the incorporated mean flow, the experimentalist felt it was necessary to use the 6" diameter control muffler rather than the 8" diameter muffler used in the parametric study. This was because the experimentalist knew that a good measurement was going to be very difficult with the flow noise generated in addition to the attenuation of the muffler. This provided the experimentalist with an attenuation of few $\mathrm{dB}$ less which could help produce more accurate results. All of the other dimensions of the new control muffler were the same.

The test with mean flow consisted of two configurations. The first being the empty expansion chamber and the second where 1" perforated tubing was added to the inlet and outlet of the muffler. The TL was measured for $0,0.05$, and $0.10 \mathrm{Ma}$ flow. The 0.10 Ma flow was measured to be a flow velocity of roughly $35 \mathrm{~m} / \mathrm{s}$. The testing was conducted using the Stepped-Sine approach as mentioned previously.

Table 4.2

Perforated tubing properties for specimens used in study.

\begin{tabular}{|l|c|c|c|c|c|}
\hline $\begin{array}{c}\text { Perforated } \\
\text { tubing } \\
\text { configuration }\end{array}$ & $\begin{array}{c}\text { Hole } \\
\text { Diameter } \\
(\mathbf{m m})\end{array}$ & $\begin{array}{c}\text { Hole } \\
\text { spacing } \\
(\mathbf{m m})\end{array}$ & $\begin{array}{c}\text { Hole } \\
\text { pattern }\end{array}$ & $\begin{array}{c}\text { Tube } \\
\text { thickness } \\
(\mathbf{m m})\end{array}$ & $\begin{array}{c}\text { Porosity } \\
(\boldsymbol{\%})\end{array}$ \\
\hline Baseline & $\underline{3.175}$ & $\underline{6.35}$ & $\underline{\text { hex }}$ & $\underline{1.2}$ & $\underline{13.60 \%}$ \\
\hline Property \#1 & 2 & 9 & hex & 1.2 & $10.75 \%$ \\
\hline Property \#2 & 8 & 9 & hex & 1.2 & $43.00 \%$ \\
\hline Property \#3 & 2 & 4.5 & hex & 1.2 & $43.00 \%$ \\
\hline
\end{tabular}


The author had to rely on experimental methods in determining some of the effects from flow noise since CFD was not practiced. The flow noise inside of the tubing was measured at the reference location downstream of the muffler for three different muffler configurations. The first two configurations were the same two used during the mass flow TL testing, stated previously. The third configuration was the 8 " diameter muffler with dual expansion 1" extended tubes and 1/2" of absorptive material. This muffler was selected because it was already built from the parametric study and shows the effects of flow noise in a more complicated muffler design. The testing was done at various flow speeds and the source was used as both a vacuum and a blower. Air flow run-ups were also performed to clearly show the relationship between air velocity and flow noise. 


\section{Chapter 5. Results}

\subsection{Parametric studies}

This chapter includes all of the simulation work done for the parametric studies. Again, the baseline control muffler is shown in Figure 3.7. Properties of the elements used were presented in Chapter 3 and Chapter 4. All of the FEM models needed to be rerun because of two factors. First was that the acoustic fluid properties did not initially match the fluid properties during experimentation. Secondly, the center baffle in the dual chamber design was initially modeled as infinitely thin which lead to poor correlation as will be shown in Section 5.2. Because of these two factors, the revision number, $r 0$, represents the initial simulations and the revision number, $r 1$, contains the correct fluid properties and correct thickness for the baffle. For the single expansion chamber design, only one simulation will be shown and both simulations will be presented for the dual expansion chamber studies.

\subsubsection{Case 1 - Change expansion tube diameter}

In this study, the overall expansion tube diameter was changed to diameters of 6", 8", and 10". The results using the plane wave method were identical to the FEM method up to the point where higher order modes start to propagate. The TL results for the three different models are in Figure 5.1. As you can see in the figure, the amplitude of the peak for the transmission loss of a simple expansion chamber is governed by the overall diameter of expansion into the chamber. The downside is that due to the larger diameter the 3-D wave propagation occurs 


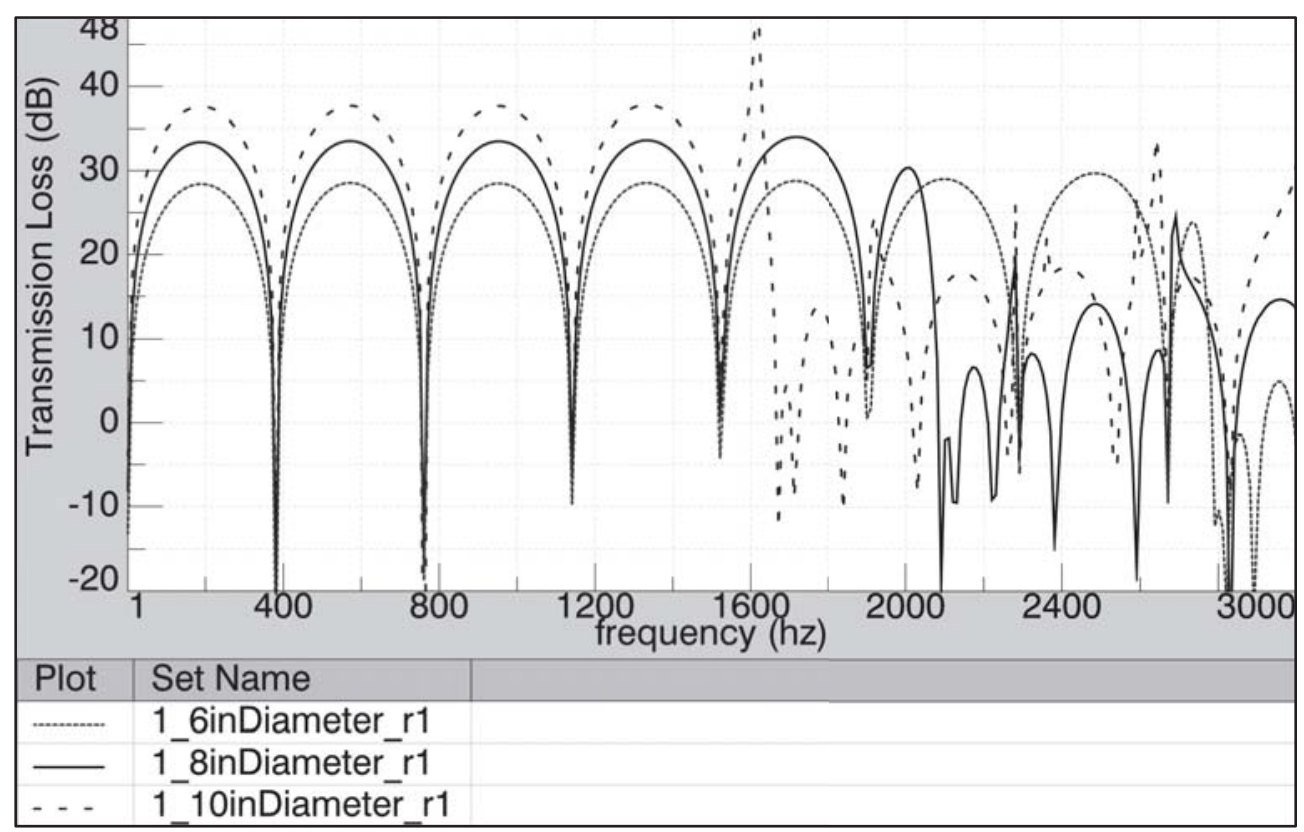

Figure 5.1: Case 1 parametric study - the diameter of the expansion tube was modified for the single expansion chamber.

earlier in the frequency spectrum. Because the inlet and outlet tube are placed in the center of the baffle, the higher order diametrical effects are not seen in the TL. As a result, higher order effects are first seen at the first axisymmetric mode. This occurs at a frequency of $2800 \mathrm{~Hz}, 2100 \mathrm{~Hz}$, and $1650 \mathrm{~Hz}$ for the 6",8", and 10" diameter muffler, respectively. Typically, the 3-D wave propagation has a detrimental effect on TL, as demonstrated in the figure.

\subsubsection{Case 2 - Change the overall length of the empty expansion chamber}

The next case is to determine how the overall length of the empty expansion chamber affects TL. The length of the expansion chamber was varied to lengths of 12,18, and 24 inches and the TL can be seen in Figure 5.2. As shown in the figure, the length of the expansion chamber has no effect on the maximum amplitude of the domes as well as the location of the cut off frequency. These 
effects are accounted for by the overall diameter of the expansion tube. What the length does affect is the overall width of the domes. This is determined from Equation 5-1 where $L$ represents the length of the tube (2). The findings on the previous two investigations can greatly assist a designer in finalizing a muffler design. If the TL needs to be shifted to the left slightly (if a major firing order doesn't line up near a peak in TL), it would be more advantageous to increase the length of an expansion chamber. On the other hand, if the major firing order does already line up on a peak it would be more appropriate to increase the expansion diameter for further attenuation. These conclusions have been proven to hold true for much more complicated mufflers.

$$
f=\frac{2 c}{L}
$$

Equation 5-1

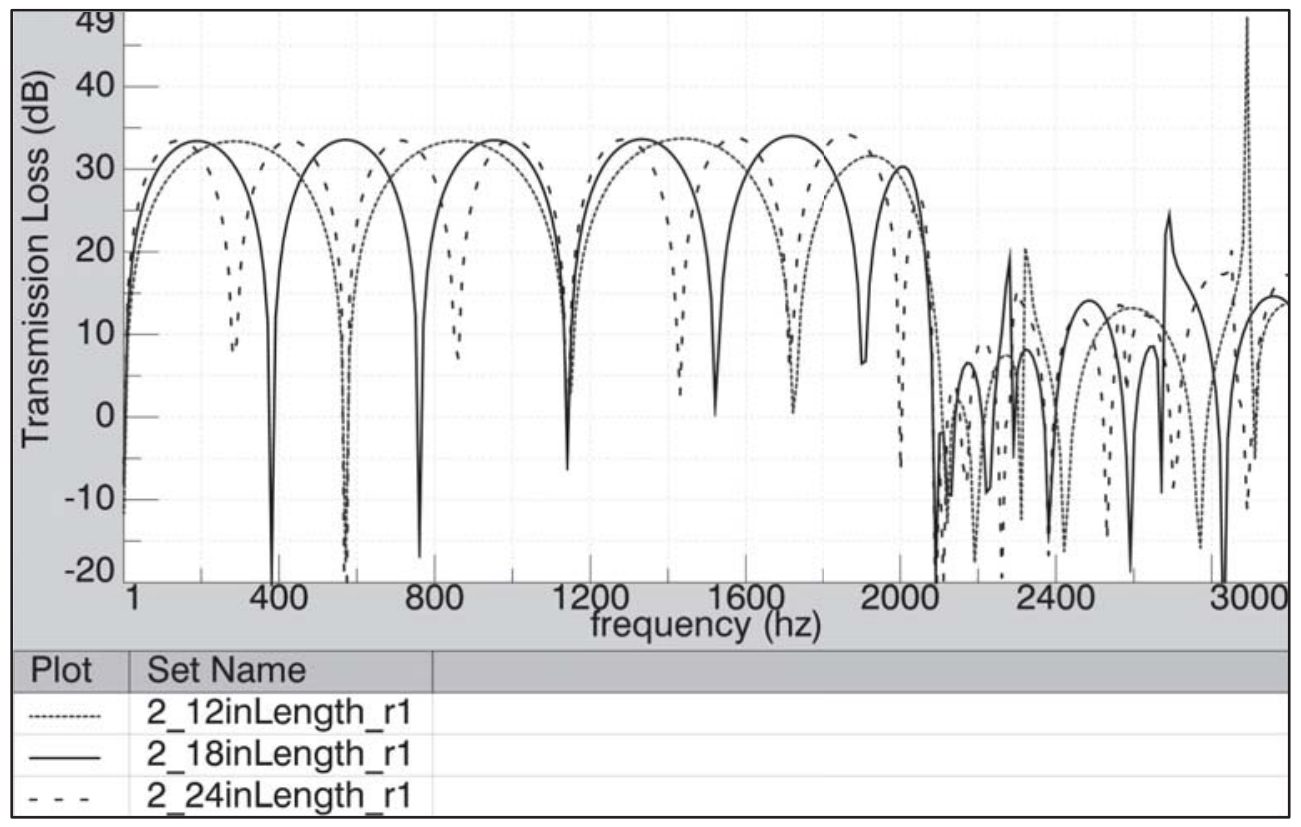

Figure 5.2: Case 2 parametric study -The length of the expansion chamber is varied for the single expansion chamber. 


\subsubsection{Case 3 - Change the length of the inlet and outlet tube with single expansion chamber}

The length of the tubes connecting the muffler was changed to lengths of 2 , 4,6 , and 12 inches for this study. This study was investigated to confirm that the location of the field point in the muffler inlet and outlet does not significantly affect the TL using the FEM, whereas, in the BEM this was not the case. The findings confirm the reasoning stated for using the FEM.

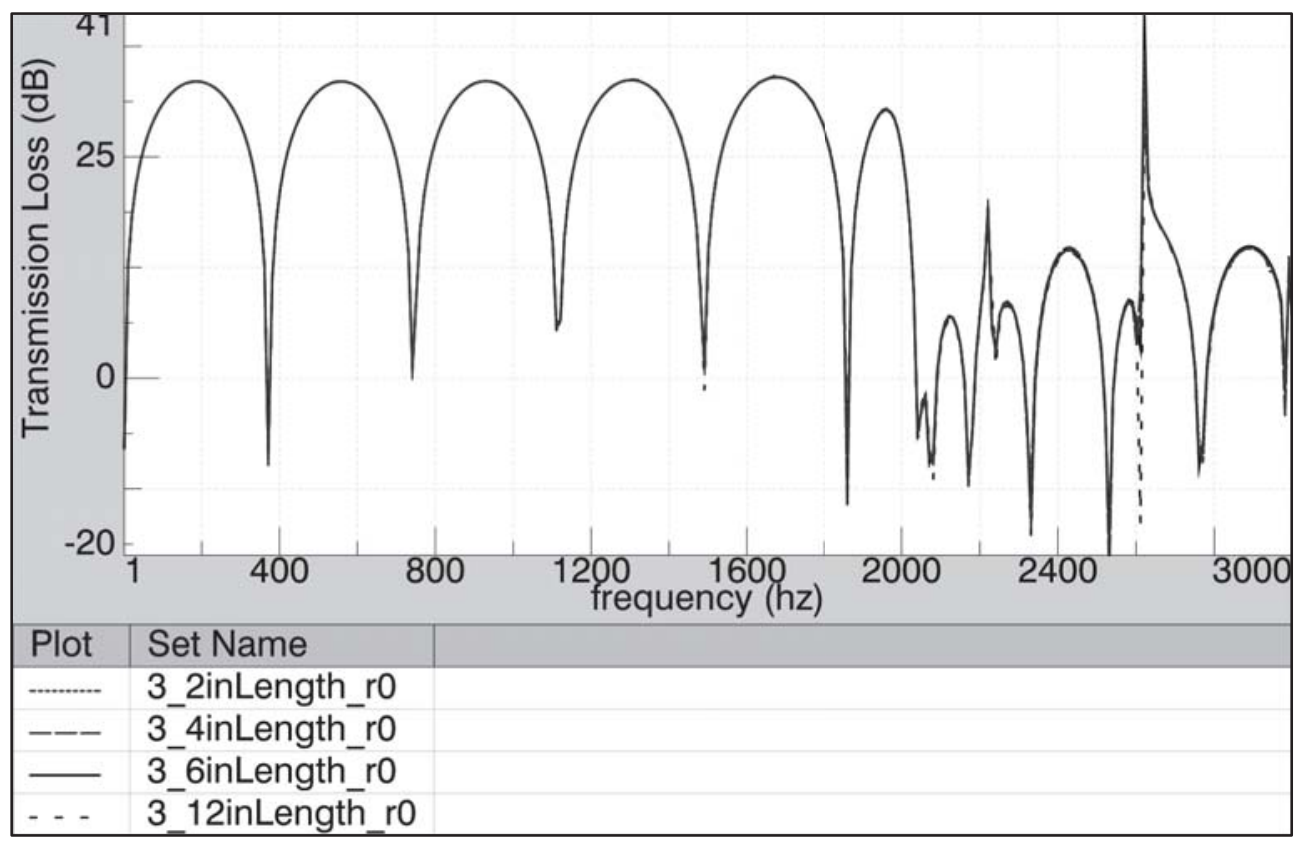

Figure 5.3: Case 3 parametric study -the length of the inlet and outlet tube are varied for the single expansion chamber. 


\subsubsection{Case 4 - Change the diameter of the inlet and outlet tube with single expansion chamber}

The next study was to change the inlet and outlet tube diameters to dimensions of 0.835", 1", and 1.5". The results are shown in Figure 5.4 which shows that the overall height of the domes is a relation between the cross sectional area of the inlet/outlet tubes and the expansion chamber. The two major advantages of decreasing the inlet/outlet tubes (rather than increasing the expansion chamber diameter) is the space savings aspect and because the 3-D wave propagation is not shifted to a lower frequency. The downside, however, is the increase in backpressure when decreasing the inlet/outlet tube diameter. This reduces the power output of the engine and, in turn, reduces the fuel economy. The designer should be aware of the maximum backpressure requirements and base the design accordingly. The other drawback is that a smaller inlet/outlet tube diameter increases the generated flow noise inside of the exhaust system.

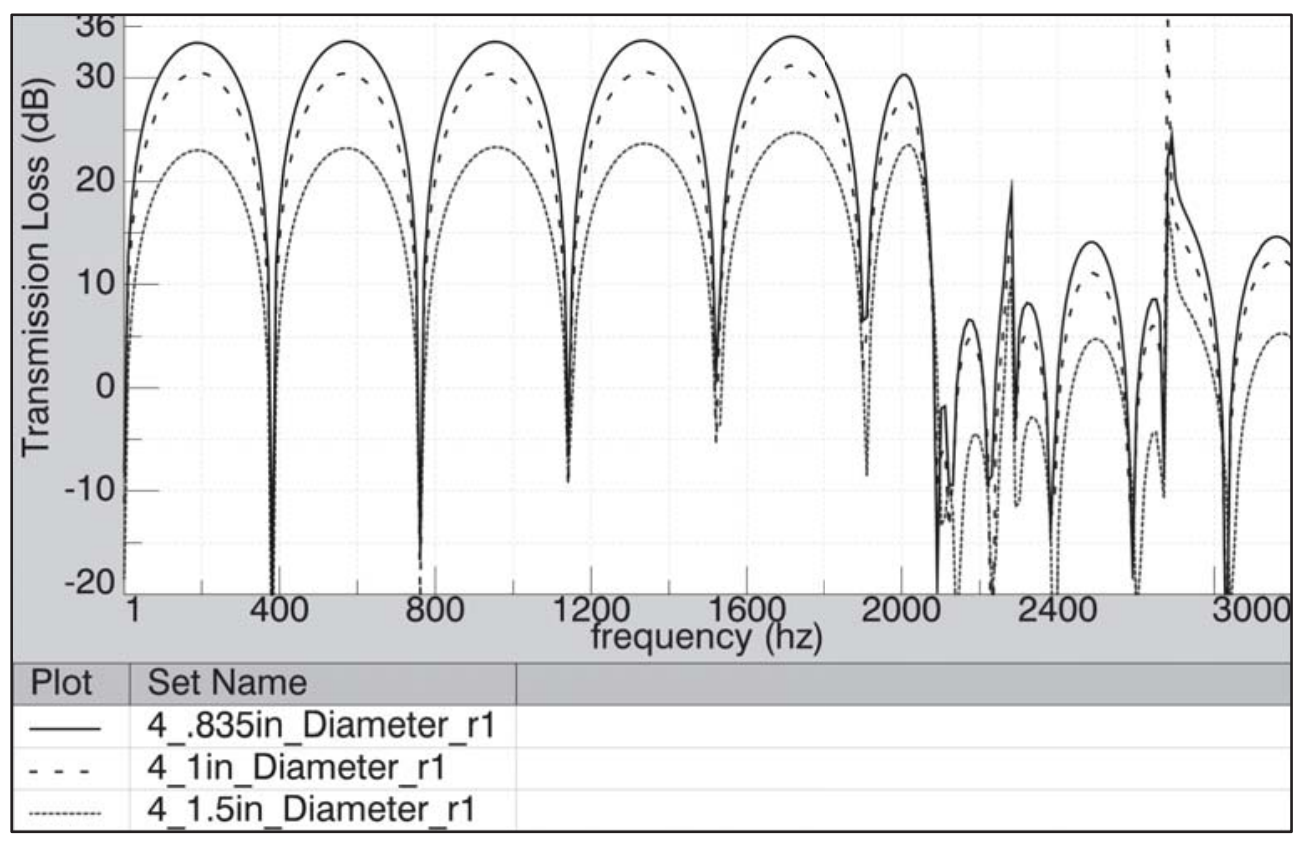

Figure 5.4: Case 4 parametric study - the diameter of the inlet and outlet tube are varied for the single expansion chamber. 


\subsubsection{Case 5 - Addition of extended tubes in single expansion chamber}

Extended tubes were added to the single expansion chamber design with lengths of 0", 0.5", 1", and 2" and the TL was calculated as shown in Figure 5.5. Seen in the figure, overall there is a slight increase in TL as a function of frequency. Typically the longer the extended tube, the higher the attenuation. This may not be true when the lengths of the extended tubes are sufficiently large when compared to the overall length of the expansion chamber. This may result in a short-circuiting effect. Because the length of the expansion chamber was relatively long for a typical exhaust system (18"), these short-circuiting effects weren't seen. The other important point is the location of the acoustical resonance the quarter wave resonators make. The location of the resonance can be calculated using Equation 2-28.

Using Equation 2-28, the only observed length that has an acoustical resonance in the frequency range of interest was for the 2" extended tube. The location of the resonance was calculated to be around $1700 \mathrm{~Hz}$. This is clearly shown in the TL as there is a drastic improvement around this frequency. It should also be noted that the acoustical resonance is spread over a $600 \mathrm{~Hz}$ bandwidth. This can be extremely useful so that the acoustical resonance of the extended tube can be felt in many operating conditions of the engine. Over the effective range of 1150 to $1900 \mathrm{~Hz}$, the 2" extended tube increased the TL, on average, by $35 \mathrm{~dB}$. For the 1" extended tube over the same bandwidth, the TL was increased by roughly $6.5 \mathrm{~dB}$. Again, the attenuation of the exhaust system is dependent on where the primary firing frequencies line up on the TL. Therefore, it is important to understand the system of interest when concluding on TL results. 


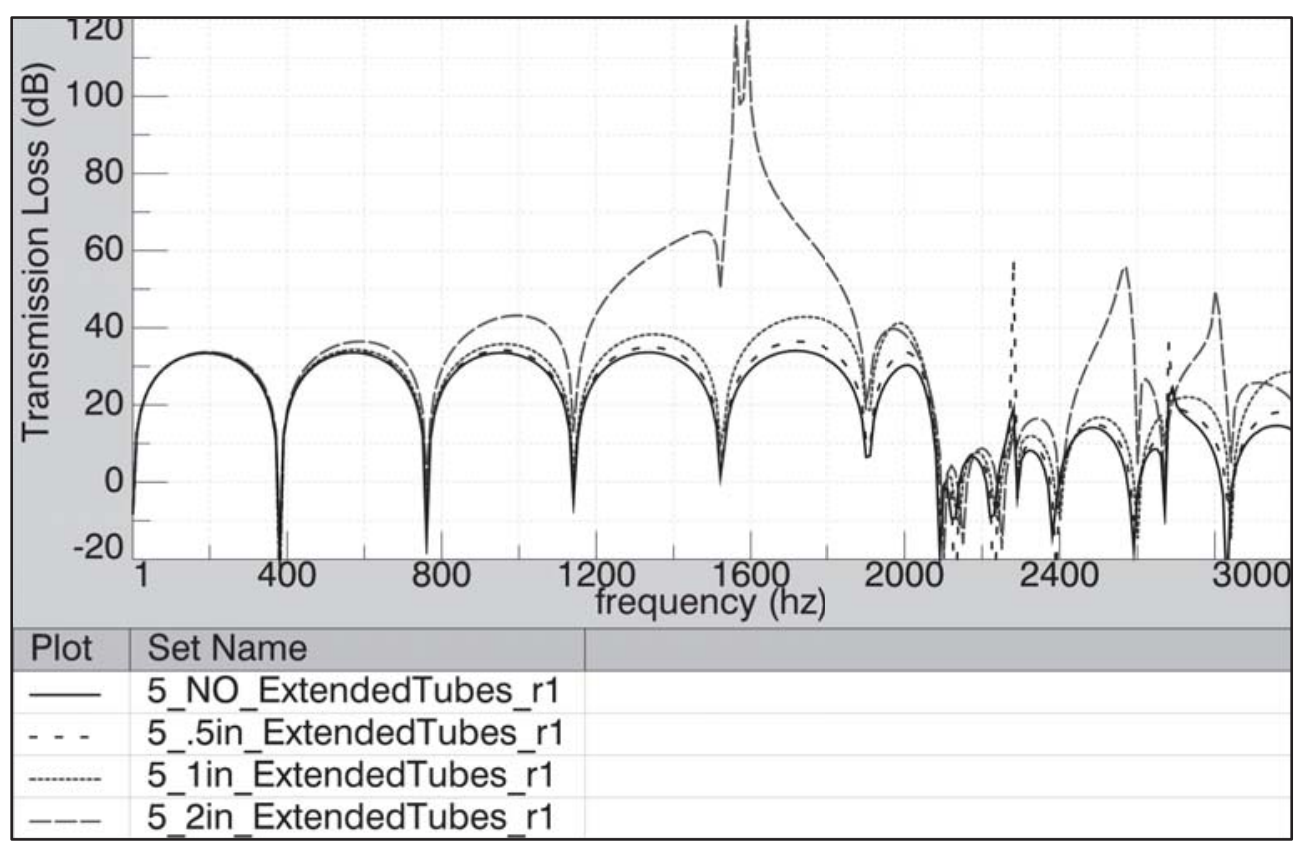

Figure 5.5: Case 5 parametric study - extended tubes are added to the single expansion chamber.

\subsubsection{Case 5.1 - Addition of perforated tubing for the single expansion chamber with 1" extended tubes}

Perforated tubing was added to the 1" extended tube single expansion chamber model. The perforated tubing had the baseline properties listed in Table 4.2 and the results for the TL are shown in Figure 5.6. The perforated tubing shows a very similar behavior on TL as did the extended tubes; there is a gradual increase in TL with an increase in frequency. The maximum increase compared to the case without perforated tubing is 4.5 and $6 \mathrm{~dB}$ for the 1 " and 2" perforated tubing, respectively. The perforated tubing is not behaving exactly like a quarterwave resonator because the flow is leaving the tubing at different locations along the pipe. Because of the flow pattern, there is no apparent acoustical resonance. A benefit of the perforated tubing is when the expansion chamber is not used effectively. The perforated tubing can be used to guide the flow path in the ideal 


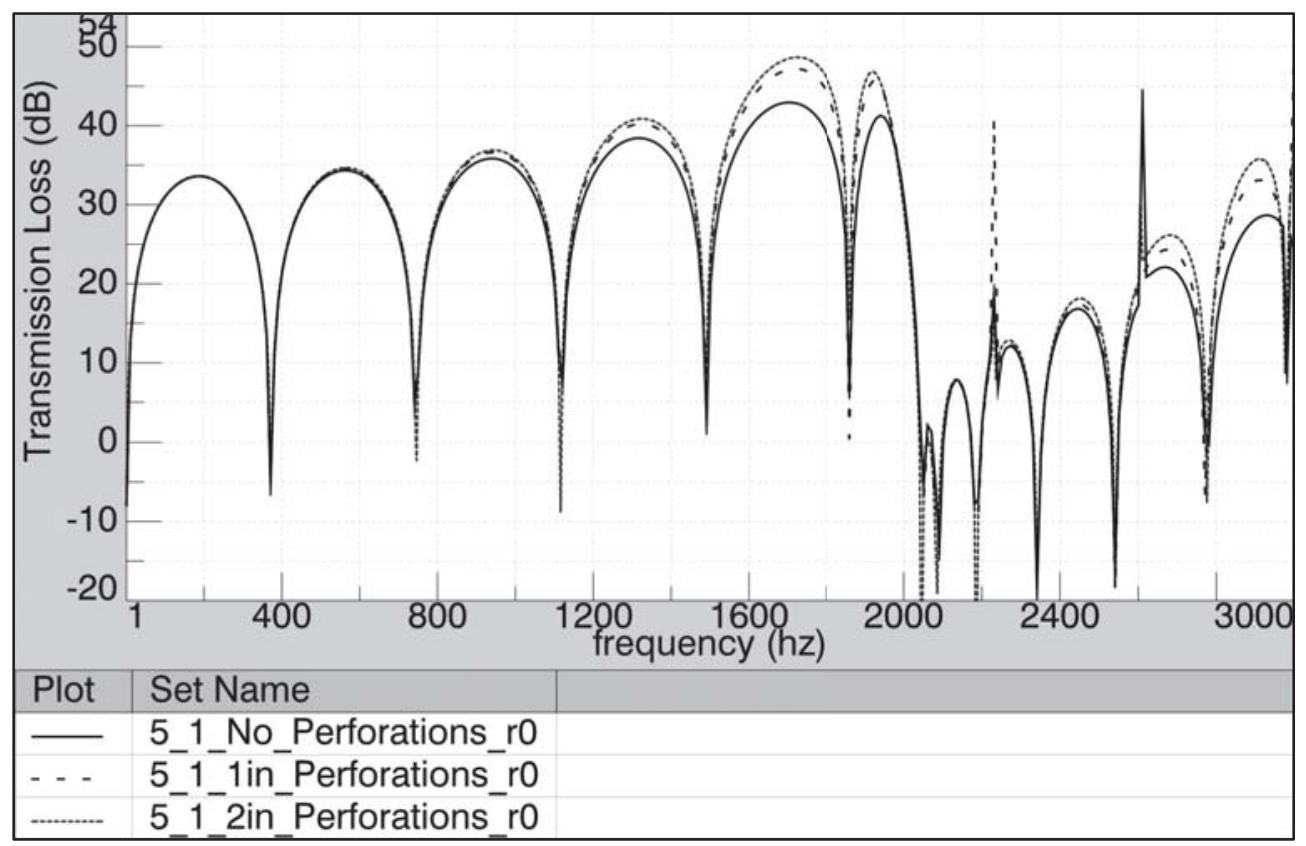

Figure 5.6: Case 5.1 parametric study - perforated tubing is added to the single expansion chamber with 1 " extended tubes.

directions to maximize the available space. This is typically the case when there is a shorter expansion length and/or high mean flow which causes a short-circuiting effect. Perforated tubing can also be used to reduce the noise generated from turbulent flow.

\subsubsection{Case 5.1.1 - Modifying the perforation properties of single expansion chamber with 1" perforated and 1" extended tubing}

The perforated tubing properties were modified for the 1" perforated, 1" extended tube single expansion chamber used in Case 5.1. The properties assigned for the three different models are described Table 4.2. The results for TL are shown in Figure 5.7. For the first property the perforated dimensions provided a very low porosity (19\%). For the second property, the porosity was increased to $43 \%$ by increasing the diameter of the holes and keeping the spacing the same. For 


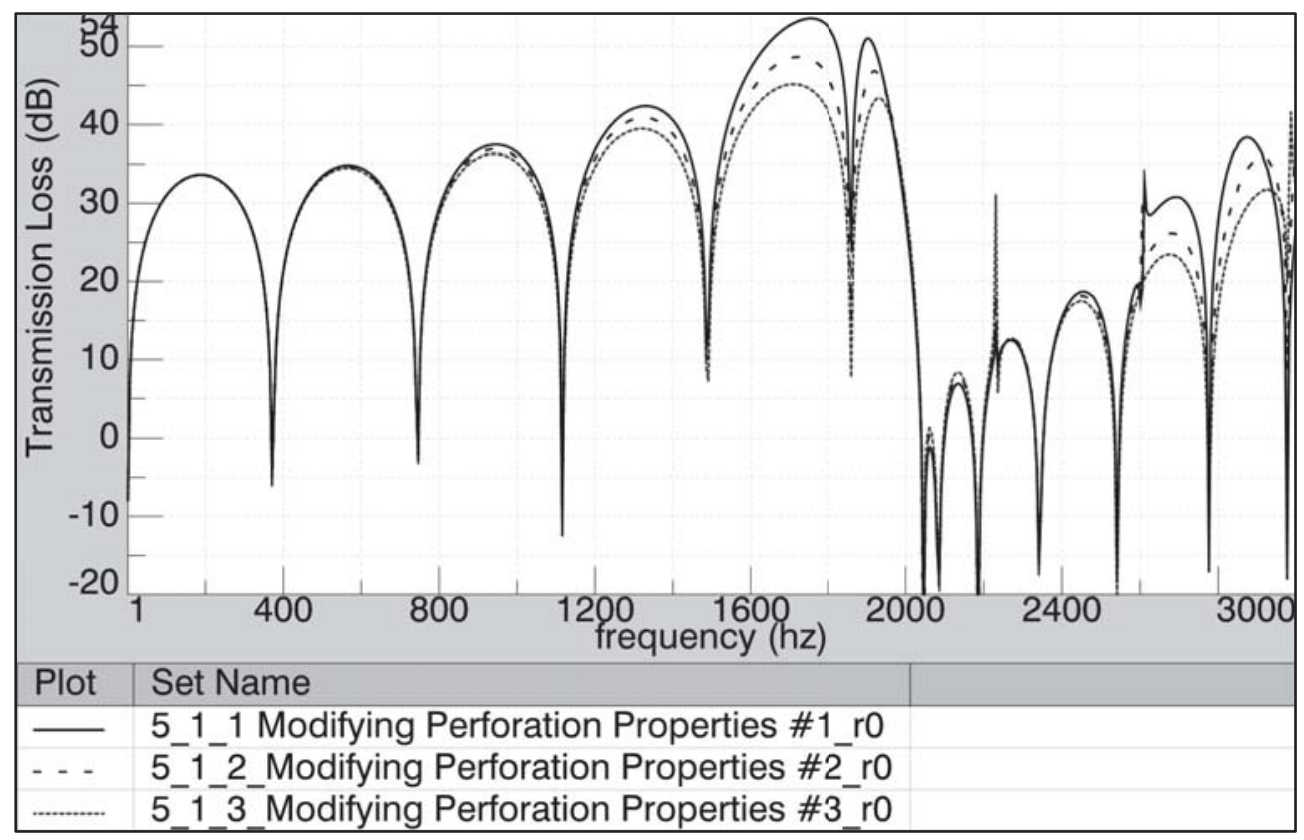

Figure 5.7: Case 5.1.1 parametric study - The perforated tubing properties were modified for the single expansion chamber with 1" perforated and 1" extended tubes.

the third property, the porosity was increased to $43 \%$ by decreasing the hole spacing and keeping the hole diameters the same.

As expected, the highest attenuation is when the porosity is the lowest because the acoustical flow is observing the most resistance. Again, the downside with choking the flow is that backpressure can be significantly affected. The designer must pay careful attention that the amount of open space is not less than the cross-sectional area of the tubing.

\subsubsection{Case 6 - Addition of baffles inside of the single expansion chamber}

Partitioning an expansion chamber is the most noteworthy way to drastically increase the maximum TL of a muffler. In this parametric study, the single expansion chamber had 0,1 , and 2 partitions placed in the expansion 
chamber and the equivalent lengths of the chambers were 18, 9, and 6 inches, respectively. Two different scenarios were calculated. First, when the partitions were infinitely thin and secondly, when the partitions were modeled as 0.5 inches. The resulting TLs for both scenarios can be seen in Figure 5.9 and Figure 5.10, respectively. The model for the three chamber design and the 0.5 " baffle is shown in Figure 5.8.

With the addition of baffles, the TL in the lower frequency spectrum is reduced while the mid to high frequency spectrum is greatly increased. This is a result of the impedance relationship between the chambers. Because of the low frequency decrease there is a clear number of expansion chambers that should be used for every scenario. Typically, most of the engine exhaust energy is contained in the first primary firing frequency which, unfortunately, is usually pretty low in the frequency spectrum. In revision $r 0$, the decision point for using 1 partition is if the primary firing frequency is above $190 \mathrm{~Hz}$ and above $275 \mathrm{~Hz}$ for 2 partitions. This would only be the case if the TL was determined with the same temperature properties seen in actual operation. Usually this is not the case, which causes the TL to shift significantly to the right. The dome widths of the TLs are governed by the length of the expansion chambers the same way as in Case 3 (the smaller the expansion chamber lengths the larger in width the domes will be).

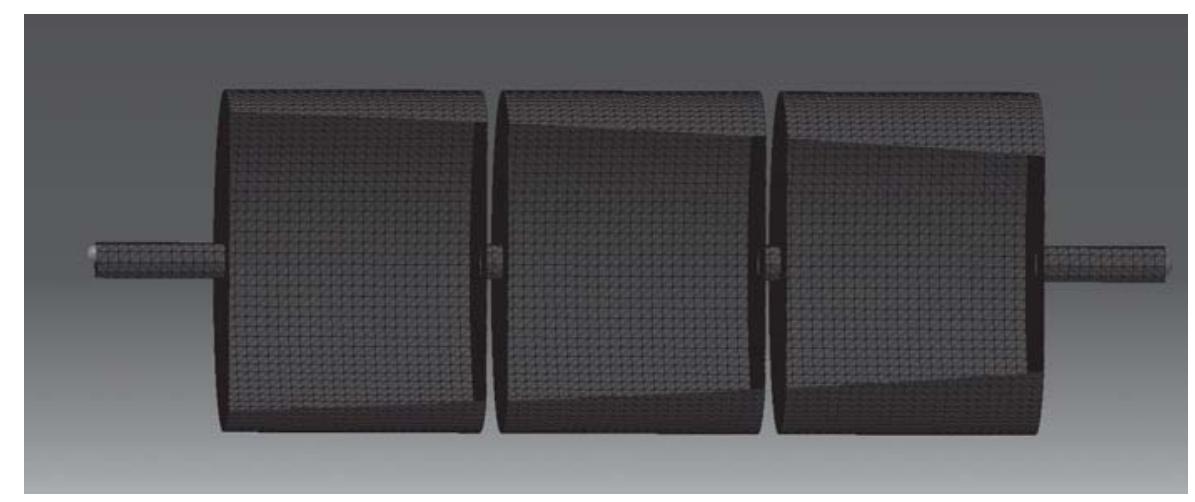

Figure 5.8: The model for the three chamber muffler with 0.5 " partitions. 
The most interesting findings from this investigation are the affects the modeled baffles have on the TL. The modeled baffle shifted the turning point frequency of the partitioned models to a lower frequency and also greatly increased the overall TL. This, again, is a result of the impedance mismatch between the two chambers. Initially there was no phase delay since the acoustical waves passed from one chamber to the next. With a baffle of finite thickness, a phase delay was imposed. There was an overall increase of $11.5 \mathrm{~dB}$ for the maximum TL of the domes and the turning point frequencies are now located at around $135 \mathrm{~Hz}$ for one partition and $195 \mathrm{~Hz}$ for two partitions.

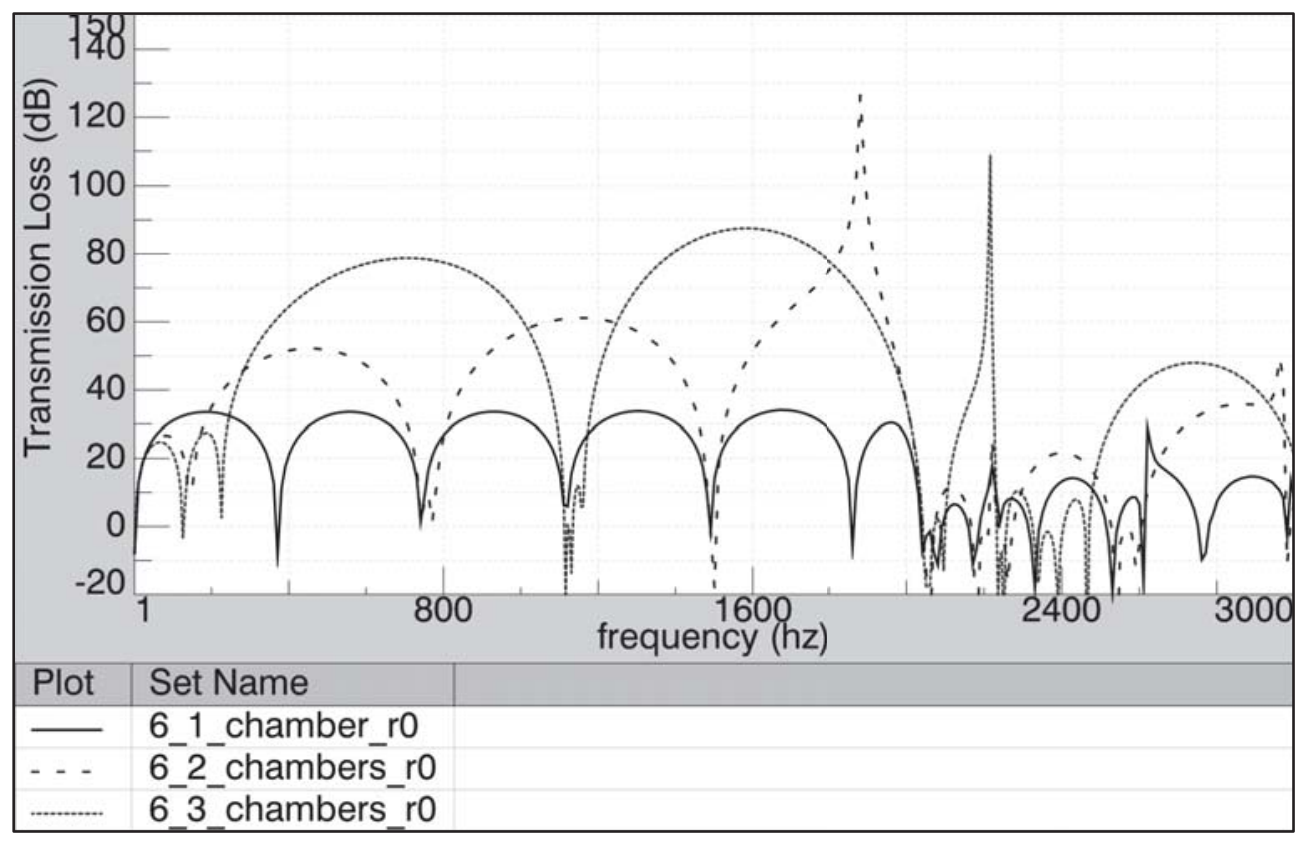

Figure 5.9: Case 6 parametric study - The single expansion chamber is split into equal length chambers where the baffle is modeled as infinitely thin. 


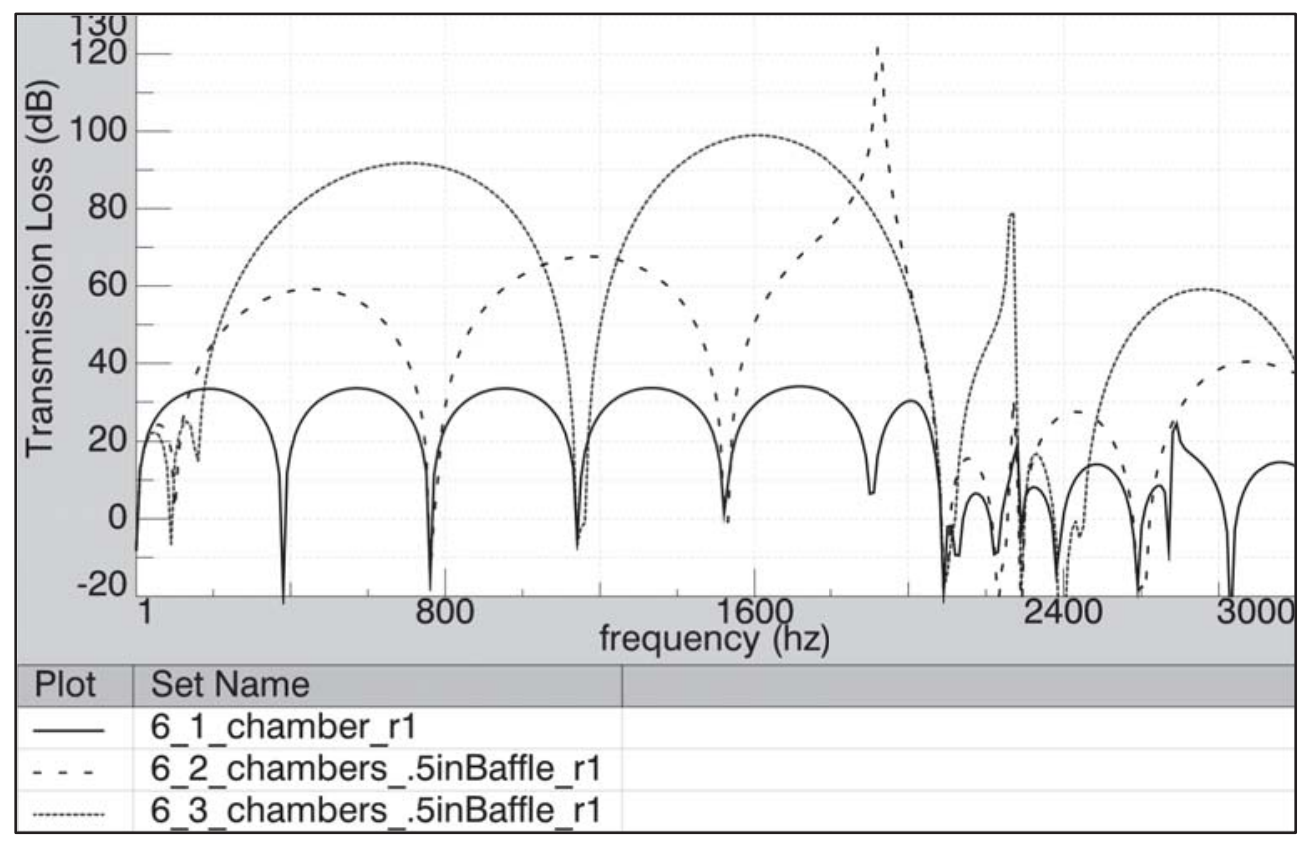

Figure 5.10: Case 6 parametric study - The single expansion chamber is split into equal length chambers where the baffle is modeled as 0.5 " thick.

\subsubsection{Case 7 - Addition of extended tubes to dual expansion chamber}

The next study performed is for the addition of extended tubes to the dual expansion chamber when the baffle is modeled as infinitely thin and with a thickness of 0.5 ". The results for the two baffle configurations with extended tube lengths of 0", 0.5", 1", and 2" are shown in Figure 5.11 and Figure 5.12, respectively. When the baffle was not modeled, there was only a minor improvement in TL with an increase in extended tube length. The addition of extended tubes resulted in about $1.5 \mathrm{~dB}$ improvement for 1 " extended tubes and 2 $\mathrm{dB}$ for 2" extended tubes. However, in the low frequency range (the first dome shape) the TL actually was reduced when the extended tubes were lengthened. The greatest reduction was for the 2" extended tubes which resulted in about a $1 \mathrm{~dB}$ drop in TL. The acoustical resonance of the 2" extended tube is present at around 
$1700 \mathrm{~Hz}$, however, the effects of the resonance is not nearly as wide or dominate as the results shown for Case 5.

When the baffle was modeled, there was another significant increase in TL and the elements played more of an impact in improving the TL of the muffler. With the addition of the 1" extended tubes the TL was increased by roughly $11 \mathrm{~dB}$ and $18 \mathrm{~dB}$ for the 2 " extended tubes. An increased length of the extended tube pushed the turning point frequency (discussed in Case 6) to the left further and also increased the overall TL. The TL for the 2" extended tube is drastically improved because of the acoustic resonance of the pipe. The acoustic resonance appears much lower in frequency than for Case 5 when the 2" extended tubes were added to the single expansion chamber. Also noted for the case with 2" extended tubes, the TL of the muffler with the properly modeled baffle is nearly $35 \mathrm{~dB}$ higher than if the baffle was not modeled.

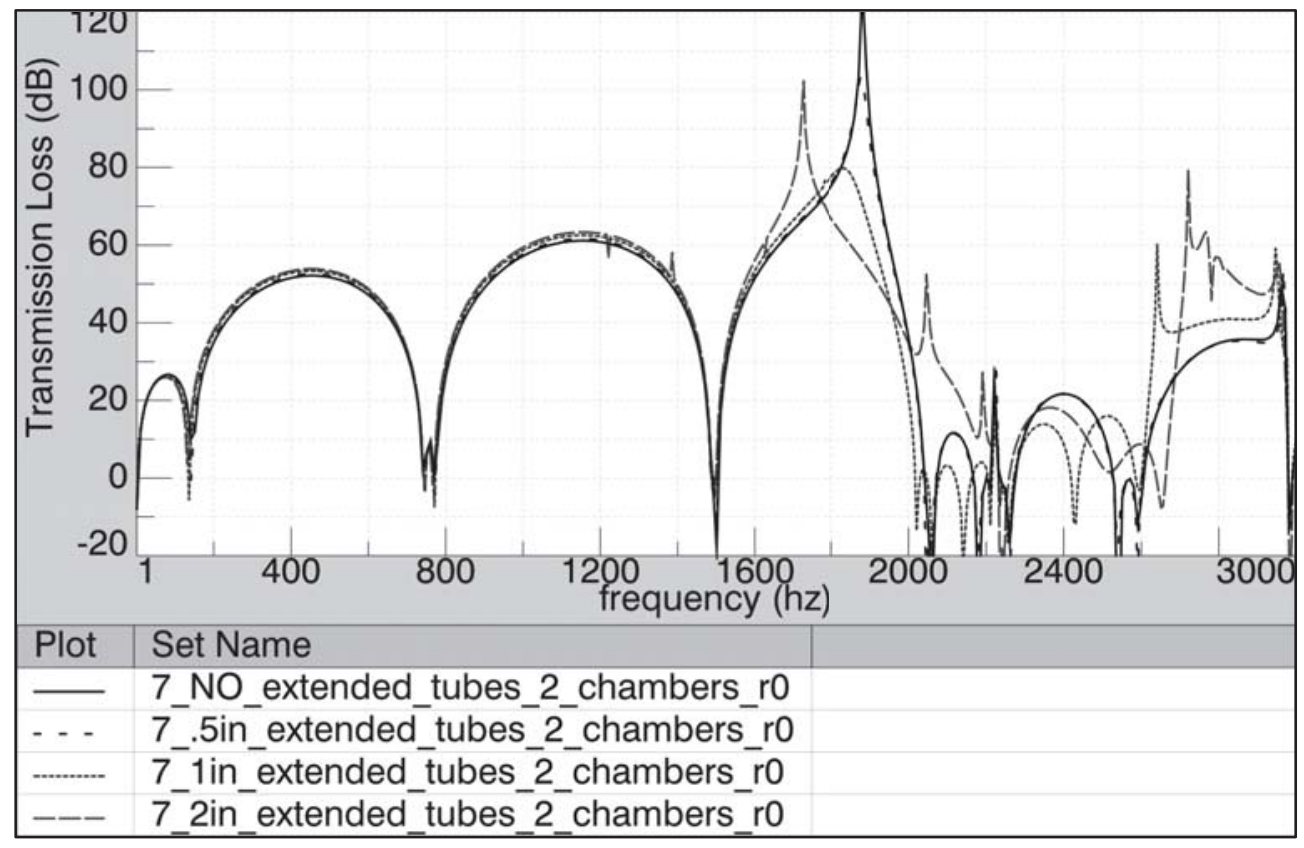

Figure 5.11: Case 7 parametric study - extended tubes are added to the dual expansion chamber where the baffle is modeled as infinitely thin. 


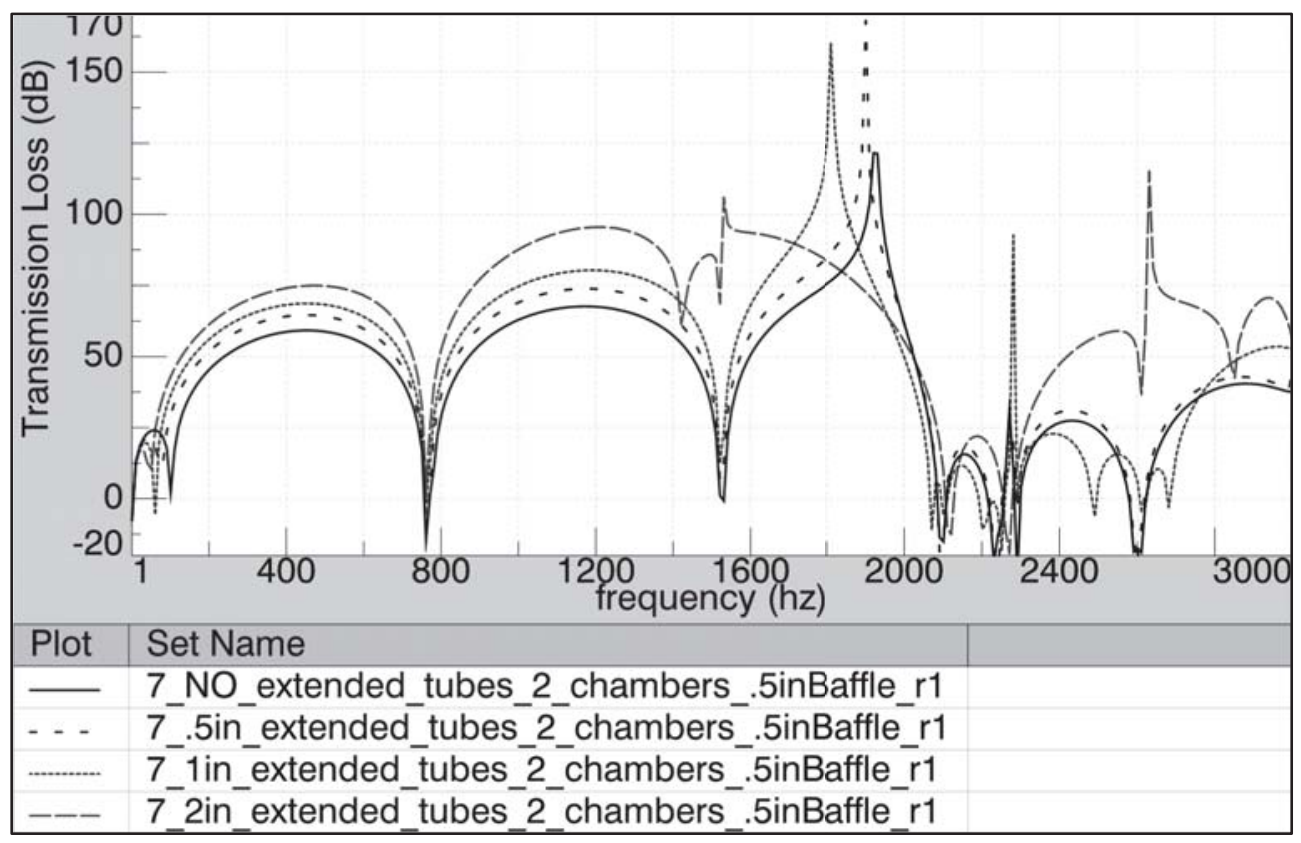

Figure 5.12: Case 7 parametric study - extended tubes are added to the dual expansion chamber where the baffle is modeled as 0.5 " thick.

\subsubsection{Case 7.1 - Addition of perforated tubing for the dual expansion chamber with 1" extended tubes}

In the next case perforated tubing with lengths of 0 ", 1", and 2" were added to the dual expansion chamber model with 1" extended tubes. The results for when the baffle is infinitely thin and modeled as 0.5 " thick are shown in Figure 5.13 and Figure 5.14, respectively. In the first case, when the baffle was not modeled, the results were virtually unaffected by the length of perforated tubing. This was not the case when the baffle was actually modeled. When the baffle was modeled, the perforated tubing had nearly the exact same effect as the extended tubes in the previous case. The addition of 1" and 2" extended tubes increased the TL by approximately 1.5 and $12.5 \mathrm{~dB}$, respectively. When the perforated tubing was 2" in length there, again, appeared to be an acoustical resonance much like the 2" 
quarter wave resonator. This was something that was not found when the 2" perforated tubing was added to the single expansion chamber.

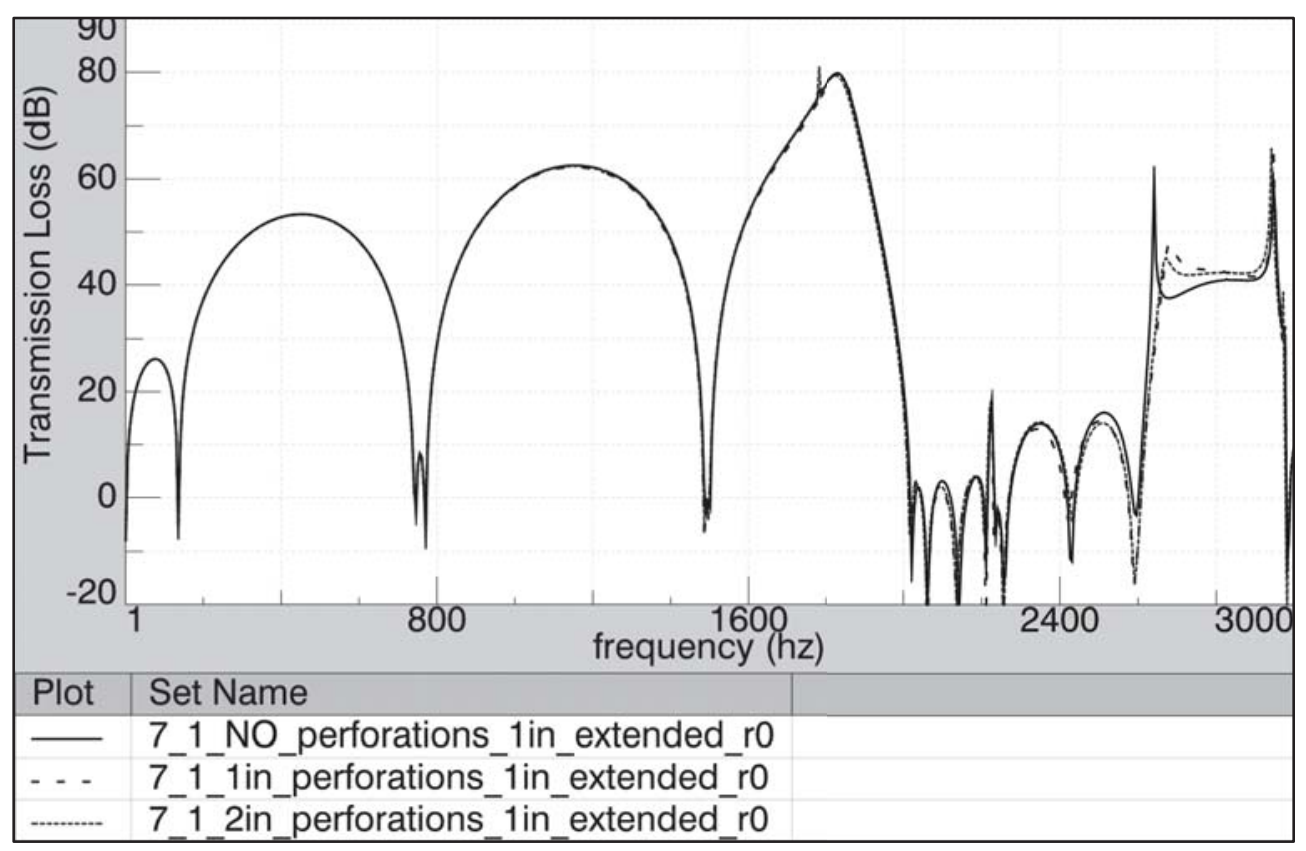

Figure 5.13: Case 7.1 parametric study -perforated tubing is added to the dual expansion chamber with 1" extended tubes. The baffle is modeled as infinitely thin. 


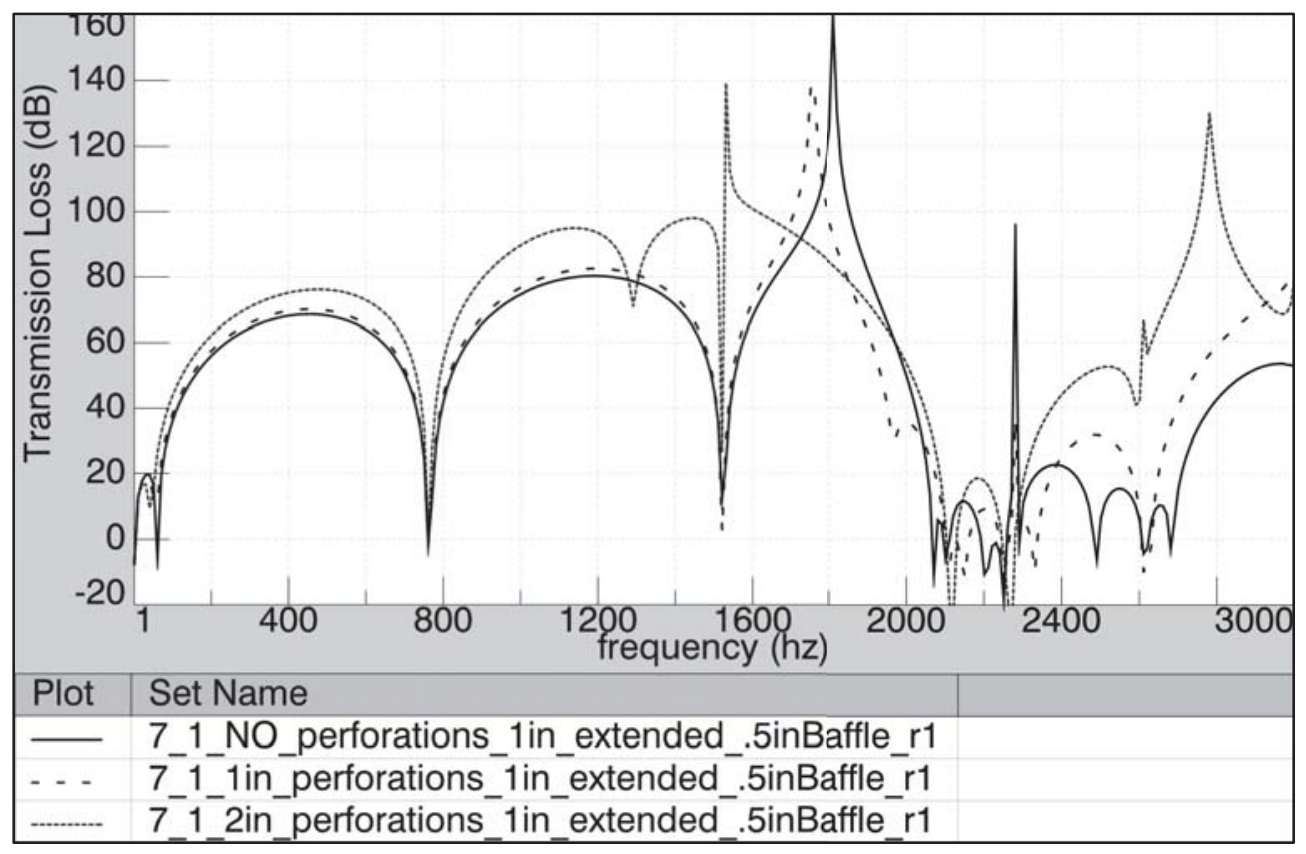

Figure 5.14: Case 7.1 parametric study -perforated tubing is added to the dual expansion chamber with 1" extended tubes. The baffle is modeled as 0.5 " thick.

\subsubsection{Case 7.1.1 - Modifying the perforation properties of single expansion chamber with 1" perforated and 1" extended tubing}

The perforated tubing properties were modified for the 1" perforated, 1" extended tube dual expansion chamber used in Case 7.1. The properties assigned for the three different models are located in Table 4.2. The results for TL when the baffle is modeled as infinitely thin and 0.5" thick is seen in Figure 5.15 and Figure 5.16. The modified perforated tube properties had marginal effects on the overall TL of the muffler. Typically, a decrease in porosity will result in an increase in TL; this is slightly more apparent when the baffle was actually modeled. 


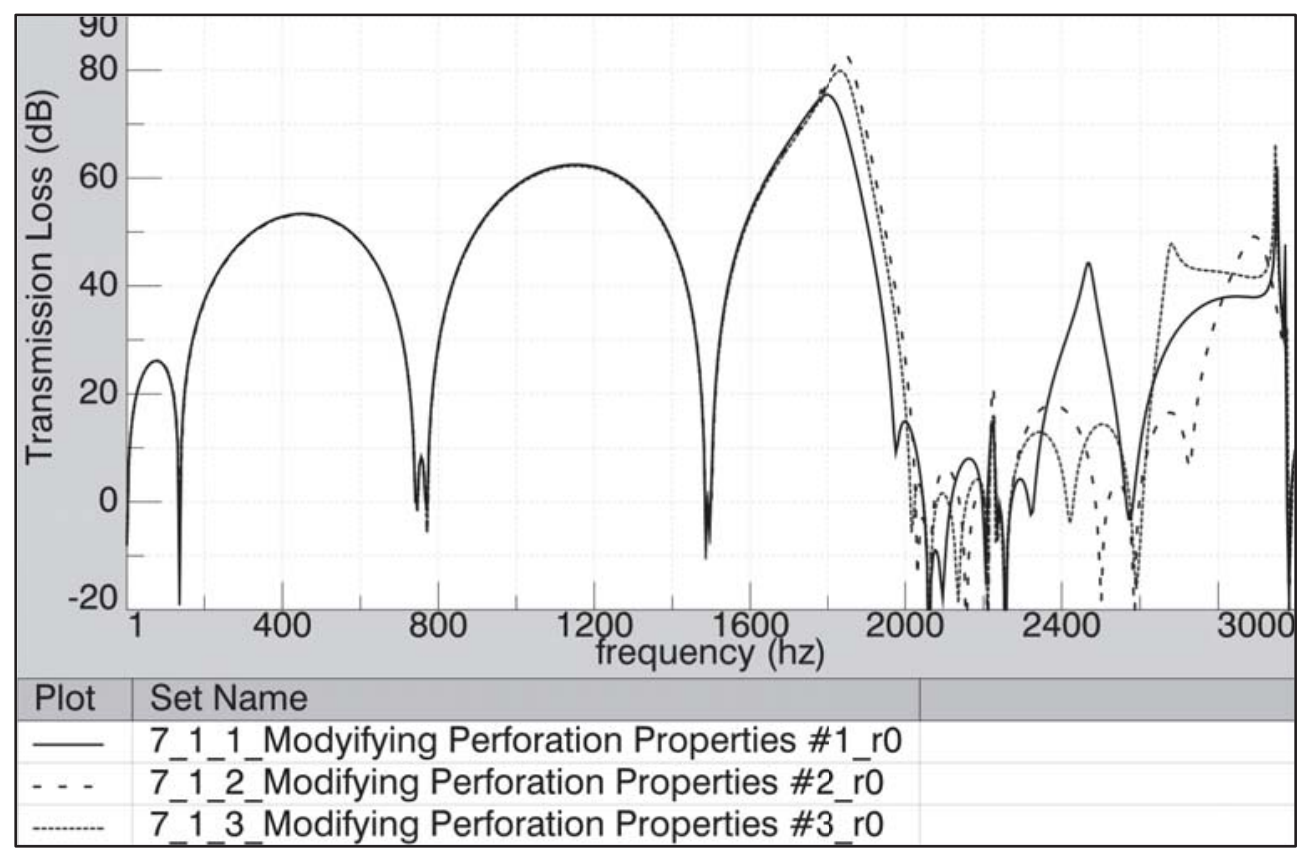

Figure 5.15: Case 7.1.1 parametric study - the perforation properties are modified for the dual expansion chamber with 1" perforated and 1" extended tubes. The baffle is modeled as infinitely thin.

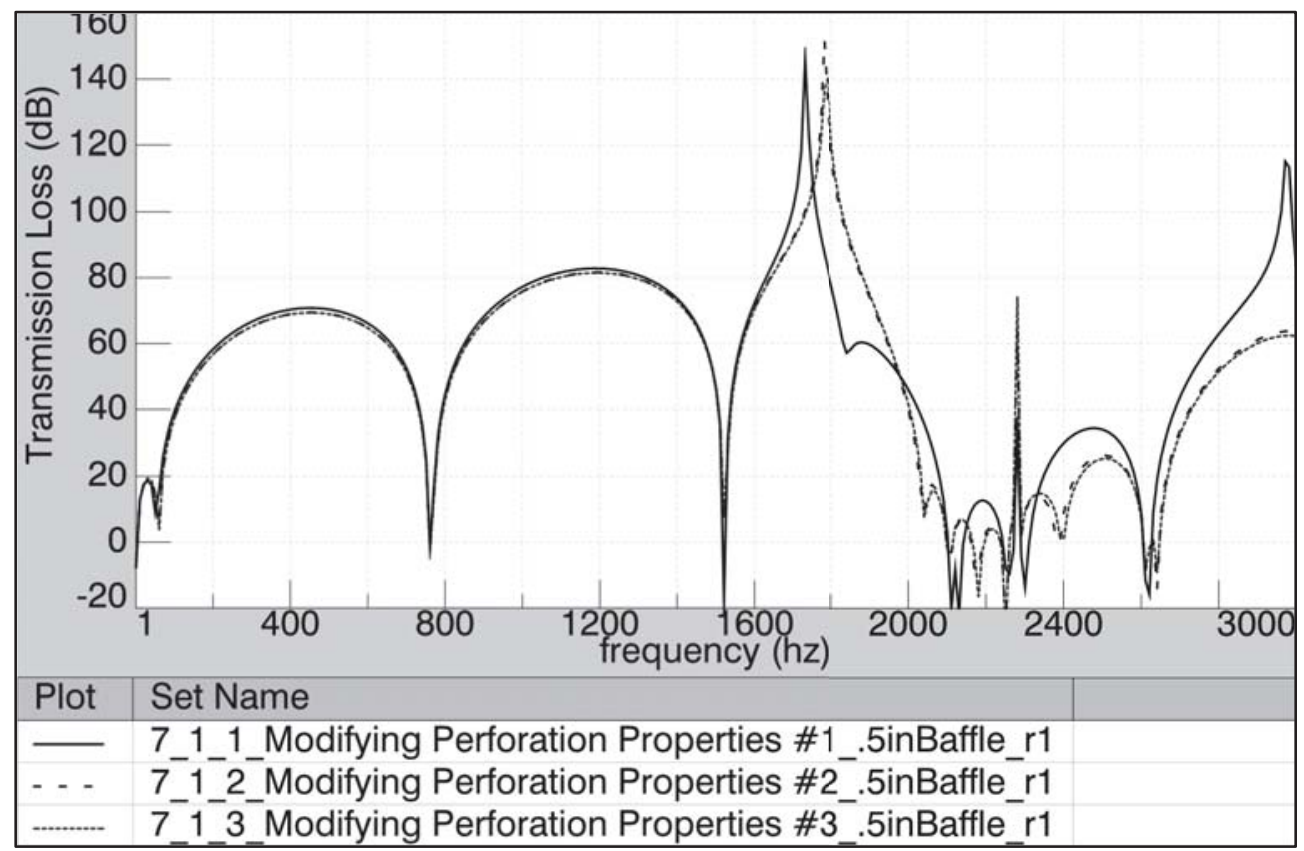

Figure 5.16: Case 7.1.1 parametric study - the perforation properties are modified for the dual expansion chamber with 1" perforated and 1" extended tubes. The baffle is modeled as 0.5 " thick. 


\subsubsection{Case 8 - Absorptive material is added to the dual expansion chamber with 1" extended tubes.}

This case involved adding the acoustic material SilcoSoft inside of the dual expansion chamber with 1" extended tubes. The acoustic material (with thicknesses of 0", $0.25 ", 0.5$ ", and 1") was added to all sides except for on the center baffle itself. The assigned properties of the material are stated in Section 3.4. A picture showing the model with the baffle modeled as 0.5 " thick and with 0.5 " material inside is shown in Figure 5.17.

The results for both cases show a general flattening of the TL with increased amount of absorption, thus reducing the peaks and increasing the troughs in the TL. Above $600 \mathrm{~Hz}$, the TL was increased by 2.5, 7, and $16 \mathrm{~dB}$ with the addition of 0.25 ", 0.5 ", and 1 " of acoustic material, respectively. The major downside of using absorptive material is that it takes up space which doesn't allow the acoustical waves to expand the entire cross-sectional area. This, in turn, reduces the low frequency attenuation of the exhaust system. Because of this, there is a finite amount of absorptive material installed which is ideal for every scenario. Below $600 \mathrm{~Hz}$, the TL was decreased by $1,2.5$, and $4.5 \mathrm{~dB}$ with the addition of 0.25 ", 0.5 ", and 1" of acoustic material, respectively.

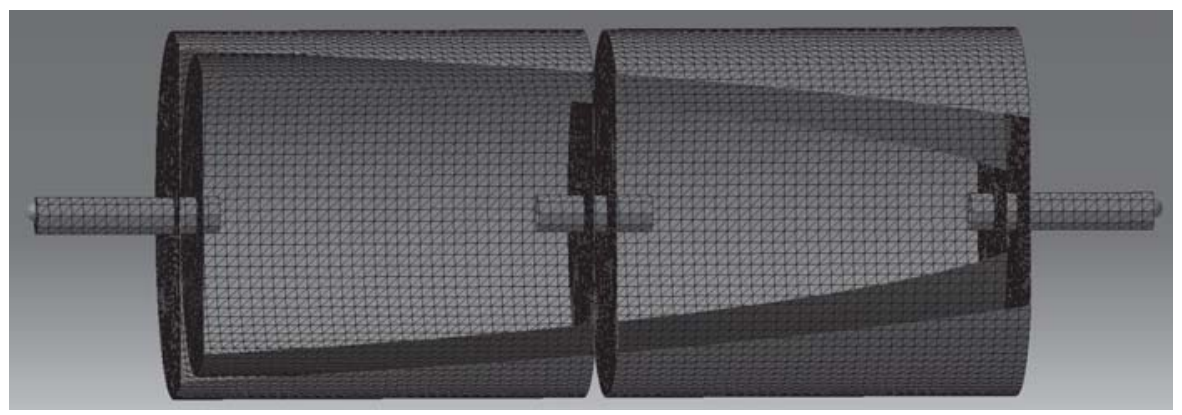

Figure 5.17: The model for the two chamber muffler with 1/2" material and 1" extended tubes. 


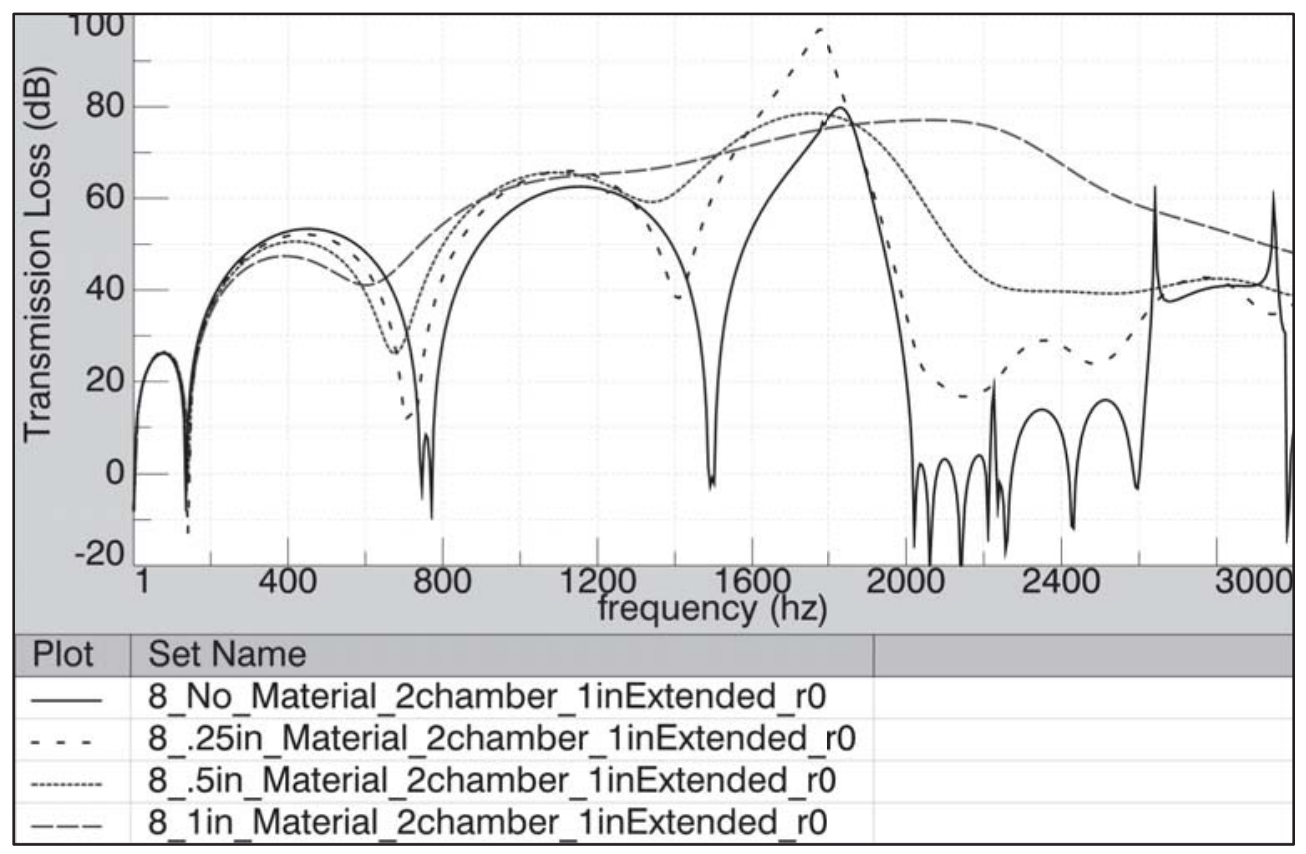

Figure 5.18: Case 8 parametric study - absorptive material is added to the dual expansion chamber with 1" extended tubes. The baffle is modeled as infinitely thin.

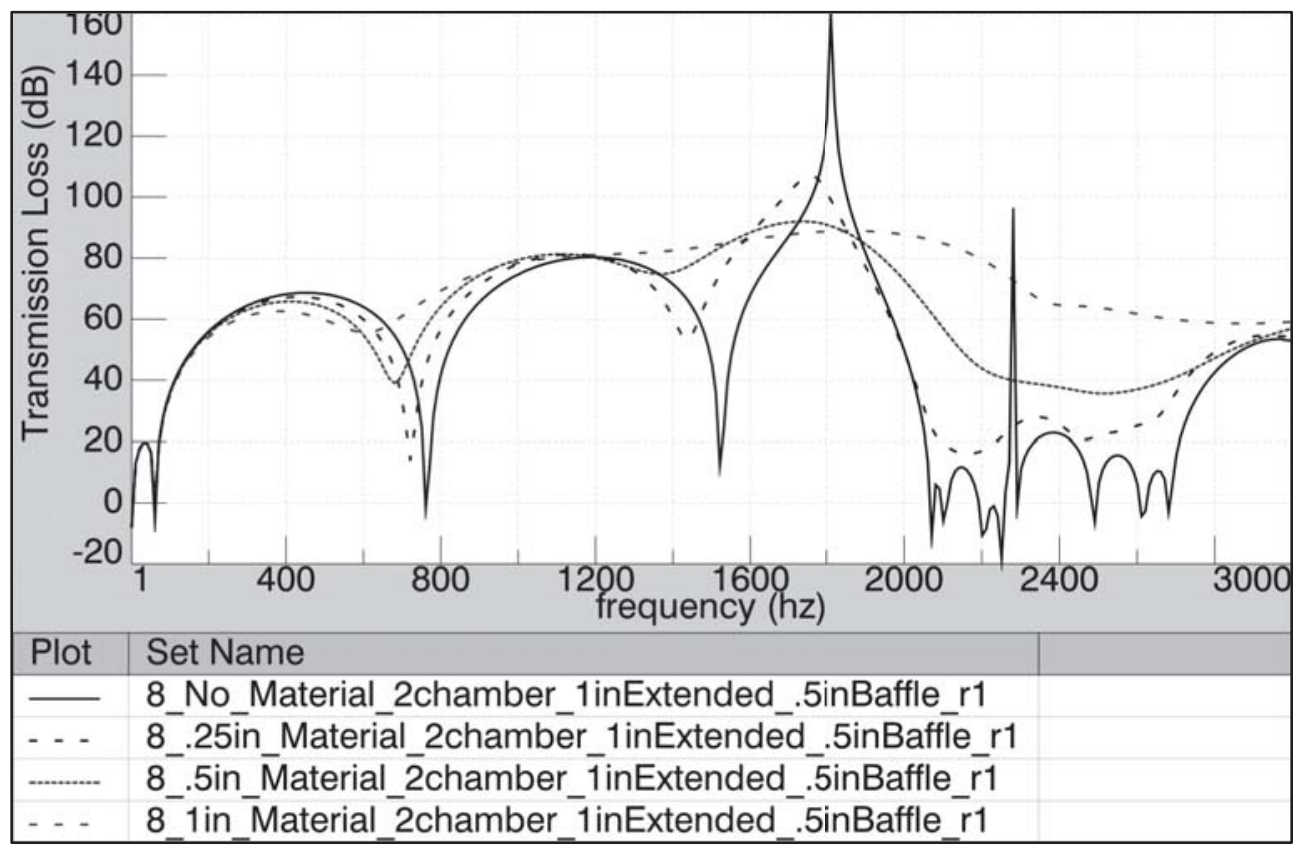

Figure 5.19: Case 8 parametric study - absorptive material is added to the dual expansion chamber with 1" extended tubes. The baffle is modeled as 0.5 " thick. 


\subsubsection{Case 8.1 - Modifying the absorptive material properties for $1 / 2$ " material dual expansion chamber with 1" extended tubes}

As the absorptive materials were approximated based on assumptions made in Section 3.4, it was necessary to vary the absorptive material properties to see their effects. The resistivity was changed by $\pm 30 \%$, the porosity was modified by $\pm 5 \%$, and the tortuosity was increased by $10 \%$ and $20 \%$. The values used for each model are presented in Table 5.1.

The resulting TLs for when the baffle is modeled as infinitely thin and 0.5 " thick are shown in Figure 5.20 and Figure 5.21, respectively. The figures show that the assumption that all woven glass fiber materials have relatively the same effect on TL holds true for this simulation. The only minor effect on TL is the resistivity of the material which is the driving parameter in determining attenuation properties of the material.

Table 5.1

The absorptive materials properties assigned to models for Case 8.1

\begin{tabular}{|c|c|c|c|}
\hline $\begin{array}{c}\text { Modification } \\
\text { Number }\end{array}$ & $\begin{array}{c}\text { Resistivity } \\
\left(\mathbf{N s} / \mathbf{m}^{\wedge} \mathbf{4}\right)\end{array}$ & Porosity & Tortousity \\
\hline Baseline & 170078 & 0.92 & 1 \\
\hline $\mathbf{1}$ & 238109 & 0.92 & 1 \\
\hline $\mathbf{2}$ & 102047 & 0.92 & 1 \\
\hline $\mathbf{3}$ & 170078 & 0.92 & 1.1 \\
\hline $\mathbf{4}$ & 170078 & 0.92 & 1.2 \\
\hline $\mathbf{5}$ & 170078 & 0.97 & 1 \\
\hline $\mathbf{6}$ & 170078 & 0.87 & 1 \\
\hline
\end{tabular}




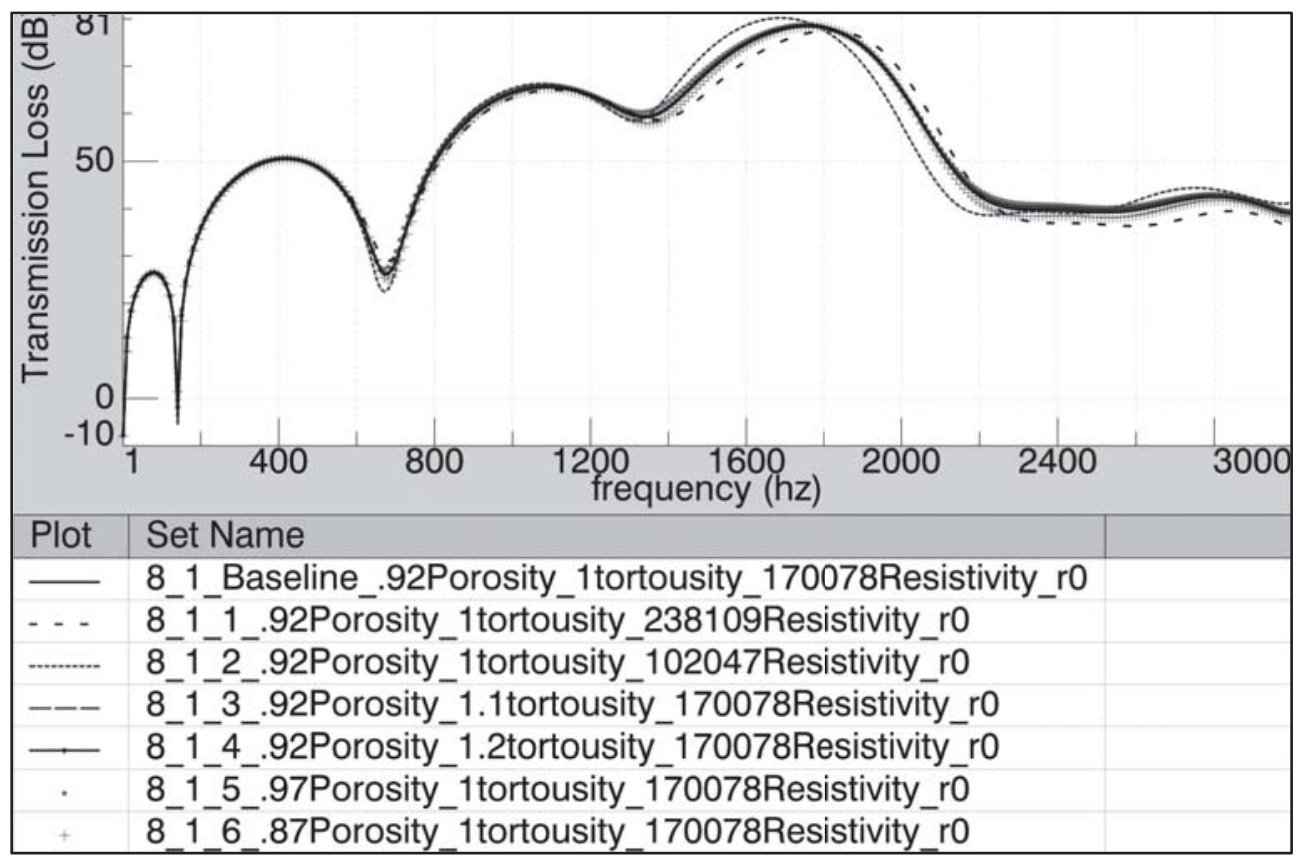

Figure 5.20: Case 8.1 parametric study - absorptive material properties are modified for the 1" material dual expansion chamber with 1" extended tubes. The baffle is modeled as infinitely thin.

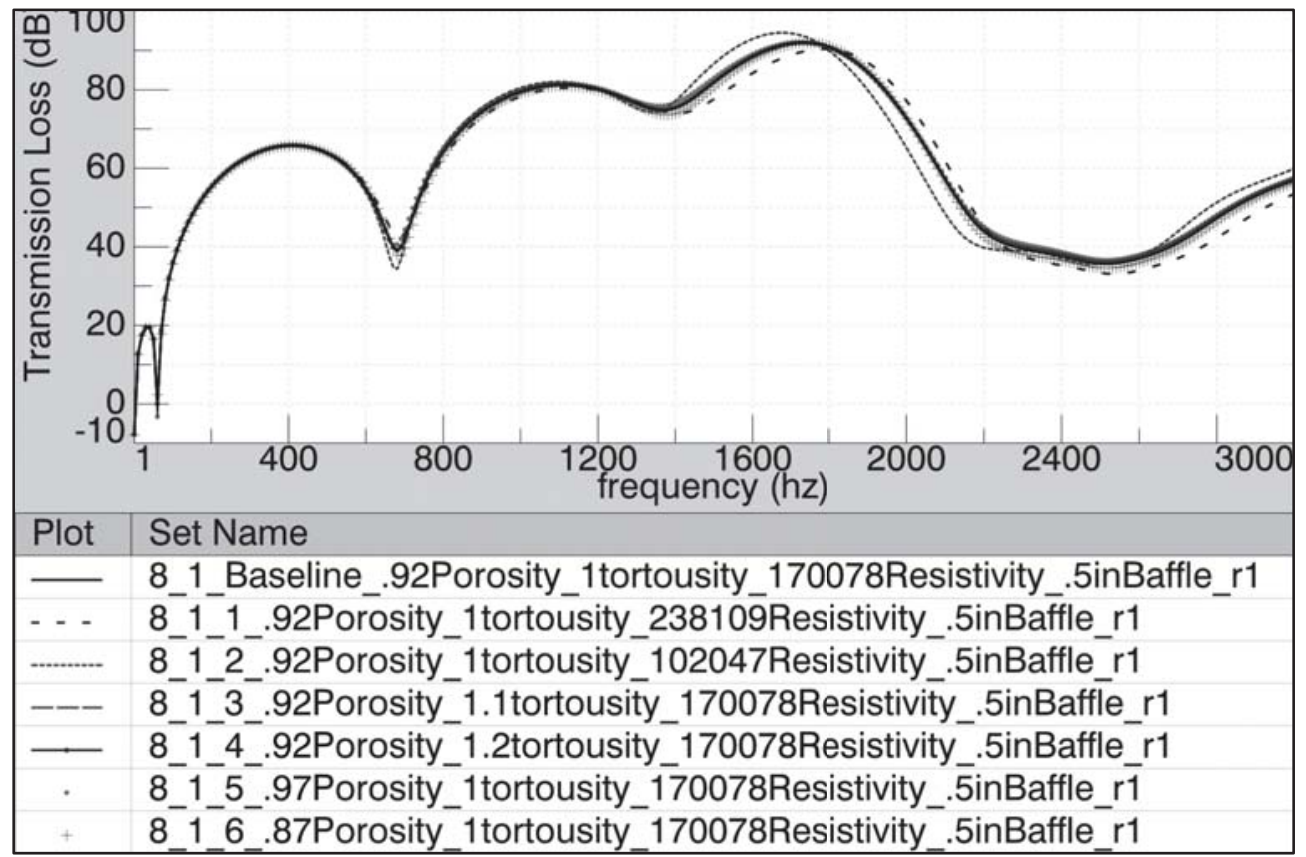

Figure 5.21: Case 8.1 parametric study - absorptive material properties are modified for the 1" material dual expansion chamber with 1" extended tubes. The baffle is modeled as 0.5 " thick. 


\subsubsection{Case 9 - Addition of mean flow through empty single expansion chamber}

In this case, mean flow through the empty single expansion chamber was examined for speeds of $0,0.05,0.15,0.25$, and $0.35 \mathrm{Ma}$. The resulting TL is shown in Figure 5.22. The simulation predicts an overall decrease in TL as a function of flow velocity. However, the overall shape of the TL is unaffected and no frequency shifting is apparent. The interesting finding is that the TL is reduced at a constant of roughly $5 \mathrm{~dB}$ for $0.35 \mathrm{Ma}$ flow at every spectral line. The effect of mean flow for this case would be equivalent to changing the inlet and outlet tube diameter which was done in Case 4.

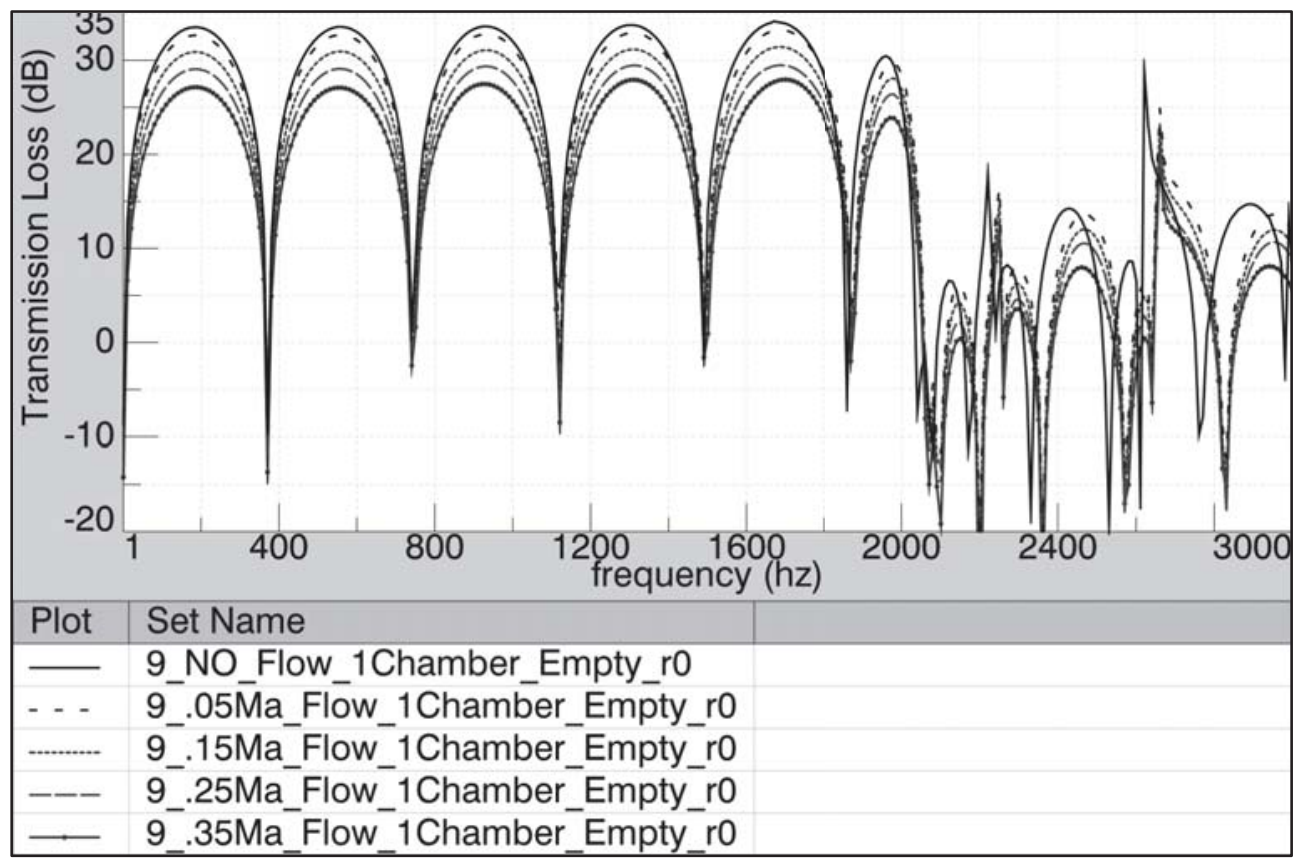

Figure 5.22: Case 9 parametric study - mean flow is incorporated into the single expansion chamber model. 


\subsubsection{Case 10 - Addition of mean flow through dual expansion chamber with 1" extended tubes}

For case 10, mean flow through the dual expansion chamber with 1" extended tubes was implemented for speeds of 0, 0.05, 0.15, 0.25, and 0.35 Ma. The resulting TL is shown in Figure 5.22. The simulation predicts an overall decrease in TL nearly identical to the values seen in Case 9. As in Case 9, the incorporation of air flow speeds of $0.05,0.15,0.25$, and 0.35 Ma reduced the TL by $0.5,2,3.5$, and $5 \mathrm{~dB}$, respectively. The findings can conclude that mean flow effects on TL using LMS's Virtual.Lab are independent of the muffler components or geometry. Intuitively, this finding is difficult to believe.

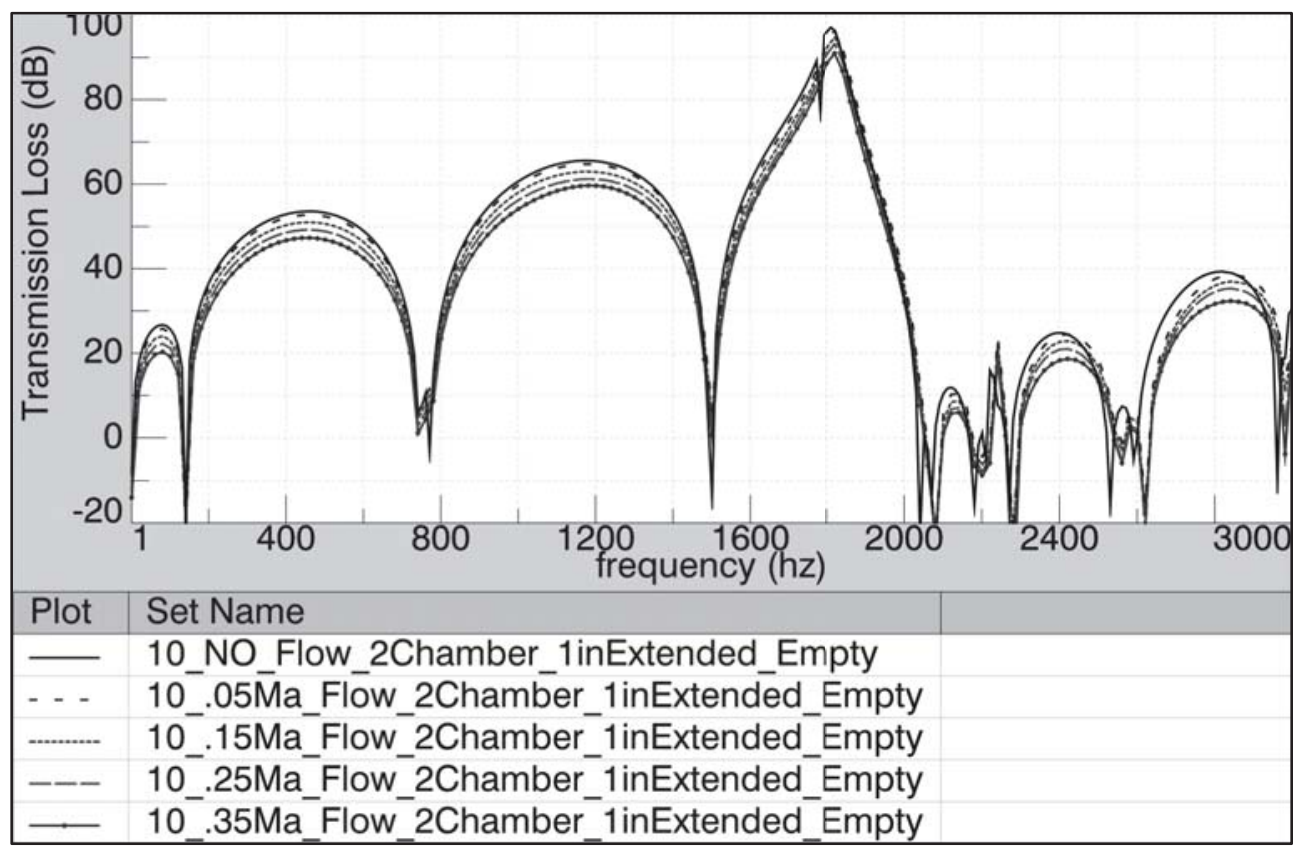

Figure 5.23: Case 10 parametric study - mean flow is incorporated into the dual expansion chamber model. The baffle is modeled as infinitely thin. 


\subsubsection{Case 11 - Temperature effects on dual expansion chamber with 1" extended tubes}

The last parametric study was to understand the effects of temperature on TL when the temperature is constant throughout the exhaust system. The density and speed of sound were determined using Equation 2-38 and Equation 2-40 at temperatures of $20,100,200$, and $400{ }^{\circ} \mathrm{C}$. The acoustic fluid properties were assigned to the dual expansion chamber with 1" extended tubes and the TL was calculated for each scenario. The results are seen in Figure 5.24 which show a shift in TL as described using Equation 2-41. This equation only holds true if the temperature inside of the exhaust system is constant. This typically is not the case in actual operation. For TL in operating condition, a temperature gradient needs to be applied to the model. This can be done by determining the temperature at every nodal point using CFD or by breaking up the muffler into elements and assigning values determined experimentally.

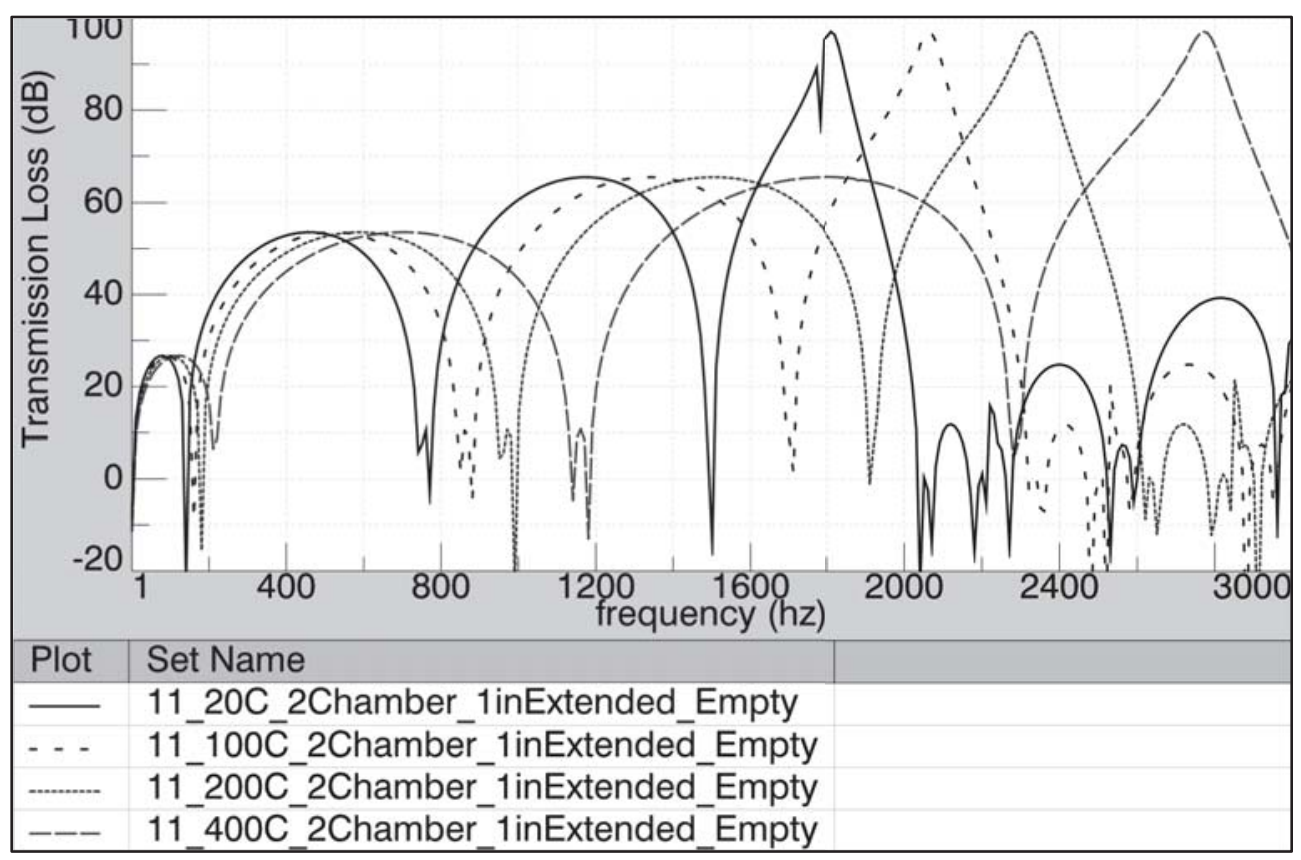

Figure 5.24: Case 11 parametric study - temperature effects are applied to the dual expansion chamber model. The baffle is modeled as infinitely thin. 


\subsection{Correlated results}

In order to build confidence in the simulated models created as well as learn experimental techniques, a correlation study was necessary. Prototypes were developed for a few of the simulation models used in the parametric study and experimental TLs were determined. The models that were developed and tested are underlined in Table 4.1.

\subsubsection{Correlation 1 - Eight inch diameter empty muffler}

The experimental results for the 8" diameter empty muffler are nearly identical to the simulation results when the simulation was updated with correct fluid properties. The simulation results for when $c=340 \mathrm{~m} / \mathrm{s}$ and $c=348 \mathrm{~m} / \mathrm{s}$ along with the experimental results are shown in Figure 5.25.

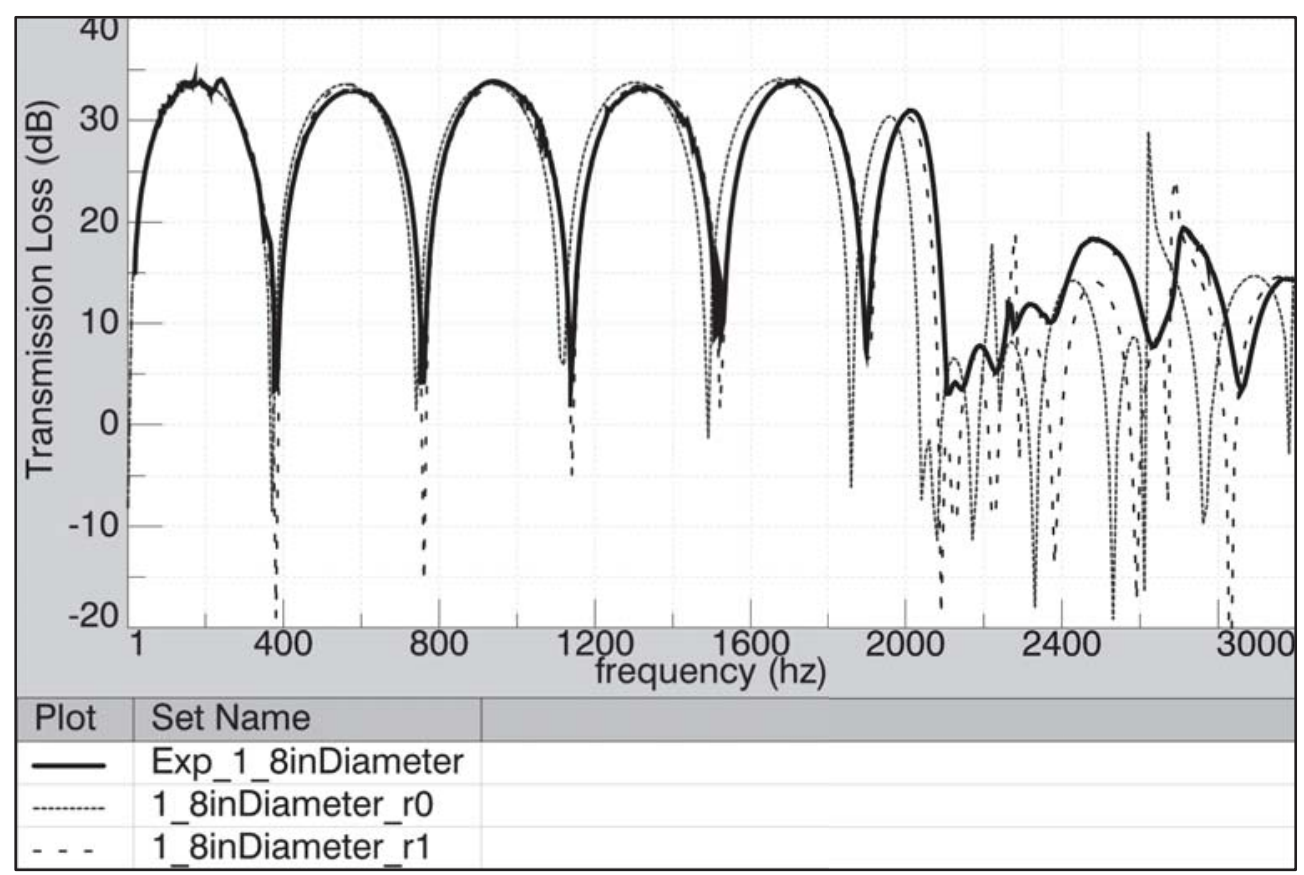

Figure 5.25: Case 1 correlation study - Eight inch diameter empty muffler 


\subsubsection{Correlation 5 - Extended tubes added to eight inch diameter muffler}

An experimental model was developed for the single expansion chamber with 1" and 2" extended tubes. The correlated TL results for both models are shown in Figure 5.26. The 1" extended tube results match fairly well, although the simulated results slightly under predicted. Both the experimental and simulated results show an acoustical resonance behavior around $1600 \mathrm{~Hz}$. It appears that the TL for the simulation results is nearly mirrored around this point.

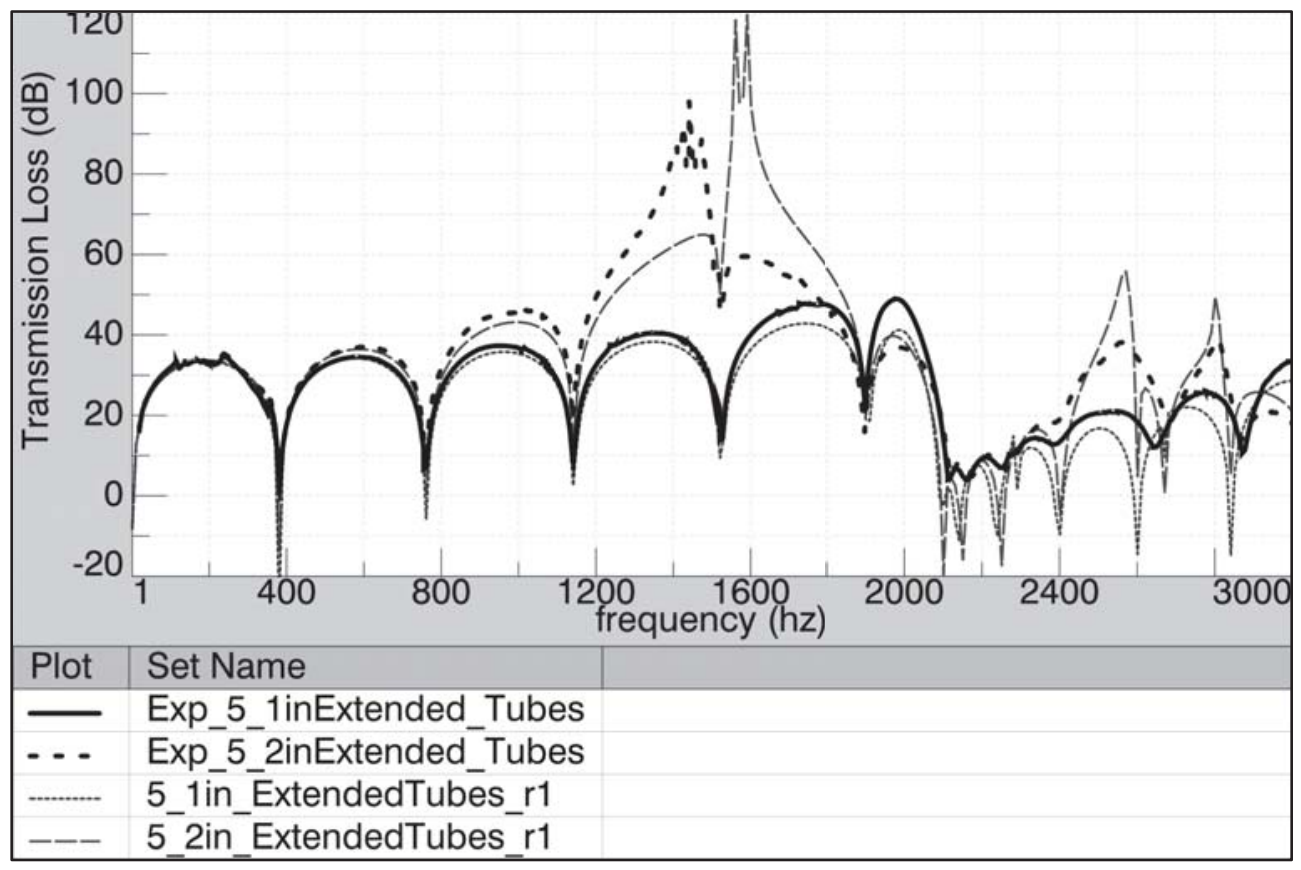

Figure 5.26: Case 5 correlation study - 1" and 2" extended tubes added to eight inch diameter muffler 


\subsubsection{Correlation 5.1 - 1 inch perforated tubing with 1 inch extended tubes added to the single expansion chamber}

The experimental model with 1" perforated tubing showed very accurate correlation to the simulated model. The results of the correlation are shown in Figure 5.27.

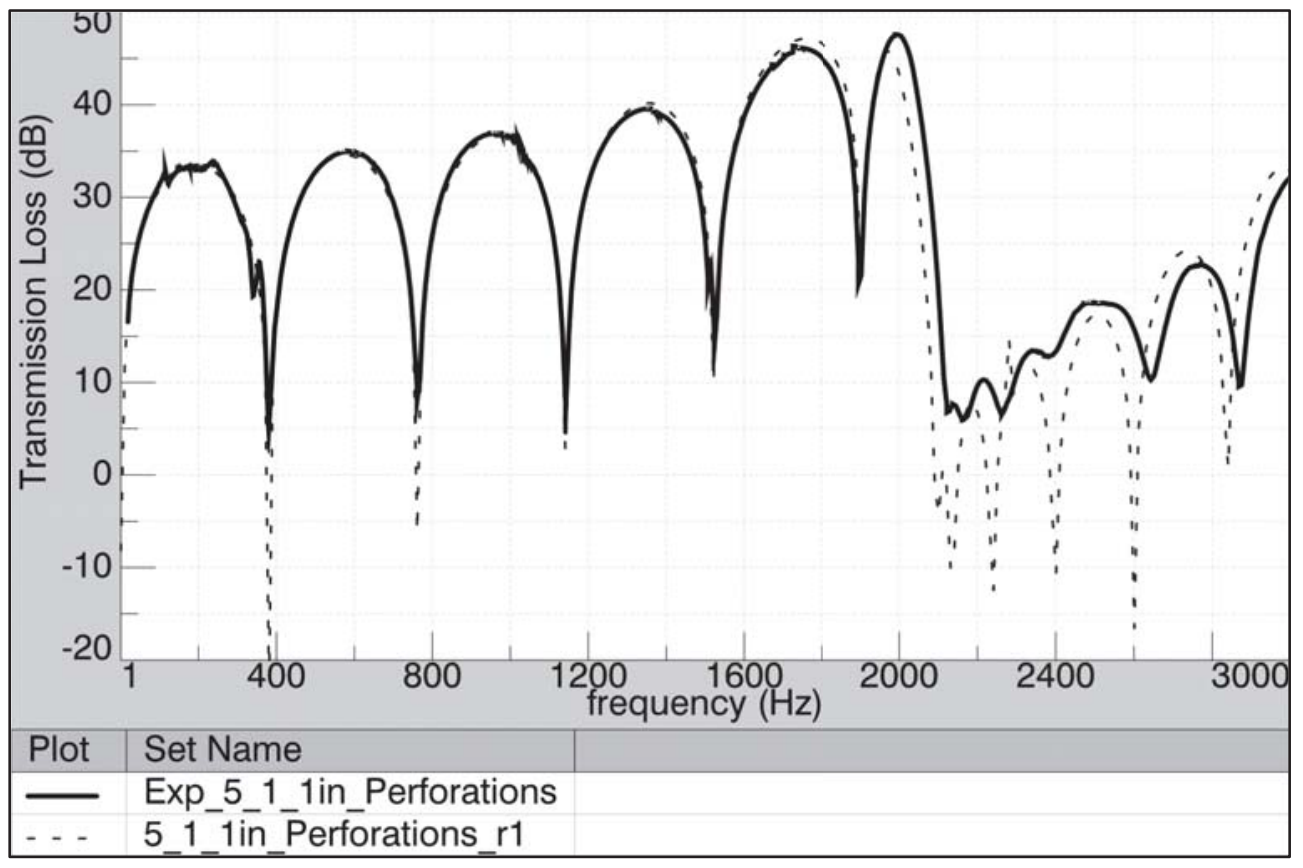

Figure 5.27: Case 5.1 correlation study - 1" perforated and 1" extended tubes in single expansion chamber. 


\subsubsection{Correlation 6 - Dual expansion chamber muffler}

The experimental model for the dual expansion chamber used a 1/2" plywood baffle as the partition. Two simulation models were created, one consisting of an infinitely thin baffle (shown as dotted line) and one consisting of a 0.5 " baffle (shown as dashed line). The correlation results can be seen in Figure 5.28. The experimental model matches the simulated model with the 0.5 " baffle very well. The width of the baffle plays a significant role in the TL of the muffler.

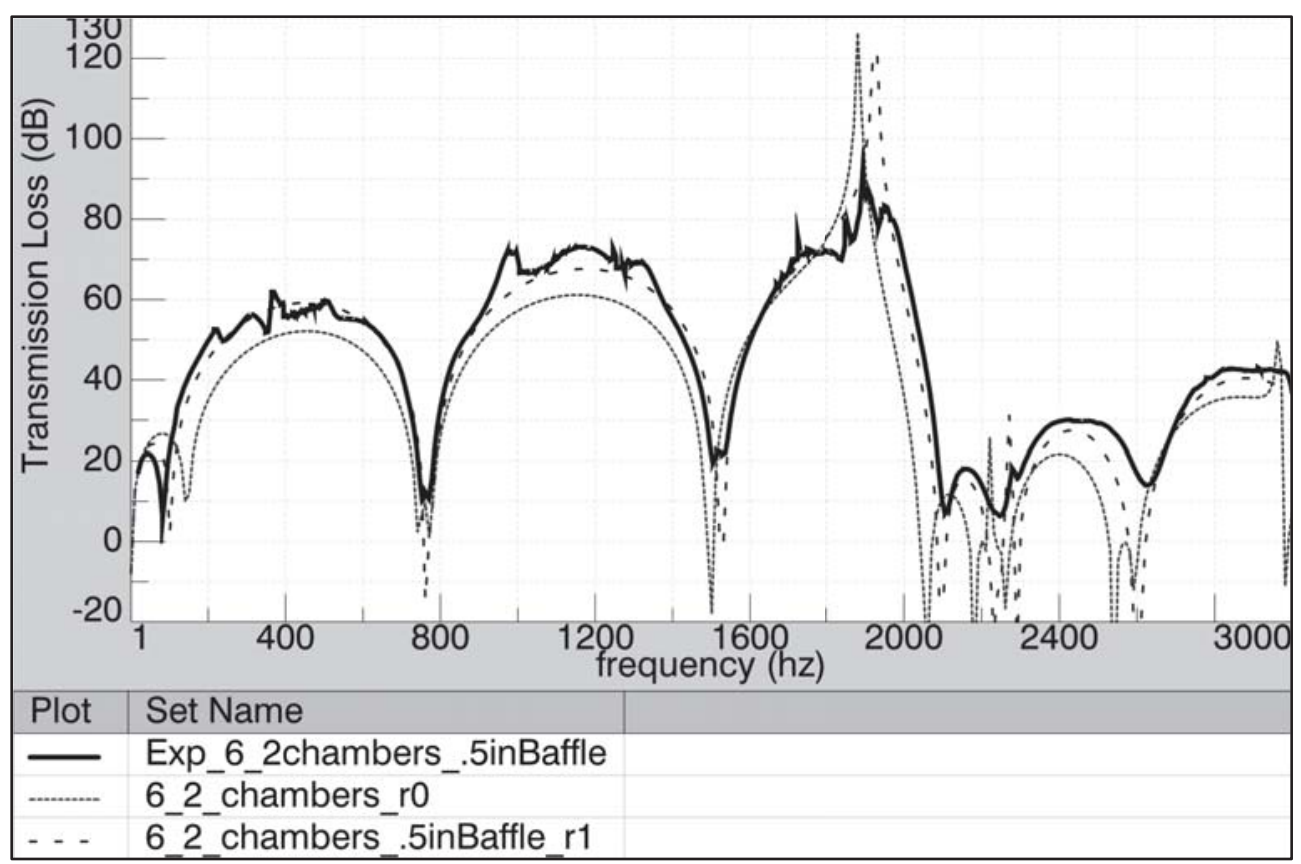

Figure 5.28: Case 6 correlation study - dual expansion chamber muffler 


\subsubsection{Correlation 7 - 1 " extended tubes added to dual expansion chamber}

The next correlation study was for the dual expansion chamber with one inch extended tubes. The results from the experimental and simulation testing are displayed in Figure 5.29. Overall, the experimental model shows a high level of correlation. However, it does appear that some additional dynamics are taking place. The dynamics taking place in the low to mid frequency range (between 200 to $1500 \mathrm{~Hz}$ ) are thought to have been caused by inaccuracies in the experimental model. One difficulty with the addition of the baffle was to provide adequate seals with no crevices between the baffles and expansion chamber. This wasn't as significant when only one chamber was used. Other possibilities for these differences are that the extended tubes may not have been perfectly in-line in the horizontal direction as well as imperfections of the baffle surfaces. Not providing enough signal in the microphones downstream of the muffler was the key contributor in the frequency range of $1600-2000 \mathrm{~Hz}$ and may have been a slight contributor in the mid to low frequency range as well.

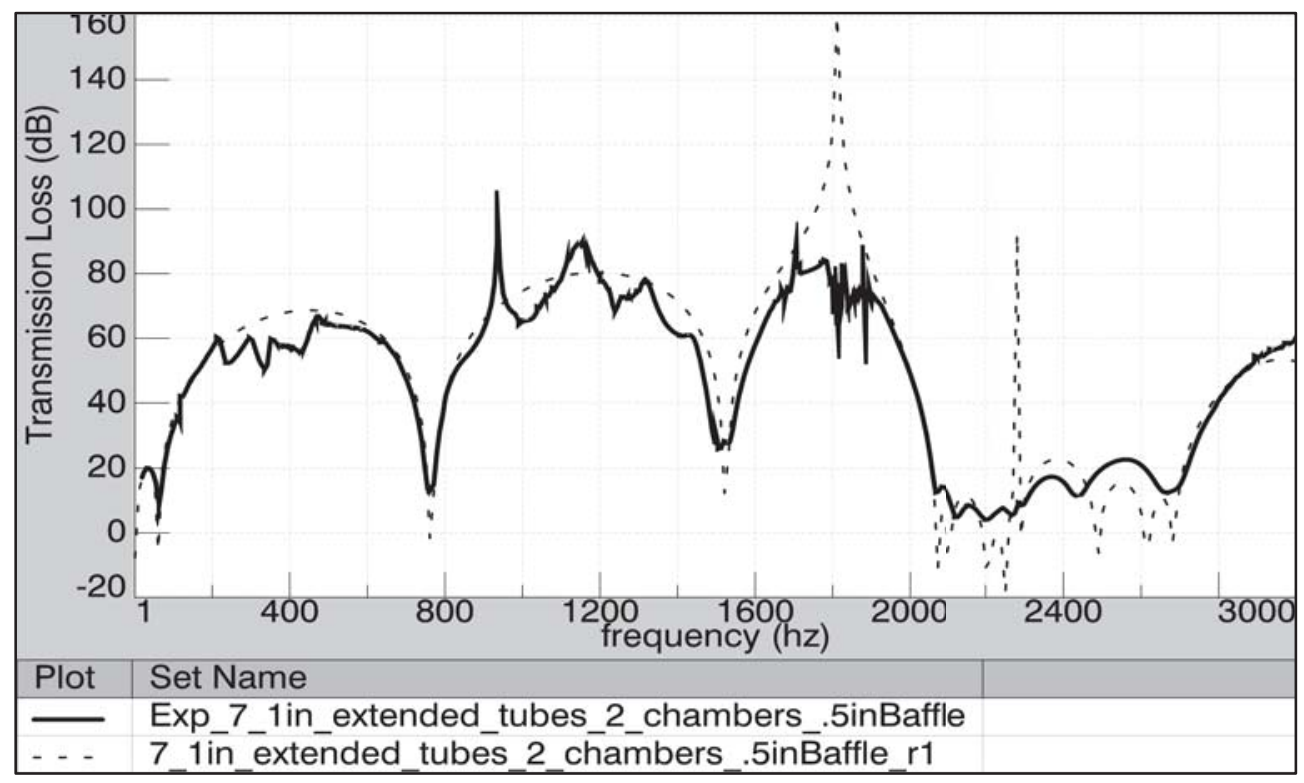

Figure 5.29: Case 7 correlation study - 1" extended tubes are added to the dual expansion chamber muffler 


\subsubsection{Correlation 7.1 - 1 " perforated tubing with 1 " extended tubes added to dual expansion chamber}

The next correlated model is the dual expansion chamber with 1" extended tubes and 1" perforated tubing. The results from the experimental and simulation testing are shown in Figure 5.30. Much like the previous case, the models correlate fairly well. Again, there are additional dynamics taking place in the experimental model. These dynamics seem to be slightly more pronounced in this scenario and the effects are assumed to be the result of the items discussed in the previous section.

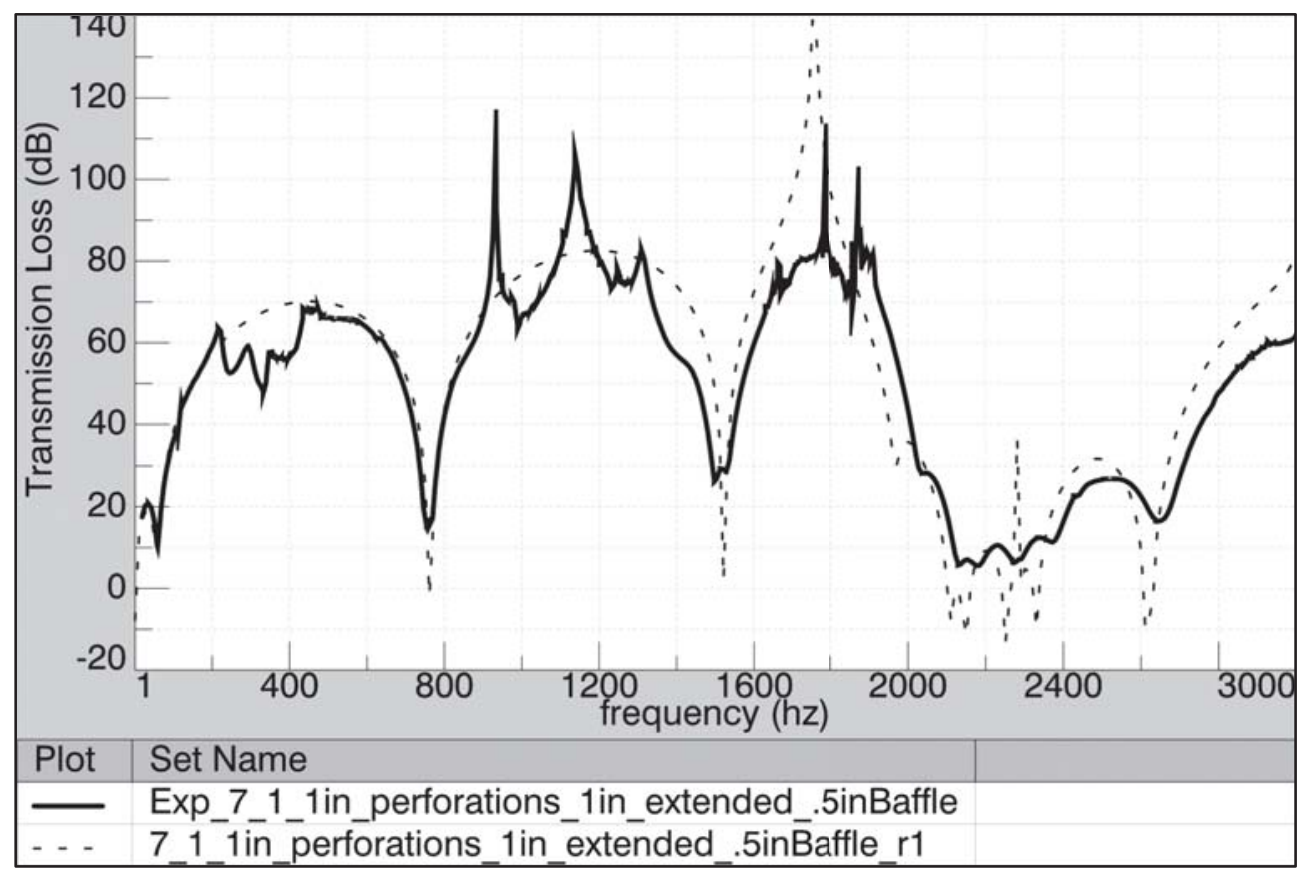

Figure 5.30: Case 7.1 correlation study - 1" perforated tubing with 1" extended tubes added to dual expansion chamber 


\subsubsection{Correlation 8 - Absorptive material with thicknesses of 0.25 " and 0.5 " added to dual expansion chamber with 1" extended tubes}

The last two correlated experimental models include absorptive material in the muffler with thicknesses of 0.25 " and 0.5 ". The results of the experimental and simulated models can be seen in Figure 5.31. For both thicknesses, the results show fairly good correlation in the low to mid frequencies. When the thickness is 0.25 ", the simulated model slightly under predicts. The simulated model under predicts by a fairly substantial amount for the 0.5 " model. As expected, the thicker the absorptive material the less attenuation in the low frequency range and the higher the attenuation in the mid to high frequency ranges. One of the difficulties with the absorptive material is determining the thickness of the material because when securing the material, it is often compressed a substantial amount.

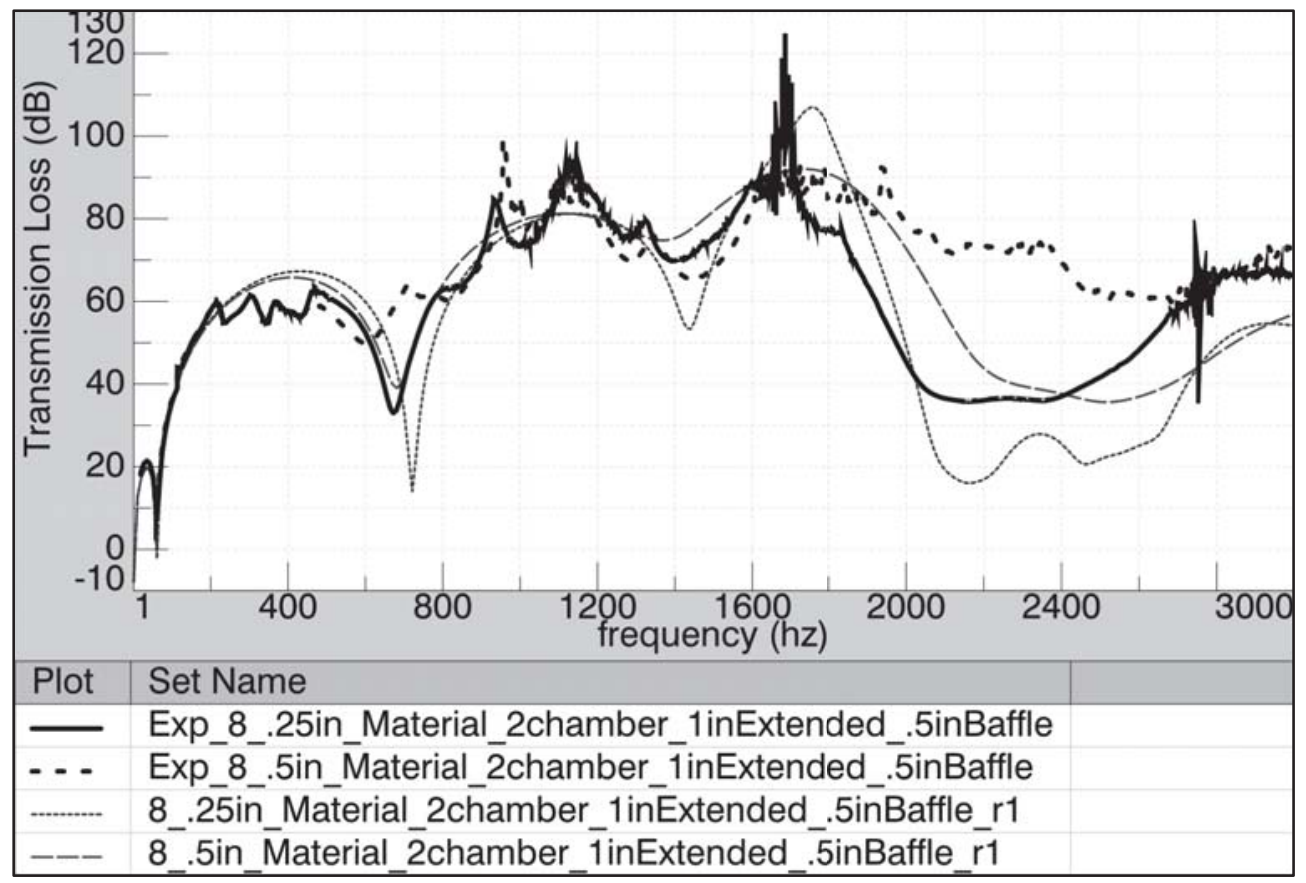

Figure 5.31: Case 8 correlation study - Absorptive material added to dual expansion chamber with 1 " extended tubes 


\subsection{Transmission Loss with mass flow rate}

As mentioned previously in Section 3.3.2, overcoming the flow noise in the microphones downstream of the muffler proved to be a daunting task. There were four different key methods used to overcome flow noise. The first method was to use the Stepped-Sine approach to measure FRFs which had a control strategy finely tuned for maximum output. Second, a new speaker was incorporated which produced more power. Third, a smaller diameter control muffler was selected, which provided slightly less attenuation. Finally, the source was applied as a vacuum which minimized flow noise.

The first muffler was a single expansion empty chamber which had dimensions shown in Figure 3.7, except the diameter is now 6". The steps describing how the test was conducted as well as how the control strategy was developed is discussed in Section 3.3.2. Due to the limitations in the equipment as previously discussed, the TL could only be accurately measured for flow speeds up to $35 \mathrm{~m} / \mathrm{s}(0.1 \mathrm{Ma})$.

The results for the first control muffler are displayed in Figure 5.32. As can be seen, when the flow speed was $0.05 \mathrm{Ma}$, the TL was not significantly affected although there may have been a slight drop. This was the same conclusion made for the simulation results for Case 9 when the diameter was 8 ". When the flow speed was increased to $0.1 \mathrm{Ma}$, it appears that the flow was affecting the measurement which did not allow for a real clean FRF. When looking at areas where there is not significant noise or fall out on TL, it may appear that the TL has

dropped by a miniscule amount. In order to conclude on the effects on mass flow, higher speeds need to be achieved. This was limited by the factors previously discussed in this test setup. It should be stated that the control strategy was the same for all three speeds. Since a fairly accurate TL was calculated for the no 


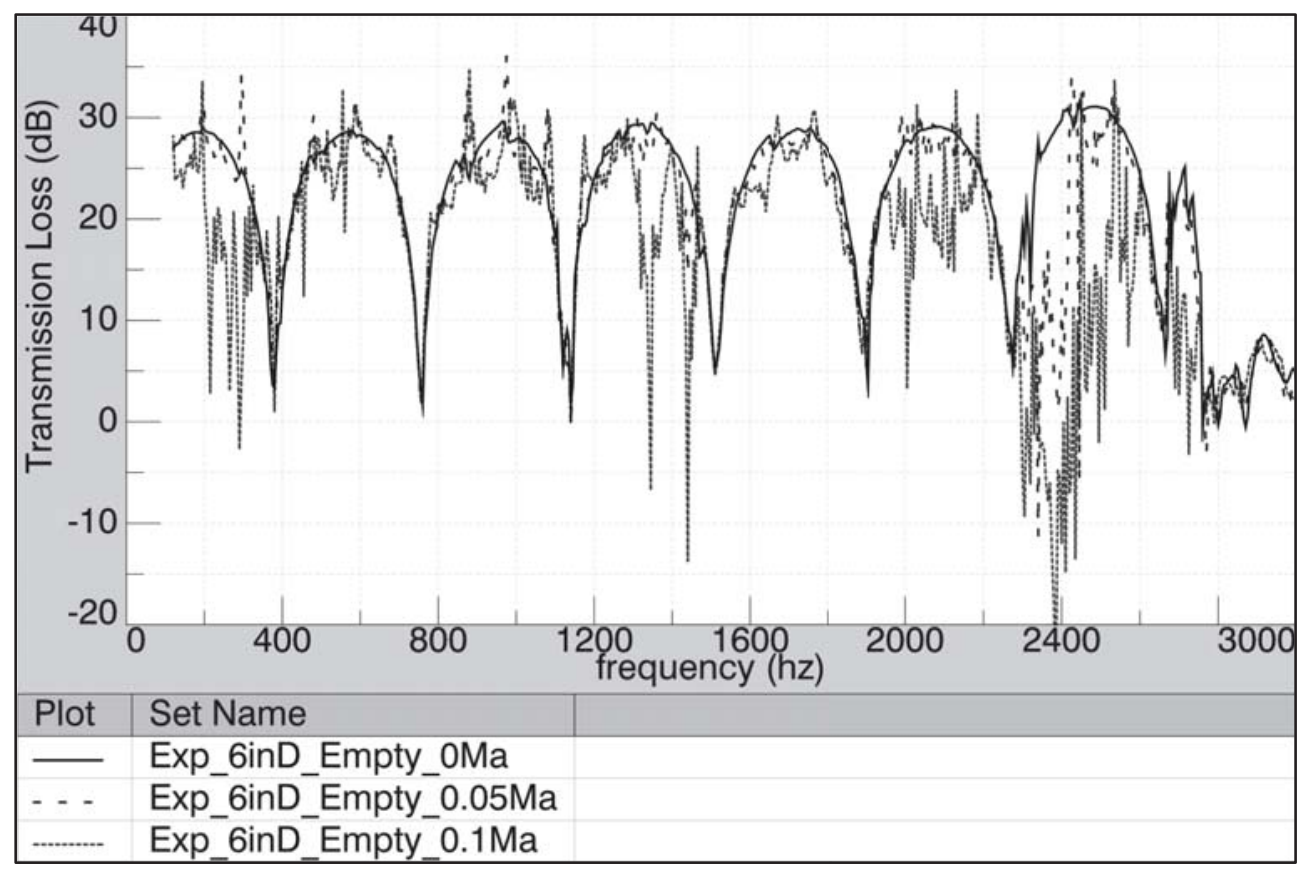

Figure 5.32: TL results for a 6" diameter empty muffler with mass flow.

speed configuration, it is fairly safe to say that exceeding the dynamic range of the microphones was not the leading cause for error in this testing.

The second muffler that was tested, which incorporated flow, had 1" perforated tubing installed on the previous muffler. A new control strategy needed to be developed because the dynamics of the muffler were changed. The resulting TL for the three flow speeds are shown in Figure 5.33. The perforated tubing caused the flow noise to be slightly reduced. The reduced flow noise lead to a more accurate TL measurement at all speeds. This topic will be discussed in the next section.

When a flow of $0.05 \mathrm{Ma}$ was applied to the muffler, there did not appear to be any substantial effect on TL. With a flow speed of 0.1 Ma there, again, appeared to be a drop out in TL at the very low and high frequencies. This is believed to be caused by excessive flow noise in the exhaust system which is negatively effecting the measurement. In the frequency span of 400 to $2200 \mathrm{~Hz}$, 
the flow does not seem to be affecting TL very much. There is too much noise in the measurements and higher velocities were not achievable. Therefore, a conclusion on the differences between these two mufflers cannot be made with $100 \%$ confidence. However, the experimentalist believes that overall the muffler with perforated tubing performs better than its counterpart when flow is implemented. It can be concluded that flow noise levels are lower when perforated tubing is used. This will be discussed in full in the next section.

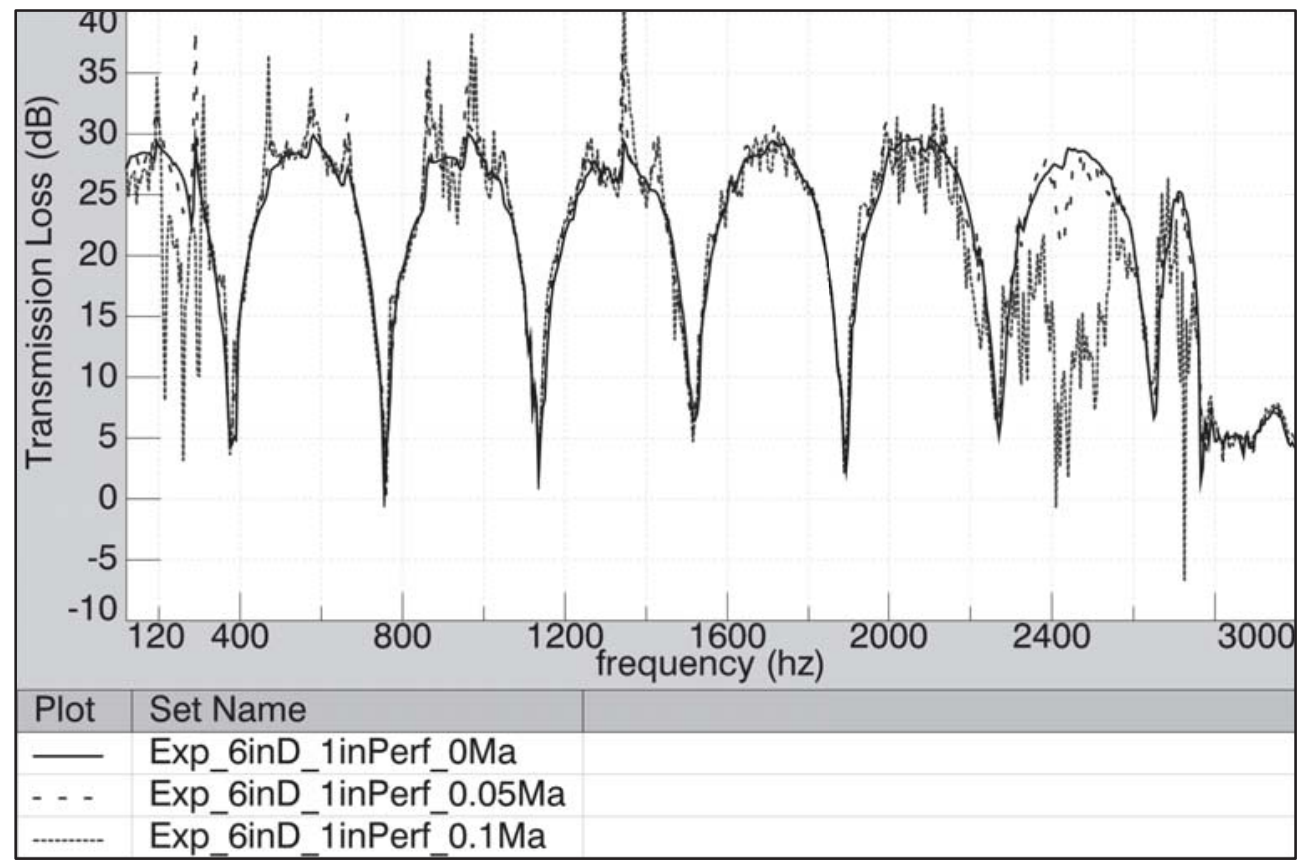

Figure 5.33: TL results for a 6" diameter muffler with 1" perforated tubing which incorporated mass flow. 


\subsection{Flow generated noise levels}

As discussed in Section 4.3, flow noise was measured at various rates for three muffler configurations inside of the tailpipe. The first two mufflers were used for the mass flow TL testing and the third muffler was the final experimental muffler tested for the correlation study. The final muffler consisted of a dual expansion chamber with 1" extended tubes and 1/2" absorptive material.

The source that was creating the flow in this test setup was a $2.5 \mathrm{Hp}$ shop vacuum. The vacuum had the ability to be placed downstream of the muffler (used as a vacuum) or placed upstream of the muffler (used as a blower). Because of the versatility, both configurations were tested to see the effects. For the three mufflers tested, the flow noise was measured at speeds of $17.5 \mathrm{~m} / \mathrm{s}, 35 \mathrm{~m} / \mathrm{s}$, and $47 \mathrm{~m} / \mathrm{s}$ (maximum capacity of the shop vacuum) for both source configurations. The resulting linear spectrums for both source configurations under full load conditions can be seen in Figure 5.34 and Figure 5.35. The lowest resulting flow noise method (vacuum) was used for mass flow TL testing.

The results show that when the source was upstream of the muffler, the flow noise was considerably higher. This may have been caused by a combination of two reasons. The first is that the flow was more turbulent which would increase the noise generation efficiency of the exhaust system (21). The second is the source noise was convected downstream; this wouldn't have been the case if the source was applied downstream of the muffler. The other finding is that overall the addition of the perforated tubing decreased the flow generated noise, however, there was particular speeds where there was little to no improvement. This was the case in Figure 5.35 when the source was used as a vacuum. One of the reasoning's for this is that for the flow speeds applied, monopole sources dominated the system (21). As monopole sources are the lowest order, there is no way to lower 
the source type. It is believed that to fully see the effects of the flow, as well as the effects of the perforated properties, higher order sources (dipole/quadrupole sources) need to be present in the exhaust system.

The next test performed was a run-up for the baseline muffler when the source was used as a vacuum. This was to confirm that the sources present in the exhaust system for this model setup was monopole sources. The results from the run-up are shown in Figure 5.36. Based on the work presented in Section 2.5.3 and using Equation 2-44 through Equation 2-46, the source type for this configuration was calculated to be 1.13. Based on the finding, it can be concluded that mainly monopole sources are present for these speeds. From the works of Wiemeler et al., the results showed that typically monopole sources were present in the range of 0$0.1 \mathrm{Ma}$, dipole sources were dominate in the range of 0.1-0.25, and quadrupole sources were dominate after $0.25 \mathrm{Ma}$ (21). 


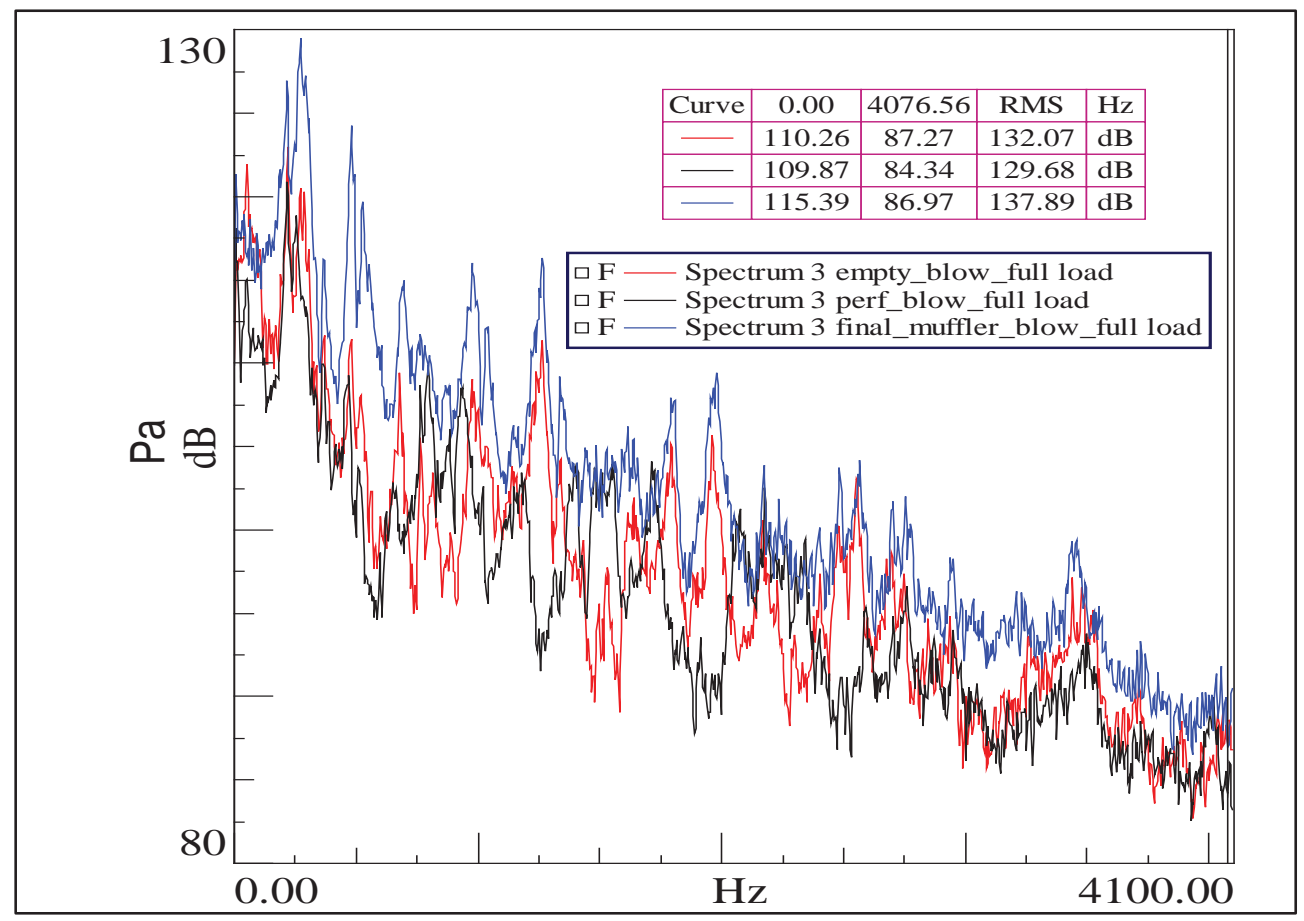

Figure 5.34: Flow noise generated inside of the mufflers when the source is used as a blower and applied a velocity of $47 \mathrm{~m} / \mathrm{s}$ to the inlet.

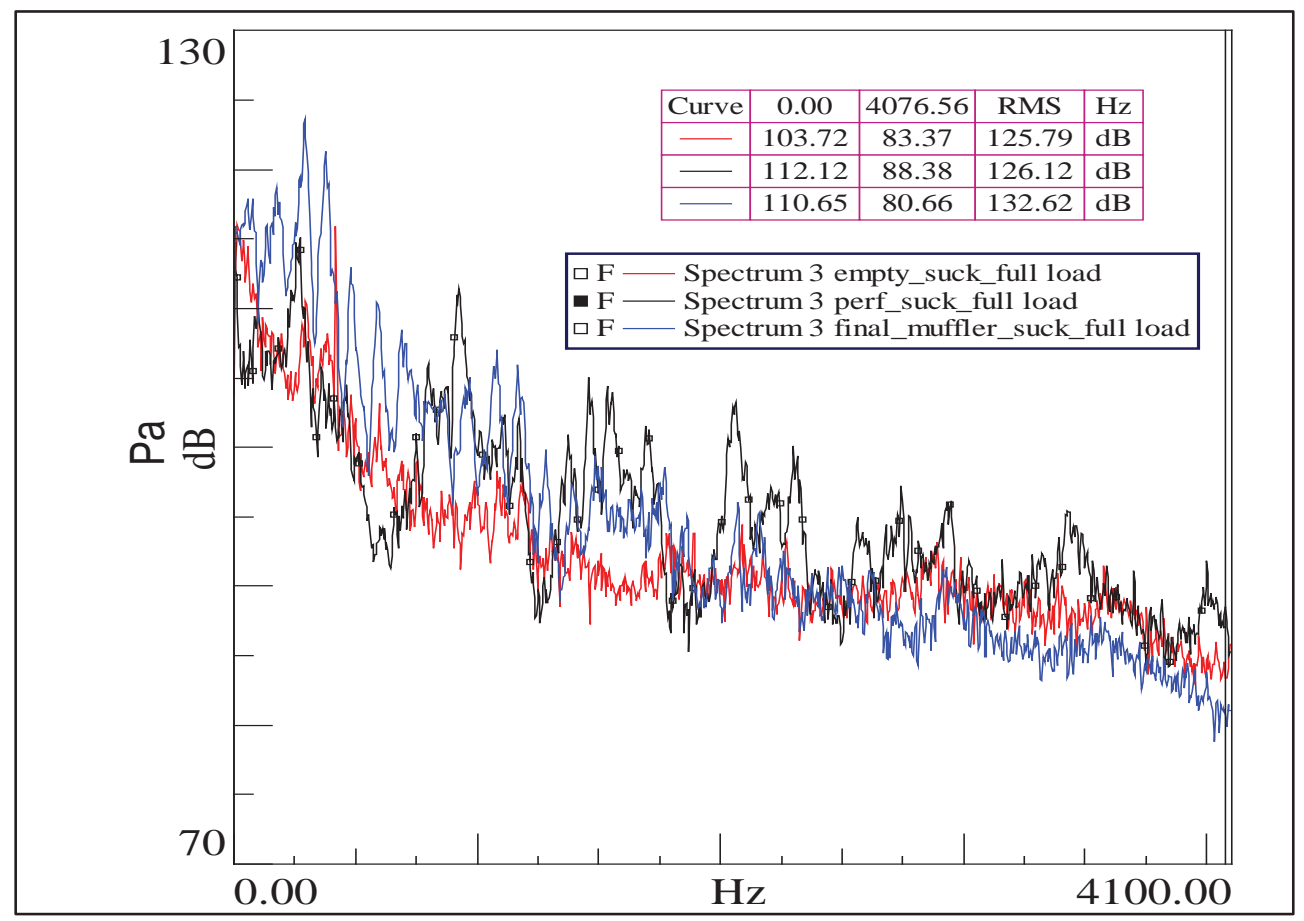

Figure 5.35: Flow noise generated inside of the mufflers when the source is used as a blower and applied a velocity of $47 \mathrm{~m} / \mathrm{s}$ to the inlet. 


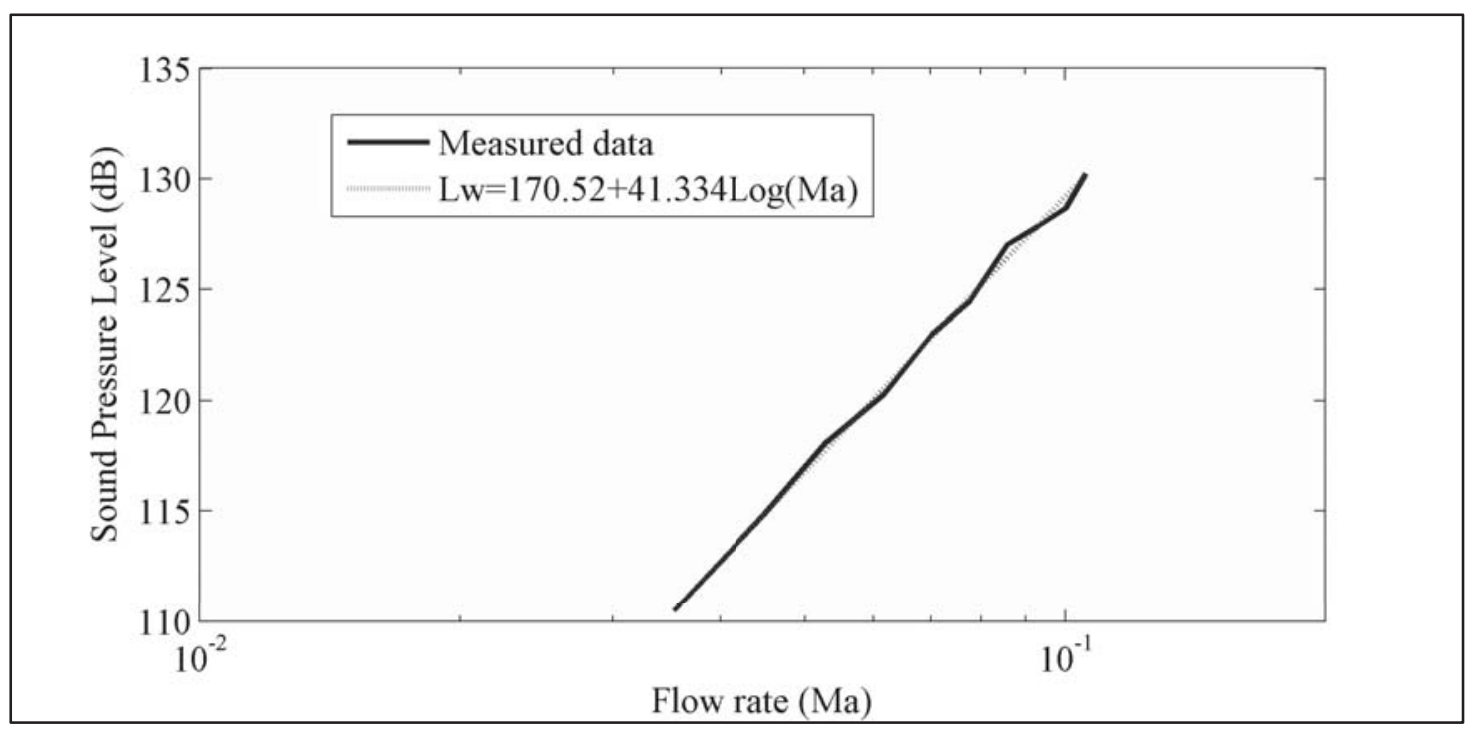

Figure 5.36: The overall sound pressure level as a function of the flow speed for the 6" Diameter empty muffler. 


\section{Chapter 6. Future work}

This thesis covered many topics in current research of acoustic performance prediction of exhaust systems but there are still several areas which can be further researched. Some of the topics where future research is heavily needed will be discussed in this chapter.

\subsection{Source modeling}

If sound pressure levels are to be predicted in the free field than the source properties need to be known. The experimental methods for determining source impedance on a muffler can be very difficult, time consuming, and costly. As a result, there has been a lot of research recently in using simulation tools such as GT-Power to determine acoustic source properties. Research in these areas and correlation to experimental techniques would be very beneficial.

\subsubsection{Predicting sound pressure levels}

After determining the source properties, either by experimental or simulation techniques, the results can be applied a model as discussed in Section 4.2.6 using FEM tools. Other techniques to predict SPLs that could be used are when the source properties are implemented in GT-Power and the 4-pole parameters, calculated using FEM/BEM, are also incorporated into the 1-D model. These methods could be correlated to experimental data and conclusions can be made on the accuracy of the various techniques. 


\subsection{Incorporating temperature effects in experimental setup}

One of the difficult things to do in a simulation model is to apply the correct temperature profile. As discussed in this thesis, the accuracy of the temperature profile is crucial in determining sound pressure levels in the free field for a running engine. By applying temperature effects to the test setup which already includes air flow, the actual running condition of the engine can be approximated fairly well. The difficulty with including the temperature effects would be the survivability of the equipment. The current test setup would need to be modified so the speaker and shop vacuum do not see high temperature levels. Also, pressure taps would need to replace microphones and adapters would need to be fabricated.

\subsection{Perforated tubing}

Perforated tubing is often used to stabilize the flow inside of the exhaust system and to maximize the expansion of the acoustical waves. Now that the flow bench is developed, the effects of perforated tubing properties can be investigated more rigorously. This can include how much flow noise is generated and how the TL of the exhaust system is effected for various hole patterns, spacing, diameter, etc...

\subsection{Increasing capability of TL measurement with flow}

As described in Section 3.3.2, using the implemented Stepped-Sine approach had its limitations. This method can be improved by using a few techniques. The first improvement would be to have the control channel in the 
Stepped-Sine approach be an accelerometer placed on the speaker. During this research, the control channel was one of the microphones in the test tubes.

Developing the control algorithm was a difficult challenge as the flow noise would degrade the quality of the measurement. By using the accelerometer, the flow noise would relatively unaffect the transducer measurement. This would lead to better control of the Stepped-Sine algorithm.

Also, using different FRF estimators, such as $H_{r}$ or $H_{c}$, could be used. These estimators have the ability to extract the necessary information from the unwanted noise. In this case, the FRF at the microphone locations created from the speaker could be separated from the FRF created by the flow noise. As a result, the TL could be measured for drastically higher flow speeds. There also would be no flow noise deteriorating the TL measurement, which caused the decrease in TL quality in this thesis. 


\section{Chapter 7. Conclusion}

The works in this thesis proved the validity of simulation techniques to adequately model an exhaust system and to predict the Transmission Loss of a muffler. The fundamental elements used in exhaust systems were investigated and their effects on performance were determined through simulation and experimental techniques. Other areas on how to accurately model perforated tubing, absorptive material, and temperature profiles were discussed and implemented. From the results achieved, a designer can use the findings in this thesis to optimize the design of a muffler.

Perforated tubing behaves as a dissipative element. When flow grazes passed the perforated holes, a portion of the acoustical energy is converted to heat (2). The energy conversion is typically more efficient in the mid to high frequency range which usually causes a slight increase in TL. The primary use of perforated tubing, however, is to combat mass flow effects. As flow exits an extended tube, there frequently is a significant amount of turbulence. The perforated tubing helps to lower the source type which will greatly reduce the flow noise generated, as described in the findings of Wiemeler et al.21). Perforated tubing can also be beneficial to maximize available expansion space. If the relationship of the spacing between the inlet and outlet tube is relatively small compared to the expansion tube diameter, the acoustical waves do not fully expand.

Absorptive materials are another form of a dissipative element. As presented in the findings, the material levels out the peaks and toughs of the TL as well as increase the amplitude in the mid to high frequency ranges. The sound quality of the exhaust system is greatly improved for a leveled out TL. This is because the attenuation provided by the exhaust system will have a fairly linear behavior with increased operating speeds. The absorptive materials negatively 
affect the TL in the low frequency range because the materials reduce the allowable expansion of the acoustic waves. Therefore, there is always a finite amount of material that is ideal for every scenario.

The temperature gradients seen in an exhaust system cause the attenuation properties to behave differently. An increased temperature influences both the speed of sound and the density of the medium. These two properties, particularly speed of sound, greatly control the TL of the muffler. In general, the TL is shifted to the right which is governed by Equation 2-41. This equation is applicable for a constant elevated temperature throughout the system. This clearly wouldn't be the case in operation where the temperature would vary depending on the location in the chamber. With a varying temperature profile, there is a distinct shift in TL but there are also erratic amplitude effects as well. These amplitude effects cannot be predicted without the use of FEM or BEM.

When flow is introduced into an exhaust system, the overall TL of the muffler can be altered. The flow strengthens the progressive wave while weakening the reflective wave (2). This effect, in general, causes an overall decrease in amplitude of the TL with increasing mass flow. The influence of flow only appears significant at higher speeds, as is the case for the simulation results. The author could not experimentally measure very fast flow speeds (above .25 $\mathrm{Ma}$ ), therefore, the air flow did not seem to greatly influence the TL of the control mufflers.

When exhaust gas flows through the muffler system, there is a great deal of noise generated from the turbulent flow. The noise generated is classified as "flow noise" and is dependent on the source type(s) present in the exhaust. For flow speeds usually seen in an exhaust system, dipole sources dominate (21). However, there may be locations where quadrupole sources exist, particularly around tight bends and sudden expansions. At these locations a great deal of flow noise is 
generated, often dominating the sound of the pulsating pressure wave. Therefore, it is crucial to locate these locations and apply appropriate corrections to the model. The most appropriate method for this is through the use of CFD to find locations of high air flow (above $0.3 \mathrm{Ma}$ ).

Experimental techniques for determining the Transmission Loss of a muffler were presented. The most effective method, two source-location, was practiced which resulted in accurate TL measurements. The limitations of the experimental test step were discussed which included the dynamic range of the microphones, speaker power output, and speaker frequency range produced.

The process of modeling the source was presented in detail. The theory of the source modeling, as well as the correct implementation, was laid out within this thesis. As a result, the reader now has the tools to sufficiently predict the overall performance of the exhaust system when the source is implemented into the model. Although no data was presented in this thesis on the accuracy of these models, the author has practiced these techniques on a confidential project and achieved fairly high correlation.

Secondary effects on muffler performance, such as flow effects on TL and flow noise generated, were presented. The TL of the muffler could not be measured with flow speeds as high as the author had hoped for. However, with the necessary upgrades in the equipment discussed, higher speeds are feasible. The flow bench in place can also be used to determine how much noise is generated by the flow, which was practiced in this thesis. The results can be used to determine the source properties in the flow which can be very useful for design optimization and comparison purposes. 


\section{References}

1. Shah S, Kuppili S, Hatti K, Thombare T. A Practical Approach towards Muffler Design. SAE International. 2010-32-0021.

2. Munjal ML. Acoustics of Ducts and Mufflers with Application to Exhaust and Ventilation System Design. $1^{\text {st }}$ ed. New York(NY) : John Wiley \& Sons, Inc.; 1987.

3. Mohanty AR, Pattnaik SP. An Optimal Design Methodology for a Family of Perforated Mufflers. SAE International. 2005-26-053.

4. Ferguson C, Kilpatrick A. Internal Combustion Engines. $1^{\text {st }}$ ed. New York(NY): John Wiley \& Sons, Inc.; 2001.

5. Ji Z. Acoustic Attenuation Performance of a Multi-chamber Muffler with Selective Sound-absorbing Material Placement. SAE International. 200701-2202.

6. Keske JD. Investigation of a Semi-active Muffler System with Implementation on a Snowmobile [PhD Dissertation]. Houghton (MI). Michigan Technological University. 2009.

7. Selamet A, Denia FD, Besa AJ. Acoustic Behavior of Circular DualChamber Mufflers. Journal of Sound and Vibration. 2003; 265: 967-985.

8. Selamet A, Ji ZL. Acoustic Attenuation Performance of Circular Expansion Chambers with Extended Inlet/Outlet. Journal of Sound and Vibration. 1999; 223(2): 197-212. 
9. Selamet A, Ji ZL. Acoustic Attenuation Performance of Circular Expansion Chambers with Offset Inlet/Outlet: I. Analytical Approach. Journal of Sound and Vibration. 1998; 213(4): 601-617.

10. Selamet A, Ji ZL. Acoustic Attenuation Performance of Circular Expansion Chambers with Offset Inlet/Outlet: II. Comparison with Experimental and Computational Studies. Journal of Sound and Vibration. 1998; 213(4): 601617.

11. Selamet A, Radavich PM. The Effect of Length on the Acoustic Attenuation Performance of Concentric Expansion Chambers: An Analytical, Computational and Experimental Investigation. Journal of Sound and Vibration. 1997; 201(4): 407-426.

12. Ji Z, Su S, Liu C. Acoustic Attenuation Performance Analysis of Threepass Perforated Tube Muffler with End-resonator. SAE International. 200801-0894.

13. Siano D, Bozza F, Auriemma F. Pros and Cons of Using Different Numerical Techniques for Transmission Loss Evaluation of a Small Engine Muffler. SAE International. 2010-32-0028.

14. Sullivan JW, Crocker MJ. Analysis of concentric-tube resonator having unpartitioned cavities. Journal of the Acoustical Society of America. 1978;64: 207-215.

15. Anderson KS. Analysis of Exhaust Elements Using the Transfer Matrix Method. SAE International. 2010-01-1426.

16. Sen S. Prediction of Flow and Acoustical Performance of an Automotive Exhaust System using 3-D CFD. SAE International. 2011-01-1068. 
17. Fairbrother R, Varhos E. Acoustic Simulation of an Automotive Muffler with Perforated Baffles and Pipes. SAE International. 2007-01-2206.

18. Yadav PS, Gaikwad AA, Kunde SA, Karanth NV. Prediction of Muffler Radiated Noise for a Diesel Engine. SAE International. 2011-26-0065.

19. Callow GD, Peat KS. Insertion Loss of Engine Inflow and Exhaust Silencers. I Mech. E. 1988;19:88.

20. Fairbrother R, Boden H, Glav R. Linear Acoustic Exhaust System Simulation Using Source Data from Non Linear Simulation. SAE International. 2005-01-2358.

21. Zheng S, Kang ZX, Lian XM. Acoustic Matching Simulation of Muffler with Hybrid Approach. SAE International. 2011-01-1516.

22. Wiemeler D, Jauer A, Brand JF. Flow Noise Level Prediciton Methods of Exhaust System Tailpipe Noise. SAE International. 2008-01-0404.

23. Lord HW, Gatley WS, Evensen HA. Noise Control for Engineers. $1^{\text {st }}$ ed. Malabar(FL): Krieger Publishing Company; 1987.

24. LMS Virtual.Lab. LMS Virtual.Lab FEM Acoustic - Perforated Muffler. LMS Virtual.Lab Rev6B SL1 VL Acoustic Training - Acoustic FEM tutorial. 2007.

25. Newton - Ask a Scientist! DOE Office of Science [Internet]. Argonne (IL): Newton and ask scientist. Air Density Formula; 2011[updated 2011 Sept, cited 2011 Oct 2]. Available from: http://www.newton.dep.anl.gov/askasci/wea00/wea00270.htm 
26. Montenegro G, Della TA, Onorati A, Fairbrother R, Dolinar A. Development and Application of 3-D Generic Cells to the Acoustic Modeling of Exhaust Systems. SAE International. 2011-01-1526.

27. Cheng CYR, Wu TW, Exhaust Muffler Design and Analysis Using a Boundary Element Method Based Computer Program. SAE International. 1999-01-1661.

28. Tao Z, Seybert AF. A Review of Current Techniques for Measuring Muffler Transmission Loss. SAE International. 2003-01-1653.

29. Kim MH. Three-Dimensional Numerical Study on the Pulsating Flow inside Automotive Muffler with Complicated Flow Path. SAE International. 2001-01-0944.

30. LMS Virtual.Lab. LMS Virtual.Lab FEM Acoustic - Muffler TL Analysis with Mean Flow Effect. LMS Virtual.Lab Rev8B VL Acoustic Training Acoustic FEM tutorial. 2008.

31. HyperPhysics [Internet]. Atlanta(GA): Speed of Sound Formula; 2009 [cited 12 Oct. 2011]. Available from: http://hyperphysics.phyastr.gsu.edu/hbase/sound/souspe3.html 


\section{Appendices}

The following appendices supplement the information presented within this thesis.

\subsection{Appendix A: Additional Figures from FEM Study}

A parametric study was conducted on how modifying element properties affected the Transmission Loss. The results from these findings were presented in Chapter 5. The following figures are used to describe the effects of adding additional elements to the exhaust system, rather than the results from modifying their properties.

In Figure A.1, the TLs were calculated using the FEM for various mufflers. The initial "baseline" muffler was the empty cylindrical expansion chamber with dimensions given in Figure 3.7. The second muffler had 1" extended tubes added to the empty chamber. The third muffler had 1" extended tubes and 1" perforated tubing added to the empty chamber. The addition of the extended tube caused a gradual increase in TL to a maximum difference of $+9.5 \mathrm{~dB}$. As this was a gradual increase, the low frequency TL was relatively unaffected. The length of the extended tube does not cause an acoustical resonance in the frequency range of interest. The addition of the perforated tubing had a similar effect on TL as the extended tube did. There was a maximum difference of $+14 \mathrm{~dB}$ compared to the empty "baseline" chamber. 


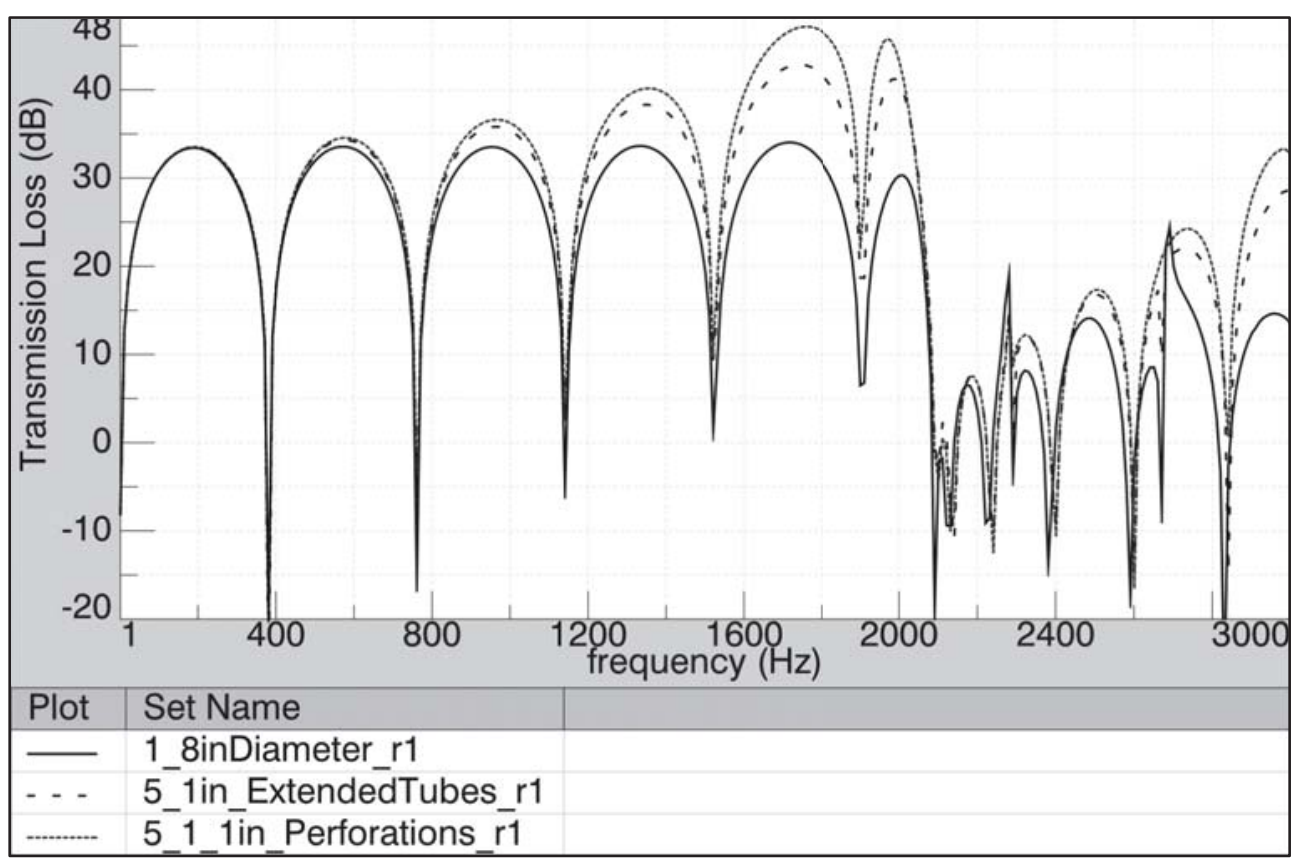

Figure A.1: FEM results for a few single expansion chamber mufflers.

In Figure A.2, the TL was measured experimentally for the same three mufflers used in the previous simulation models shown in Figure A.1. The experimental results followed the same trend pattern seen in simulation model however there was some differences. The simulation model under predicted the effects of the extended tubes. The maximum difference in amplitude was measured to be $+12 \mathrm{~dB}$ between the experimental models with and without extended tubes. The perforated tubing appeared to have a slightly negative effect (up to $-.5 \mathrm{~dB}$ ) when added to the extended tube model. However, the overall amplitude of the TL was nearly identical between the simulation and experimental models when perforated tubing was added. 


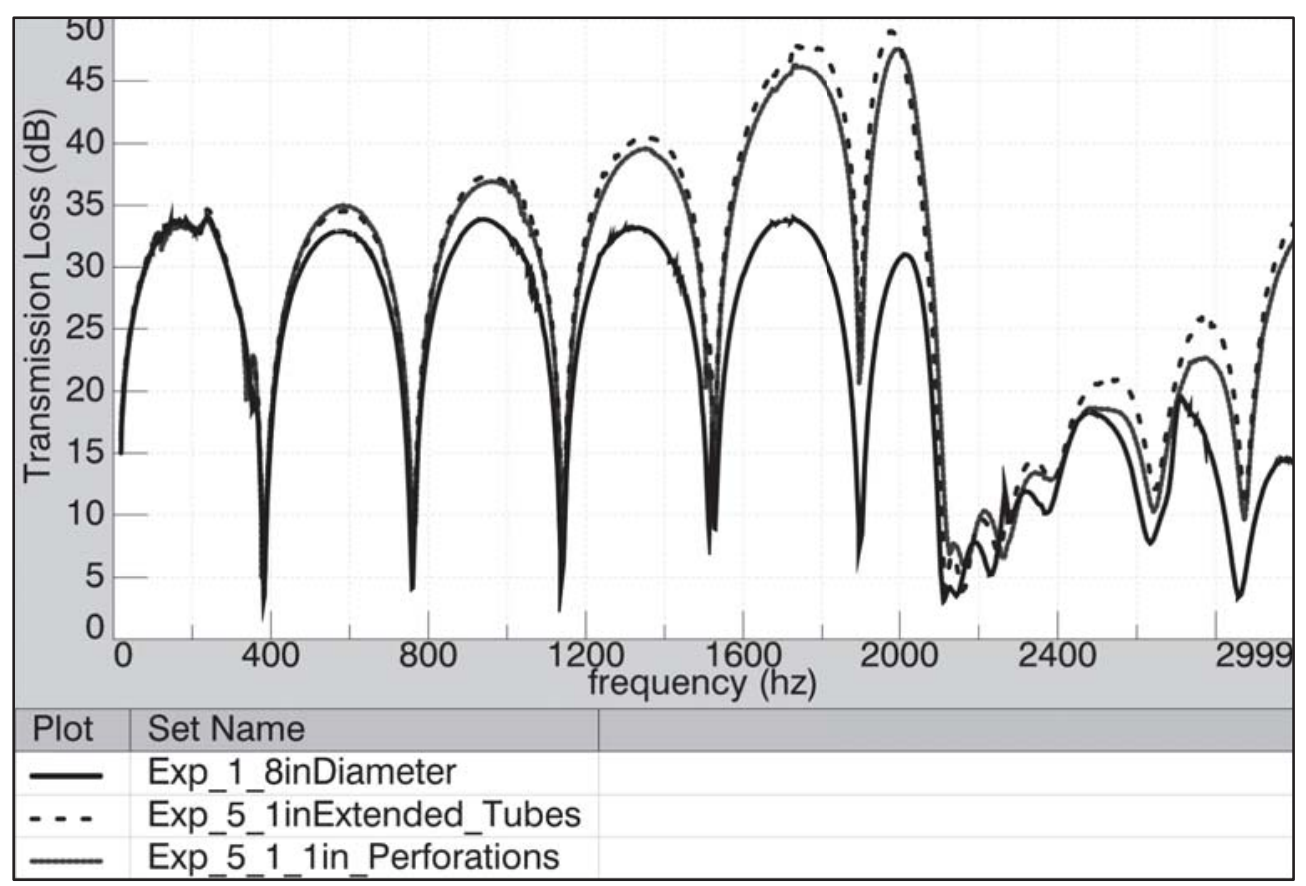

Figure A.2: Experimental results for a few single expansion chamber mufflers.

In Figure A.3, the TLs were calculated using the FEM for various mufflers. The initial "baseline" muffler was the empty cylindrical dual expansion chamber with a center baffle 0.5 " thick. The second muffler had 1 " extended tubes added to the empty chamber. The third muffler had 1" extended tubes and 1" perforated tubing added to the empty chamber. The final two mufflers had 0.25 " and 0.5 " of absorptive material added to the third muffler configuration, respectively. The addition of the extended tube shifted the first major dome in the TL to the left considerably. This is a major benefit as typically the first major firing frequency is relatively low in the frequency spectrum. Overall the amplitude of the TL was increased by roughly $10 \mathrm{~dB}$ with the addition of the extended tubing. The perforated tubing raised the TL by an additional $1.5 \mathrm{~dB}$ across the entire frequency spectrum. The addition of the absorptive material leveled out the peaks and troughs in the model. There was an increase in TL at the mid and high frequency spectrum. The low frequency spectrum was negatively affected as the expansion area was reduced by the material. 


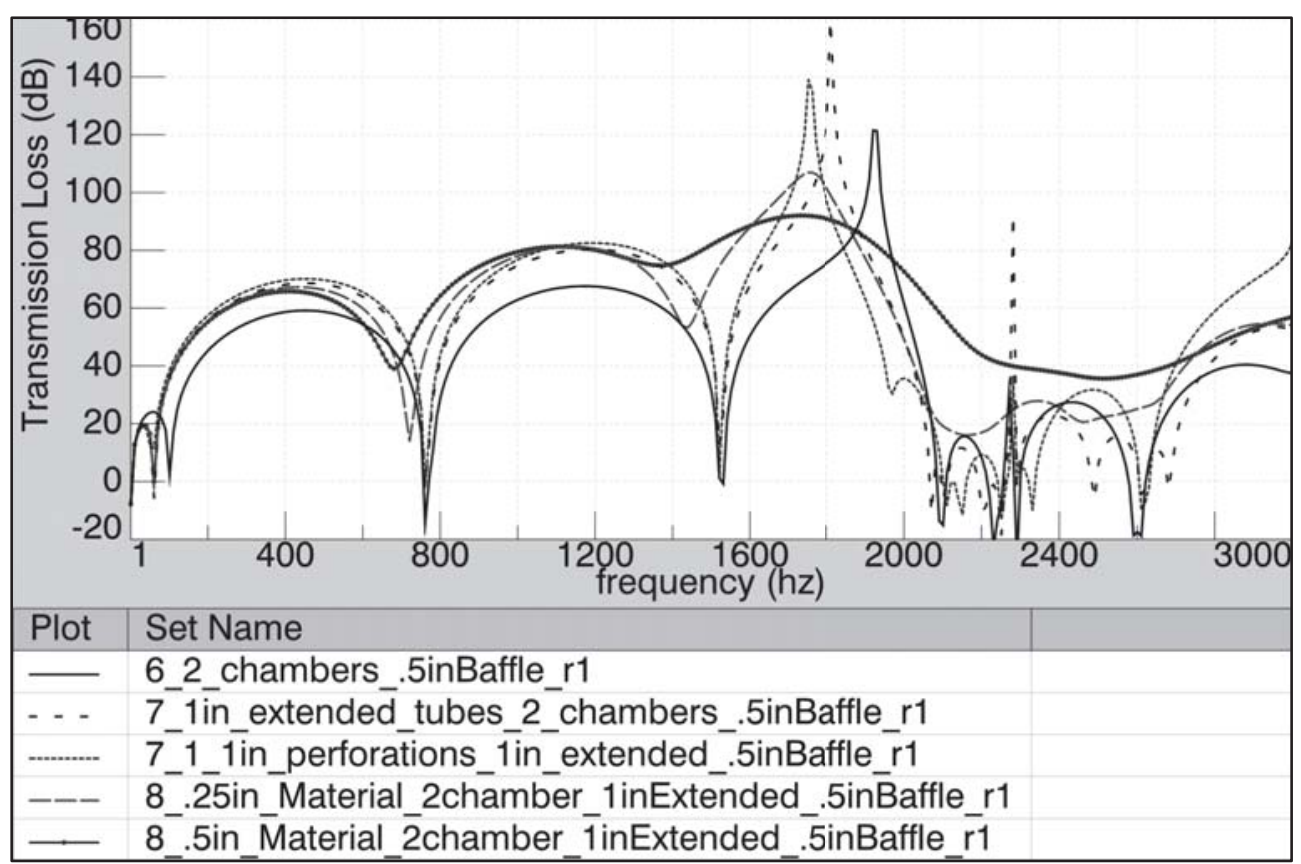

Figure A.3: FEM results for a few dual expansion chamber mufflers.

In Figure A.4, the TL was measured experimentally for the same five mufflers used in the previous simulation models shown in Figure A-3. The experimental results followed the same trend pattern seen in simulation model however there appears to be more dynamics taking place in the models. Exceeding the dynamic ranges of the microphones became a major issue as the TL is in access of $80 \mathrm{~dB}$ at many locations. These effects were discussed in detail within the thesis. 


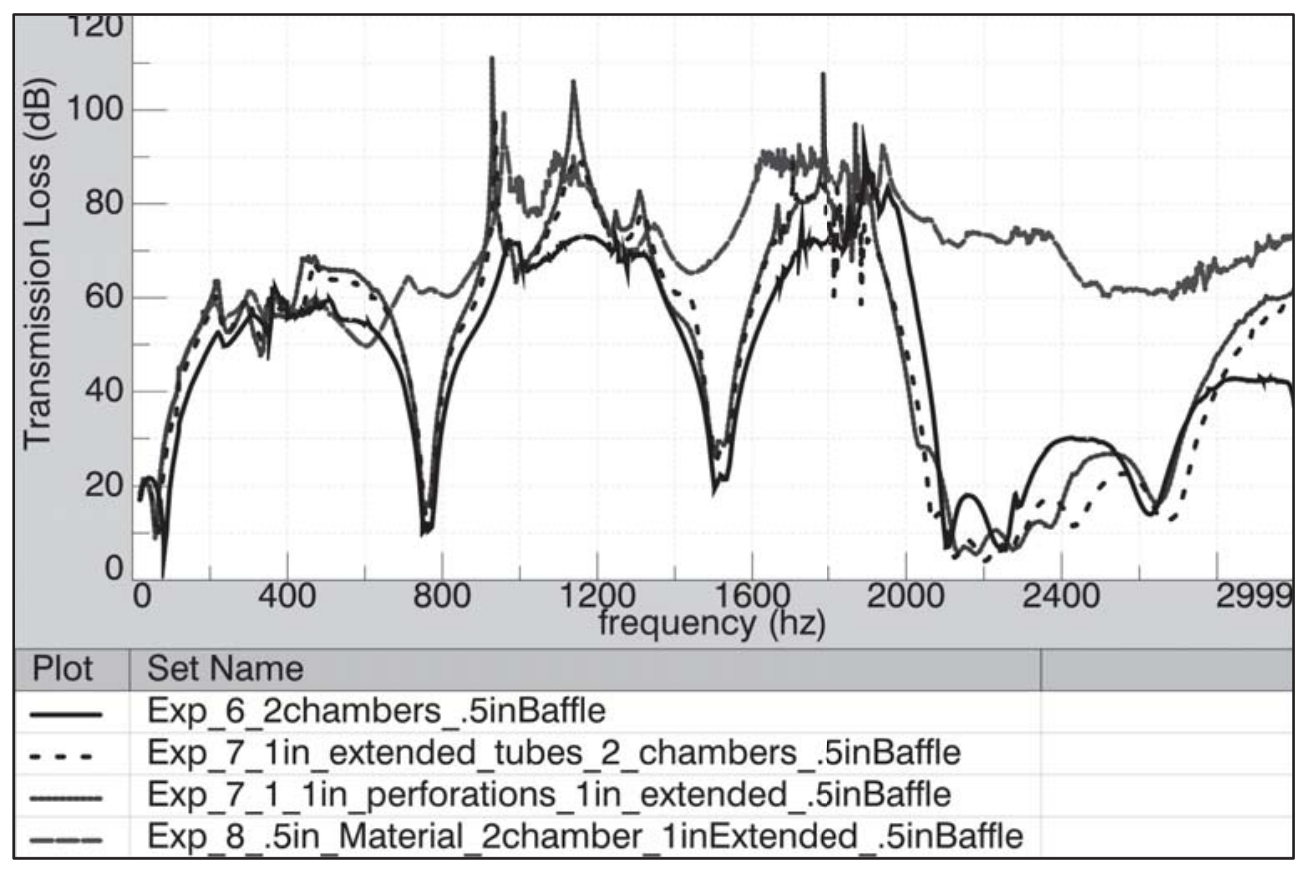

Figure A.4: FEM results for a few dual expansion chamber mufflers. 


\subsection{Appendix B: Perforated Tubing MatLab Script}

Below is the MatLab code used to determine the impedance properties of the perforated tubing.

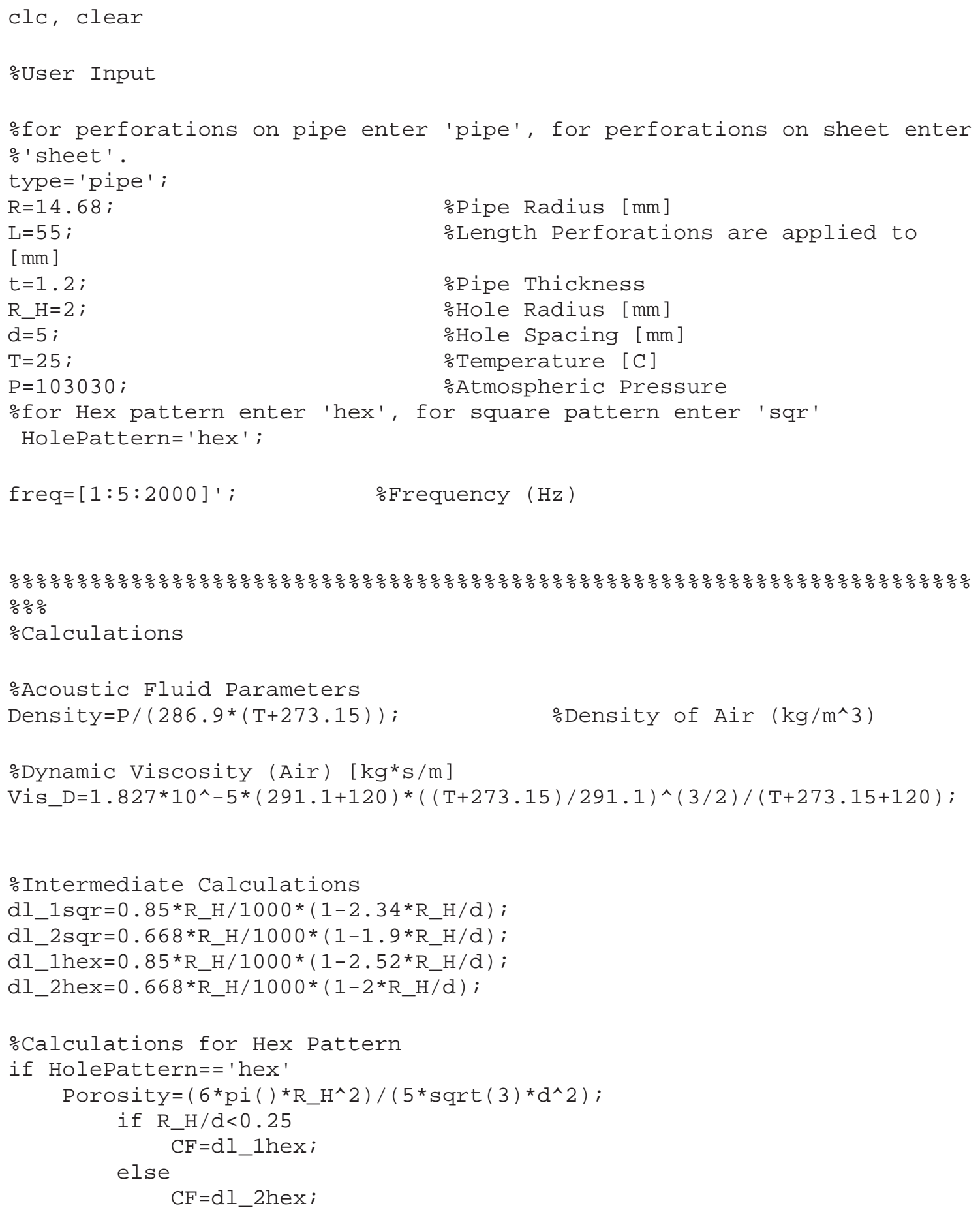




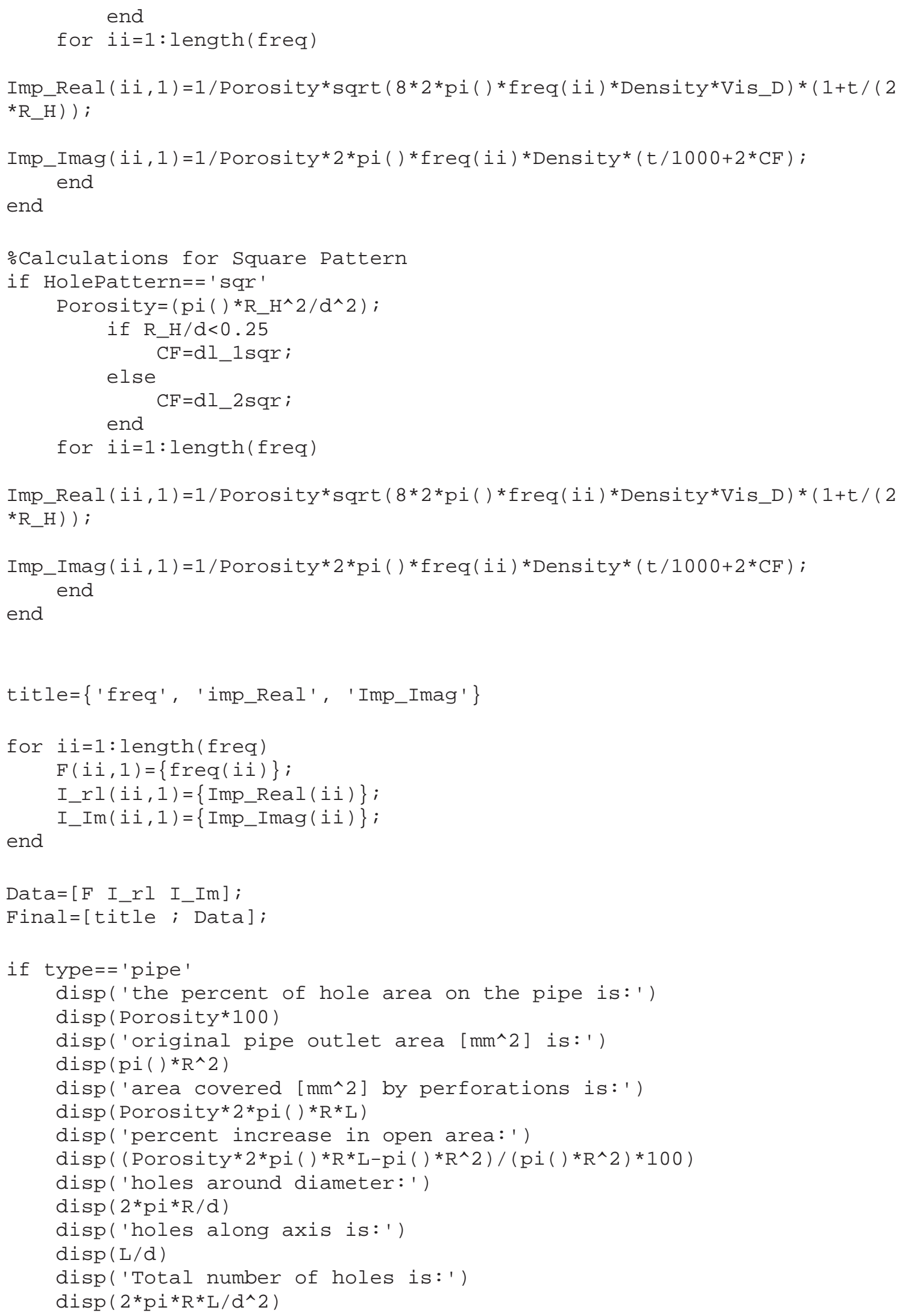




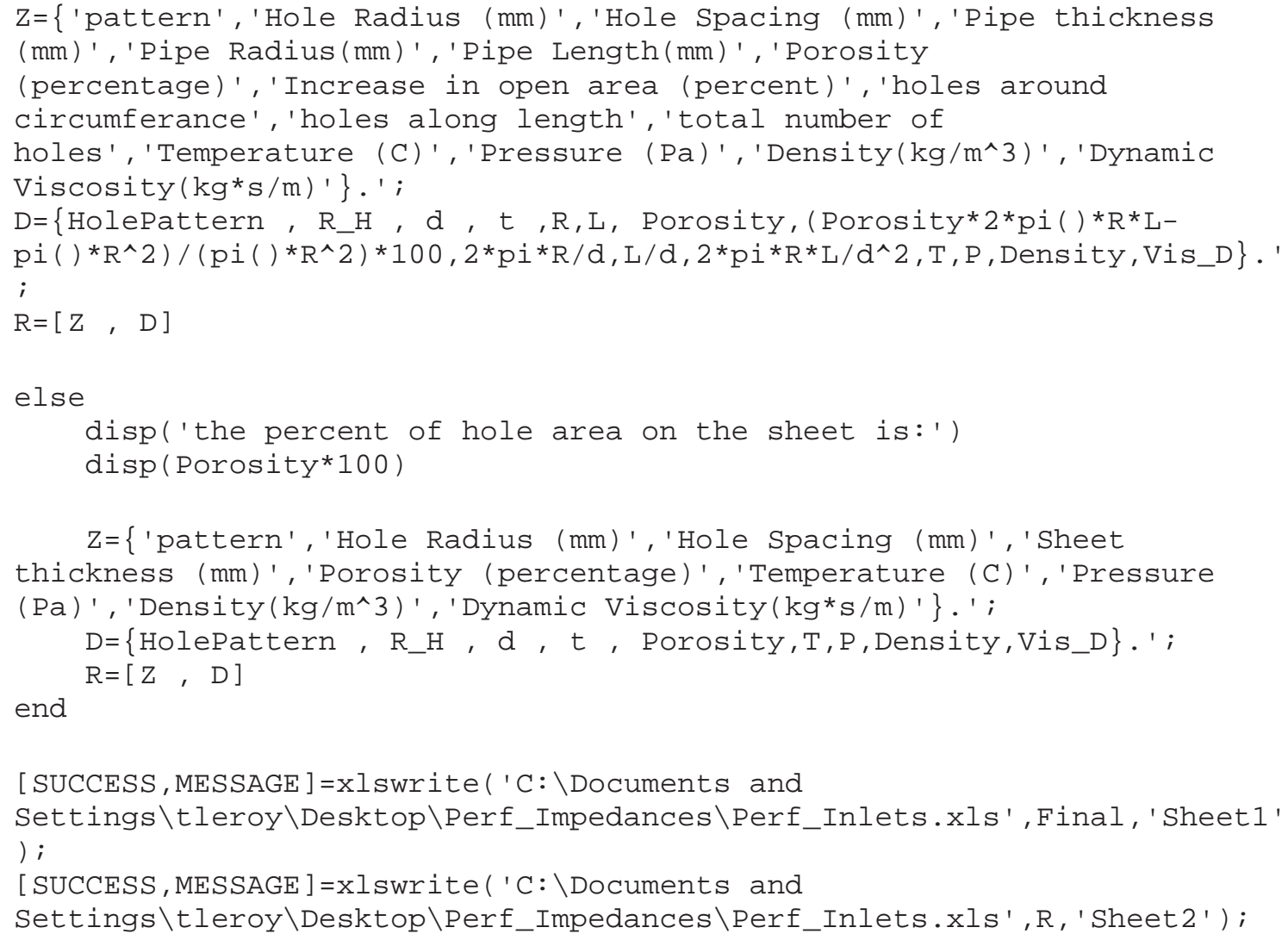

